\title{
SPECTRAL AND HODGE THEORY OF 'WITT' INCOMPLETE CUSP EDGE SPACES
}

\author{
JESSE GELL-REDMAN AND JAN SWOBODA
}

\begin{abstract}
Incomplete cusp edges model the behavior of the Weil-Petersson metric on the compactified Riemann moduli space near the interior of a divisor. Assuming such a space is Witt, we construct a fundamental solution to the heat equation, and using a precise description of its asymptotic behavior at the singular set, we prove that the Hodge-Laplacian on differential forms is essentially self-adjoint, with discrete spectrum satisfying Weyl asymptotics. We go on to prove bounds on the growth of $L^{2}$-harmonic forms at the singular set and to prove a Hodge theorem, namely that the space of $L^{2}$ harmonic forms is naturally isomorphic to the middle-perversity intersection cohomology. Moreover, we develop an asymptotic expansion for the heat trace near $t=0$.
\end{abstract}

\section{INTRODUCTION}

On a compact manifold $M$ with boundary $\partial M$ which is the total space of a fiber bundle

$$
Z \hookrightarrow \partial M \stackrel{\pi}{\longrightarrow} Y,
$$

with $Z, Y$ closed manifolds, an incomplete cusp edge metric $g_{i c e}$ is, roughly speaking, a smooth Riemannian metric on the interior of $M$ which near the boundary takes the form

$$
g_{\text {ice }}=d x^{2}+x^{2 k} g_{Z}+\pi^{*} g_{Y}+\widetilde{g}, \quad k>1,
$$

where $g_{Y}$ is a metric on the base $Y, g_{Z}$ is positive definite restricted to the fibers, $x$ is the distance to the boundary (to first order), and $\widetilde{g}$ is a higher order term. Thus near the boundary $\left(M, g_{i c e}\right)$ is a bundle of geometric horns over a smooth Riemannian manifold $Y$. When $k=3$, such metrics model the singular behavior of the Weil-Petersson metric on the moduli space of Riemann surfaces, as we discuss below.

In this paper, we study the Hodge-Laplacian

$$
\Delta:=\Delta^{g_{i c e}}=d \delta+\delta d
$$

acting on differential forms. Our first result shows that under conditions which contain the main examples of interest, one need not impose 'ideal boundary conditions' at $\partial M$ in order to obtain a self-adjoint operator.

Theorem 1. Let $\left(M, g_{i c e}\right)$ be an incomplete cusp edge manifold that is 'Witt', meaning that either $\operatorname{dim} Z=f$ is odd or

$$
H^{f / 2}(Z)=\{0\}
$$

Assume furthermore that $g_{\text {ice }}$ satisfies 2.9 below and that the parameter $k$ in 1.2 satisfies

$$
k \geq 3 \text {. }
$$

The authors are happy to thank Rafe Mazzeo for helpful conversations, and to Richard Melrose for explaining to us his work with Xuwen Zhu on the Riemann moduli space. The authors would also like to thank the Max Planck Institute for Mathematics in Bonn for its support during the early stages of this project. 
Then the Hodge-Laplacian $\Delta^{g_{i c e}}$ acting on differential forms is essentially self-adjoint and has discrete spectrum.

Thus, by the spectral theorem [35, there exists an orthonormal basis of $L^{2}\left(\Omega^{p}(M)\right)$ of eigenforms $\Delta^{g_{i c e}} \alpha_{j, p}=\lambda_{j, p}^{2} \alpha_{j, p}$. We also prove that the distribution of eigenvalues satisfies "Weyl asymptotics," concretely, for fixed degree $p$

$$
\#\left\{j \mid \lambda_{j, p}^{2}<\lambda^{2}\right\}=c_{n} \operatorname{Vol}\left(M, g_{i c e}\right) \lambda^{n}+o\left(\lambda^{n}\right) \text { as } \lambda \rightarrow \infty .
$$

See $\$ 4.2$ for the proofs of Theorem 1 and of the asymptotic formula in (1.6).

Having established these fundamental properties of the Hodge-Laplacian on such spaces, we turn to the next natural topic: Hodge Theory. Here the object of study is "Hodge cohomology", or the space of $L^{2}$ harmonic forms,

$$
\mathcal{H}_{L^{2}}^{p}\left(M, g_{\text {ice }}\right)=\left\{\alpha \in L^{2}\left(\Omega^{p}(M), g_{\text {ice }}\right) \mid d \alpha=0=\delta \alpha\right\},
$$

and one phrasing of the Hodge theory problem is to find a parametrization for $\mathcal{H}_{L^{2}}^{*}\left(M, g_{i c e}\right)$ in terms of a topological invariant. As described in [17, in analogous settings the relevant topological space for Hodge theoretic statements is not the manifold $M$, but the stratified space $X$ obtained by collapsing the fibration at the boundary over the base,

$$
X:=M /\{p \sim q \mid p, q \in \partial M \text { and } \pi(p)=\pi(q)\} .
$$

In $\$ 4.3$ we will prove the following.

Theorem 2. For a cusp edge space $\left(M, g_{i c e}\right)$ whose link $Z$ satisfies the Witt condition (1.4), there is a natural isomorphism

$$
\mathcal{H}_{L^{2}}^{*}\left(M, g_{\text {ice }}\right) \simeq I H_{\overline{\mathfrak{m}}}(X)
$$

where $I H_{\overline{\mathrm{m}}}$ is the middle perversity intersection cohomology of $X$. Furthermore, differential forms $\gamma \in \mathcal{H}_{L^{2}}^{*}\left(M, g_{i c e}\right)$ admit asymptotic expansions at the boundary of $M$.

Moreover, if $Z \simeq \mathbb{S}^{f}$, the sphere of dimension $f$, then $X$ is homeomorphic to a differentiable manifold and the isomorphism (1.9) becomes

$$
\mathcal{H}_{L^{2}}^{*}\left(M, g_{i c e}\right) \simeq H_{d R}^{*}(X)
$$

where the latter is the de Rham cohomology of $X$.

We recall the relevant facts about intersection cohomology, originally defined by Goresky and MacPherson in [12, 13, in Section 4.3 below. The equivalence in (1.9) will follow using the arguments from Hunsicker and Rochon's recent work [19] on iterated fibered cusp edge metrics (which are complete, non-compact Riemannian manifolds). To elaborate on the asymptotic expansion for $L^{2}$-harmonic forms $\gamma$, we will show in Lemma 19 below that in fact

$$
\mathcal{H}_{L^{2}}^{*}\left(M, g_{i c e}\right)=\left\{\alpha \in L^{2}\left(\Omega^{p}(M), g_{i c e}\right) \mid \Delta^{g_{i c e}} \alpha=0\right\},
$$

(that the former is included into the latter is obvious), and we show that elements in the $L^{2}$ kernel of $\Delta^{g_{i c e}}$ have expansions at $\partial M$ analogous to Taylor expansions but with noninteger powers, a statement which can be be interpreted as a sort of elliptic regularity at the boundary of $M$.

One application of these results, and to putative further work we describe below, is to the analysis on the Riemann moduli spaces $\mathcal{M}_{\gamma, \ell}$ of Riemann surfaces of genus $\gamma \geq 0$ with $\ell \geq 0$ marked points. These spaces carry a natural $L^{2}$ metric, the Weil-Petersson metric $g_{W P}$, which near the interior of a divisor is an incomplete cusp edge metric with $k=3$. In general divisors may intersect with normal crossings, but in at least two cases only one divisor is present. 
Theorem 3. Let $\mathcal{M}_{1,1}$ (also known as the moduli space of elliptic curves) and $\mathcal{M}_{0,4}$ be the spaces of, respectively, once punctured Riemann surfaces of genus 1 and 4 times punctured Riemann surfaces of genus zero, modulo conformal diffeomorphism. Then the HodgeLaplacian $\Delta^{g_{W P}}$ on differential forms is essentially self-adjoint on $L^{2}$ with core domain $C_{c, o r b}^{\infty}$ (see Section 12) with discrete spectrum and Weyl asymptotics, and if $\overline{\mathcal{M}}_{1,1}$ and $\overline{\mathcal{M}}_{0,4}$ denote the Deligne-Mumford compactifications (see e.g. 16, 36). Then the de Rham cohomology spaces $H_{d R}\left(\overline{\mathcal{M}}_{1,1}\right)$ are naturally isomorphic to $\mathcal{H}_{L^{2}}^{*}\left(\overline{\mathcal{M}}_{1,1}, g_{W P}\right)$, and the same holds for $\overline{\mathcal{M}}_{0,4}$.

We discuss the proof at the end of Section 4.3 , though this is really a direct application of our results together with the recent work on the structure of the Weil-Petersson metric near a divisor in 26] and 28.

This article is partly motivated by Ji, Mazzeo, Müller, and Vasy's work 20] on the spectral theory of the (scalar) Laplace-Beltrami operator on the Riemann moduli spaces $\mathcal{M}_{g}$, for which it was shown by methods different from ours that it is essentially self-adjoint and its eigenvalues satisfy a Weyl asymptotic formula. Here they analyze incomplete cusp edge spaces with normal crossings, and find in particular that the value $k=3$ in 1.5 is critical; indeed for values $k<3$ one does not expect self-adjointness. It would be interesting (though more complex) to find a parametrization of the space of closed extensions of incomplete cusp edge Laplacians with $k<3$, which is expected to be infinite dimensional, e.g. by [3.

In contrast with [20, since our eventual goal is Hodge and index theory on moduli space, our main technical contribution is the construction and detailed description of the heat kernel $H=\exp \left(-t \Delta^{g_{\text {ice }}}\right)$. Indeed, our approach to establishing Theorem 1 (which justifies the use of the word 'the' in the previous sentence) and Theorem 2, is to develop in Theorem 12 below a precise understanding of the behavior of a fundamental solution to the heat equation, which we only conclude is the heat kernel after using it to prove Theorem 1; we establish asymptotic expressions for it at the singular set, uniformly down to time $t=0$, obtaining in particular in Corollary 18, an asymptotic formula for its trace (which has potential applications to index theory, since our method for analyzing $\Delta^{g_{\text {ice }}}$ may be used for other natural elliptic differential operators on these spaces as well) and fine mapping properties of $\Delta^{\text {gice }}$ which allow us to analyze its kernel, i.e. harmonic forms. This is all described in detail in Section 4 .

Essential self-adjointness of a differential operator $P$ is typically a statement about the decay of $L^{2}$ sections $u$ for which $P u \in L^{2}$. (Here the derivative is taken in the distributional sense.) The set of such sections is denoted

$$
\mathcal{D}_{\text {max }}:=\mathcal{D}_{\max }\left(\Delta^{g_{\text {ice }}}\right)=\left\{u \in L^{2} \mid P u \in L^{2}\right\} .
$$

This is the largest subset of $L^{2}$ which is a closed subspace in the graph norm $\|u\|_{\Gamma}=$ $\|u\|_{L^{2}}+\|P u\|_{L^{2}}$. On the other hand, the smallest such closed extension from the domain $C_{c}^{\infty}(M)$ is the closure, i.e. the minimal domain

$$
\mathcal{D}_{\text {min }}:=\mathcal{D}_{\text {min }}\left(\Delta^{g_{i c e}}\right)=\left\{u \in L^{2} \mid \exists u_{k} \in C_{c}^{\infty}(M) \text { with } \lim _{k \rightarrow \infty}\left\|u_{k}-u\right\|_{\Gamma}=0\right\} .
$$

The essential self-adjointness statement in Theorem 1 1 says that the smallest closed extension is equal to the largest, i.e. that

$$
\mathcal{D}_{\max }=\mathcal{D}_{\text {min }},
$$

and therefore there is exactly one closed extension. On the other hand, $\mathcal{D}_{\max }$ is dual to $\mathcal{D}_{\text {min }}$ with respect to $L^{2}$ and thus if 1.13 holds then $P$ with core domain $C_{c}^{\infty}(M)$ has exactly one closed extension, which we denote by $\mathcal{D}=\mathcal{D}_{\min }=\mathcal{D}_{\max }$ and $(P, \mathcal{D})$ is a self-adjoint, 
unbounded operator on $L^{2}$. Equation $(1.13)$ is a statement about decay in the sense that to prove it we will show that a differential form $\alpha \in \mathcal{D}_{\max }$ decays fast enough near $\partial M$ that it can be approximated in the graph norm by compactly supported smooth forms. This we do using the heat kernel.

Recall that the heat kernel $H$ is a section of the form bundle $\Pi$ : $\operatorname{End}(\Lambda) \longrightarrow M^{\circ} \times M^{\circ} \times$ $[0, \infty)$, where $M^{\circ}$ is the interior of $M$ and $\operatorname{End}(\Lambda)$ is the vector bundle whose fiber over $(p, q, t)$ is $\operatorname{End}\left(\Lambda_{q}^{*}(M) ; \Lambda_{p}^{*}(M)\right)$, smooth on the interior $M^{\circ} \times M^{\circ} \times[0, \infty)_{t}$, which solves

$$
\left(\partial_{t}+\Delta^{g_{i c e}}\right) H=0 \text { and } H_{t} \longrightarrow \mathrm{Id} \text {, strongly as } t \downarrow 0 \text {. }
$$

For a compactly supported smooth differential form $\alpha$, the differential form

$$
\beta(\omega, t):=\int_{M} H(w, \widetilde{w}, t) \alpha(\widetilde{w}) \mathrm{dVol}_{g_{i c e}}(\widetilde{w})
$$

solves the heat equation $\left(\partial_{t}+\Delta^{g_{i c e}}\right) \beta=0$ with initial data $\left.\beta\right|_{t=0}=\alpha$. One consequence of our precise description of $H$ in Theorem 12 below will be the following.

Theorem 4. On a Witt incomplete cusp edge space $\left(M, g_{i c e}\right)$ with metric satisfying the assumptions in (2.9) below together with (1.5), there exists a fundamental solution to the heat equation $H_{t}=H(w, \widetilde{w}, t)$ in the sense of 1.14 such that for $t>0$

$$
H_{t}: L^{2}\left(M ; \Omega^{*}(M)\right) \longrightarrow \mathcal{D}_{\min }
$$

and such that $H_{t}$ and $\partial_{t} H_{t}$ are bounded, self-adjoint operators on $L^{2}$.

Theorem 4 implies the essential self-adjointness statement; indeed the fundamental solution $H_{t}$ directly gives a sequence (indeed a path) of sections on $\mathcal{D}_{\text {min }}$ which approaches a given form in $\mathcal{D}_{\max }$. Namely,

$$
\alpha \in \mathcal{D}_{\max } \Longrightarrow H_{t} \alpha \rightarrow \alpha \text { in } \mathcal{D}_{\text {min }} \text { as } t \downarrow 0 .
$$

As we see now, the proof of this is straightforward functional analysis given the conclusions of Theorem 4 .

Proof of essential self-adjointness using Theorem 4. The proof has nothing to do with the fine structure of incomplete cusp edge spaces, it depends only on the soft properties of the fundamental solution $H$ in Theorem 4 4 . To emphasize this, let $(M, g)$ be any Riemannian manifold and $P$ a differential operator of order 2 acting on sections of a vector bundle $E$ with hermitian metric $G$, such that $P$ is symmetric on $L^{2}(M ; E)$. For $t>0$, let $H_{t}$ be a smooth section of $\operatorname{End}(E) \rightarrow M \times M$ which depends smoothly on $t$ and satisfies

$$
\left(\partial_{t}+P\right) H_{t}=0 \quad \text { and } \quad \lim _{t \rightarrow 0} H_{t}=\mathrm{Id},
$$

where the latter limit holds in the strong topology on $L^{2}$, and furthermore such that $H_{t}$ and $\partial_{t} H_{t}$ are self-adjoint on $L^{2}$.

Let $u \in \mathcal{D}_{\max }(P)$, i.e. $u \in L^{2}, P u \in L^{2}$. We will show that $u \in \mathcal{D}_{\min }(P)$ as well, and thus $\mathcal{D}_{\min }=\mathcal{D}_{\max }$. Indeed, we will show that

$$
H_{t} u \rightarrow u \text { in } \mathcal{D}_{\max }, \quad \text { i.e. that } H_{t} u \rightarrow u \text { and } P H_{t} u \rightarrow P u \text { in } L^{2} .
$$

This suffices to prove that $u \in \mathcal{D}_{\text {min }}$ since $H_{t} u \in \mathcal{D}_{\text {min }}$ by assumption and $\mathcal{D}_{\text {min }}$ is a closed subspace of $\mathcal{D}_{\max }$ in the graph norm. To prove (1.18), we note first that $H_{t} u \rightarrow u$ in $L^{2}$ trivially since $H_{t} \rightarrow \mathrm{Id}$ in the strong topology on $L^{2}$. Also note that since $u \in \mathcal{D}_{\max }$, $P u \in L^{2}$, so $H_{t} P u \rightarrow P u$ in $L^{2}$ also. Of course, this is not what we want; we want $P H_{t} u \rightarrow P u$, but in fact we claim that

$$
u \in \mathcal{D}_{\max } \Longrightarrow P H_{t} u=H_{t} P u,
$$


which will establish 1.13 .

It remains to prove (1.19). Note that for $u \in \mathcal{D}_{\max }$ and $v \in L^{2}$, then $\left\langle H_{t} P u, v\right\rangle_{L^{2}}=$ $\left\langle P u, H_{t} v\right\rangle_{L^{2}}$ by self-adjointness of $H_{t}$ on $L^{2}$, while $\left\langle P u, H_{t} v\right\rangle_{L^{2}}=\left\langle u, P H_{t} v\right\rangle_{L^{2}}$. Indeed, the adjoint domain of $\mathcal{D}_{\min }$ is $\mathcal{D}_{\max }$, so for any $f \in \mathcal{D}_{\min }, g \in \mathcal{D}_{\max },\langle P f, g\rangle_{L^{2}}=\langle f, P g\rangle_{L^{2}}$. But, then since $P H_{t}=-\partial_{t} H_{t}$ we see that $\left\langle H_{t} P u, v\right\rangle_{L^{2}}=-\left\langle u, \partial_{t} H_{t} v\right\rangle_{L^{2}}$. But $\partial_{t} H_{t}$ is self-adjoint on $L^{2}$ so $\left\langle u, \partial_{t} H_{t} v\right\rangle_{L^{2}}=\left\langle\partial_{t} H_{t} u, v\right\rangle_{L^{2}}=-\left\langle P H_{t} u, v\right\rangle_{L^{2}}$, and thus $\left\langle H_{t} P u, v\right\rangle_{L^{2}}=\left\langle P H_{t} u, v\right\rangle_{L^{2}}$ for all $u \in \mathcal{D}_{\max }, v \in L^{2}$, i.e. 1.19 holds.

The central vehicle for the construction of the heat kernel is the construction of a manifold with corners $M_{\text {heat }}^{2}$ via iterated radial blow up of the natural domain of the heat kernel, namely the space $M \times M \times[0, \infty)_{t}$; thus the interiors of these two spaces are diffeomorphic, and the blowup process furnishes a 'blowdown' map

$$
\beta: M_{\text {heat }}^{2} \longrightarrow M \times M \times[0, \infty)_{t},
$$

which encodes deeper information about the relationship between the various boundary hypersurfaces (codimension one boundary faces) of $M_{\text {heat }}^{2}$ and those of $M \times M \times[0, \infty)_{t}$. The upshot is that the heat kernel $H$, which lives a priori on the latter space, pulls back via $\beta$ to be "nice" (precisely to be polyhomogeneous, see Appendix A on $M_{\text {heat }}^{2}$. In fact, in Section 3 we will construct a parametrix $K$ for the heat equation directly on $M_{\text {heat }}^{2}$. To obtain the actual heat kernel $H$ we use a Neumann series argument to iterate away the error.

The latter process builds on what is now a substantial body of work on analysis (in particular the structure of heat kernels) on singular and non-compact Riemannian spaces, going back at least to the work of Cheeger on manifolds with conical singularities [7, 8, 9 . Our approach here is more closely related to Melrose's geometric microlocal analysis on asymptotically cylindrical manifolds [31] (a non-compact example) and Mooers' paper 32 ] on manifolds with conical singularities (an incomplete, singular example). The general procedure, which one sees in both the parabolic and elliptic settings, is to express the relevant differential operator as an element in the universal enveloping algebra of a Lie algebra of vector fields, and to 'resolve' this Lie algebra via radial blowup of the underlying space.

Two papers closely related to our work are, first, Mazzeo-Vertman 27, in which the authors study analytic torsion on incomplete edge spaces, which are the $k=1$ case of incomplete cusp edges. (They analyze the behavior of elliptic operators which have the same type of degeneracy in their principal symbols near the singular set as the Laplacian, so a weider class than considered here.) Their analysis involves a heat kernel construction using blowup analysis, which is slightly simpler in their context as the resolved double space has one less blow up (and thus the triple space is much simpler). One substantial difference between the incomplete edge and incomplete cusp edge cases is that in the incomplete edge case the space of self-adjoint extensions is substantially more subtle. In particular, a Witt space (this is a topological condition and has nothing to do with the value of $k$ ) that is incomplete edge may have infinitely many self-adjoint extensions if the family of induced operators on the fibers have small non-zero eigenvalues [3]; on incomplete cusp edge spaces the small non-zero fiber eigenvalues do not contribute. On the other hand, one expects that the zero mode in the fiber (the space of fiber harmonic forms) makes a simlar contribution in both the cusp and cone cases, in particular that an incomplete cusp edge space which is not Witt will have an infinite dimensional space of closed extensions on which 'Cheeger ideal boundary conditions' must be imposed to make the operator self-adjoint, as is the case in [3]. 
A second closely related work is Grieser-Hunsicker [15, which uses also inhomogeneous radial blowups, in this case to construct a Green's function for elliptic operators on a certain class of complete Riemannian manifolds (called ' $\phi$-manifolds') which require similar analysis. There are many other related works in a similar vein including, just to name a few, AlbinRochon [4, Brüning-Seeley 6], Gil-Krainer-Mendoza [11, Lesch [22, Schultze [34, and Grieser's notes on parametrix constructions for heat kernels [14. For analysis of moduli space, to give just a sample recent work, we refer the reader to the papers of Liu-Sun-Yao, for example [24, 23].

\section{CONTEnTS}

1. Introduction

2. Incomplete cusp edge differential geometry

2.1. Differential form bundles and the Hodge-Laplacian 7

2.2. Proof of Propositions 6 and 7

2.3. Fiber harmonic forms 11

3. The heat kernel 13

3.1. Heat double space 13

3.2. Model operators

3.3. Parametrix construction 23

4. Spectral and Hodge theoretic properties of the Hodge-Laplacian 27

4.1. Polyhomogeneous forms in $\mathcal{D}_{\max }$ and $\mathcal{D}_{\min }$

4.2. Spectral theory 30

4.3. Harmonic forms and Hodge theory 32

Appendix A. Manifolds with corners 34

A.1. Melrose's pushforward theorem 36

A.2. Heat trace asymptotics 37

Appendix B. Triple Space 38

References

\section{INCOMPLETE CUSP EDGE DIFFERENTIAL GEOMETRY}

As described above we work on a smooth manifold with boundary $M$ whose boundary is the total space of a fiber bundle with base $Y$ and typical fiber $Z$. (Again, we assume that $Y, Z$ are both compact and without boundary.) We fix once and for all a boundary defining function $x$, meaning a function $x \in C^{\infty}(M)$ with $\{x=0\}=\partial M$ and $d x$ non-vanishing on $\partial M$, and thereby fix (after possibly scaling $x$ by a constant) a tubular neighborhood of the boundary,

$$
\mathcal{U} \simeq \partial M \times[0,1)_{x} .
$$

Note that near each point $q$ on the boundary, there is a neighborhood $V$ in $\partial M$ of $y=\pi(q)(\pi$ is the projection onto the base) such that $\pi^{-1}(V) \simeq V \times Z$, where $\simeq$ denotes diffeomorphism. Below, we will say that we work 'locally over the base' when we fix our attention on a neighborhood of $q$ in $M$ of the form $\pi^{-1}(V) \times[0,1)_{x}$. If one chooses local coordinates $y$ on the base and $z$ on $\pi^{-1}(q) \simeq Z$, then

$$
(x, y, z) \text { form a coordinate chart on } M \text { in a neighborhood of } q \text {. }
$$


Let

$$
f:=\operatorname{dim} Z, \quad b:=\operatorname{dim} Y .
$$

In the space $X$ defined by collapsing the fibers $Z$ over the boundary (see $(1.8)$ ), the image of $\partial M$ via the projection $\pi: M \longrightarrow X$ defines a subset $\pi(\partial M)$, which can be identified with $Y$, and $\pi(\partial M)$ forms the unique singular stratum of the stratified space $X$. (See, for instance 2] or [10], among many others, for a discussion of stratified spaces.) In the case $Z \simeq \mathbb{S}^{f}$ a sphere, $X$ is homeomorphic to a manifold. In general, given $p \in \pi(\partial M)$, there is a basis of open sets at $p$ consisting of canonical neighborhoods homeomorphic to

$$
V \times C_{\delta}(Z)
$$

where $V$ is an open subset of $Y$ containing $p$ and

$$
C_{\delta}(Z)=[0, \delta) \times Z /\{0\} \times Z .
$$

2.1. Differential form bundles and the Hodge-Laplacian. We will consider differential forms and vector fields which are of approximately unit size with respect to Riemannian metrics of the type in (1.2). These are the incomplete cusp edge forms, which are sections of the incomplete cusp edge form bundle, ice $\Lambda^{*}(M)$, whose smooth sections are generated locally over the base by the forms

$$
d x, \quad d y_{i} \quad(i=1, \ldots, b=\operatorname{dim} Y), \quad x^{k} d z_{\alpha} \quad(\alpha=1, \ldots, f=\operatorname{dim} Z) .
$$

Locally over the base, we have the isomorphism

$$
{ }^{\text {ice }} \Lambda^{*}(M)=\Lambda^{*}(Y) \wedge x^{k \mathbf{N}} \Lambda^{*}(Z) \oplus d x \wedge \Lambda^{*}(Y) \wedge x^{k \mathbf{N}} \Lambda^{*}(Z)
$$

where $\mathbf{N}$ is a multiplication operator on $\Lambda^{*}(Z)$ which takes a form $\alpha$ of degree $q$ to $q \alpha$. Note that with respect to this decomposition, if $\mathbf{N}$ is the operator acting on $\Lambda^{\mathbf{N}}(Y)$ by multipliction by $\mathbf{N}$, the exterior derivative $d$ acts as

$$
d=\left(\begin{array}{cc}
d^{Y}+(-1)^{q_{Y}} x^{-k} d^{Z} & 0 \\
\partial_{x}+k x^{-1} \mathbf{N} & -d^{Y}-(-1)^{q_{Y}} x^{-k} d^{Z}
\end{array}\right) .
$$

Moreover, we will use the space of vector fields which are locally $C^{\infty}(M)$ linear combinations of the vector fields

$$
\partial_{x}, \quad \partial_{y_{i}}, \quad x^{-k} \partial_{z_{\alpha}}
$$

These vector fields are local sections of a bundle ${ }^{i c e} T(M)$ which is dual to ${ }^{i c e} T^{*}(M)=$ $\Lambda_{i c e}^{1}(M)$. We denote sections of ${ }^{i c e} T(M)$ by $\mathcal{V}_{i c e}$.

We consider metrics $g$ on $M$ which are sections of $\operatorname{Sym}^{0,2}\left({ }^{i c e} T^{*}(M)\right)$, and which locally over the boundary take the form

$$
\left.g=\left(\begin{array}{lll}
d x & d y^{i} & x^{k} d z^{\alpha}
\end{array}\right)\left(\begin{array}{ccc}
1 & 0 & 0 \\
0 & \left(h_{i j}\right) & 0 \\
0 & 0 & \left(\mathbf{k}_{\alpha \beta}\right)
\end{array}\right)+O\left(x^{k}, g_{0}\right)\right)\left(\begin{array}{c}
d x \\
d y^{i} \\
x^{k} d z^{\alpha}
\end{array}\right)
$$

with $h_{i j}$ and and $\mathbf{k}_{\alpha \beta}$ independent of $x$. Here $O\left(x^{k}, g_{0}\right)$ refers to a $O\left(x^{k}\right)$ norm bound with respect to an exact incomplete cusp edge metric $g_{0}$ as in 2.10 below, and furthermore we assume that the $O\left(x^{k}, g_{0}\right)$ term is polyhomogeneous conormal, a regularity assumption defined precisely in Section Abelow, which roughly speaking means that the coefficients have an asymptotic expansion at $x=0$ analogous to a Taylor expansion but with non-integer powers and with precise derivative bounds on the error terms. Metrics satisfying these assumptions are what we refer to henceforth as incomplete cusp edge metrics. (Note that the assumptions on $g$ are stronger than merely assuming that $g \in \operatorname{Sym}^{0,2}\left({ }^{i c e} T^{*}(M)\right)$, as the latter space contains e.g. $x\left(x^{k} d z \otimes_{\text {sym }} d x\right)$, which does not obey the error bound.) 
Remark 5. As is shown in 26] (see the introduction for further discussion) with previous results for example in [36, 37] the Weil-Petersson metric on moduli space takes the form (2.9) near the interior of a divisor and satisfies the polyhomogeneity assumption.

As an example of such a metric, one can take an exact incomplete cusp edge metric, constructed as follows. Let $h_{1} \in \operatorname{Sym}^{0,2}(y)$ be a metric on the base, and $h=\pi^{*} h_{1}$ its $x$ independent pullback via the projection $\pi: \partial M \longrightarrow Y$ extended to our tubular neighborhood $\mathcal{U} \simeq \partial M \times[0,1)_{x}$ in the obvious way. The tensor $\mathbf{k}$ we take to be the pullback to $\mathcal{U}$ of a tensor on $\partial M$ which by abuse of notation we write as $\mathbf{k} \in S y m^{0,2}(\partial M)$, having the property that $\mathbf{k}$ restricted to any fiber is positive definite. The metric $g_{0}=d x^{2}+h+x^{2 k} \mathbf{k}$ is an exact incomplete cusp edge metric. Note that in coordinates $(x, y, z)$ such a metric takes the form

$$
g_{0}=\left(\begin{array}{lll}
d x & d y^{i} & x^{k} d z^{\alpha}
\end{array}\right)\left(\begin{array}{ccc}
1 & 0 & 0 \\
0 & \left(h_{i j}\right) & x^{k}\left(\mathbf{k}_{i \alpha}\right) \\
0 & x^{k}\left(\mathbf{k}_{\alpha i}\right) & \mathbf{k}_{\alpha \beta}
\end{array}\right)\left(\begin{array}{c}
d x \\
d y^{i} \\
x^{k} d z^{\alpha}
\end{array}\right) .
$$

Thus an incomplete cusp edge metric diffes from an exact one by a polyhomogeneous error of order $O\left(x^{k}\right)$ in norm and can therefore be taken to correspond uniquely to an exact cusp edge metric.

Note that an exact incomplete cusp edge metric (and thus an incomplete cusp metric) gives rise, locally over the boundary to well defined notions of the operators $d_{Y}, d_{Z}$, the Hodge star operators $\star_{Y}$ and $\star_{Z}$, obtained by allowing $\star_{(Y, h)}$ the Hodge star associated with $(Y, h)$, the Riemannian structure on the base $Y$, to act on the $\Lambda^{*}(Y)$ factors in the decomposition (2.6) and $\star_{(Z, \mathbf{k})}$, the $y$-dependent Riemannian structure induced on the fibers, on the $x^{k \mathbf{N}} \Lambda^{*}(Z)$ factors. (So, for example, $\star_{Z} x^{k} d z_{\alpha}=x^{(f-1) k} \star_{Z} d z_{\alpha}$.) Thus, we may also define unambiguously (still locally over the boundary) the Hodge-de Rham operators $\partial_{Y}$ and $\widetilde{\partial}_{Z}$. Indeed, a homogeneous differential form $\alpha$, in the coordinates $(x, y, z)$ can be written $\alpha=\left(f(x, y, z) d y^{I}\right) \wedge x^{k p} d z^{A}(\wedge d x)= \pm\left(f(x, y, z) x^{k p} d z^{A}\right) \wedge d y^{I}(\wedge d x)$, where $I$ and $A$ are multi-indices and $|I|$, resp. $|A|$, is their order. One can act via $\partial_{Y}=d_{Y}+(-1)^{b(|I|-1)+1} \star_{Y}$ $d_{Y} \star_{Y}$ on $f(x, y, z) d y^{I}$, where $\star_{Y}$ is the Hodge star operator of $(Y, h)$, and thus define $\partial_{Y} \alpha$, or act via $\check{\partial}_{Z}=d_{Z}+(-1)^{f(|A|-1)+1} \star_{Z} d_{Z} \star_{Z}$ on $f(x, y, z) x^{k p} d z^{A}$ where $\star_{Z}$ is the Hodge star operator of $(Z, \mathbf{k})$, and thus define $\partial_{Z} \alpha$. Note that $\partial_{Z}$ is thus a family of operators on the fiber $Z$ parametrized by the base point $y$. The Hodge-de Rham operator can be decomposed locally over the boundary in terms of the base and fiber Hodge-de Rham operators according to the following proposition, which we prove in Section 2.2 below.

Proposition 6. Locally over the boundary (see below (2.1)), the Hodge-de Rham operator decomposes as

$$
\partial=\check{\partial}_{0}+\delta_{Z} P_{1}+P_{2} \delta_{Z}+x^{k-1} E,
$$

where $\mathrm{\partial}_{0}$ acts on sections of ${ }^{\text {ice }} \Lambda^{*}$ decomposed as in (2.6) by

$$
\partial_{0}=\left(\begin{array}{cc}
x^{-k} \partial^{Z}+\partial^{Y} & -\partial_{x}-k x^{-1}(f-\mathbf{N}) \\
\partial_{x}+k x^{-1} \mathbf{N} & -x^{-k} \partial^{Z}-\partial^{Y}
\end{array}\right),
$$

$\partial^{Z}$ depends on the base $Y$ parametrically, acts in only the fiber direction and only on the $\Lambda^{*}(Z)$ factor, and is equal to the Hodge-de Rham operator for the Riemannian manifold $\left.\boldsymbol{k}\right|_{y}$, and where the $P_{i}$ are polyhomogeneous, bounded endomorphisms on ${ }^{i c e} \Lambda^{*}$, and $E$ is as in (2.12)-2.14. 
To describe the term $E$, we say first it is a differential operator of order one which does not increase the order of blow up of polyhomogeneous distributions; in particular

$$
E\left(x^{k} \gamma\right)=O\left(x^{k}\right)
$$

for $\gamma$ a smooth, bounded section of ice $\Lambda^{*}$. In fact, it is a b-differential operators on ice-forms with polyhomogeneous coefficients

$$
E \in \operatorname{Diff}_{\mathrm{b}, \mathrm{phg}}^{1}\left(M ;{ }^{\text {ice }} \Lambda^{*}\right) .
$$

Letting $\mathcal{V}_{\mathrm{b}, \text { phg }}$ denote the polyhomogeneous vector fields tangent to the boundary $\partial M, 2.13$, means that $E$ lies in the algebra of differential operators generated $\mathcal{V}_{\mathrm{b}, \mathrm{phg}}$. Concretely, it satisfies

$$
E=a x \partial_{x}+b^{i} \partial_{y^{i}}+c^{\alpha} \partial_{z^{\alpha}}+d
$$

for polyhomogeneous, bounded endomorphisms $a, b^{i}, c^{\alpha}, d$, and where repeated indices are summed over. In general, an element $Q \in \operatorname{Diff}_{\mathrm{b}, \mathrm{phg}}^{m}\left(M ;{ }^{\text {ice }} \Lambda^{*}\right)$ also satisfies 2.12 , and is given locally by polyhomogeneous linear combinations of $x \partial_{x}, \partial_{y}, \partial_{z}$, i.e.

$$
Q=\sum_{i+|\alpha|+|\beta| \leq m} a_{i, \alpha, \beta}\left(x \partial_{x}\right)^{i} \partial_{y}^{\alpha} \partial_{z}^{\beta},
$$

where $a_{i, \alpha, \beta}$ is a polyhomogeneous bounded endomorphism of ${ }^{\text {ice }} \Lambda^{*}$.

The Hodge-Laplacian $\Delta=\partial^{2}=d \delta+\delta d$ can now be decomposed along the same lines.

Proposition 7. Locally over the base, $\Delta$ can be decomposed as follows

$$
\Delta=\Delta_{0}+x^{-k} \widetilde{P}+x^{-1} \widetilde{E},
$$

where $\Delta_{0}$ acts on forms decomposed as in (2.6) by

$$
\begin{aligned}
\Delta_{0}=\mathrm{Id}_{2 \times 2}\left(-\partial_{x}^{2}-\frac{k f}{x} \partial_{x}+\frac{1}{x^{2 k}} \Delta^{Z, y}+\Delta^{Y}\right. & \\
& +\left(\begin{array}{cc}
k \mathbf{N}(1-k(f-\mathbf{N})) x^{-2} & -2 k x^{-k-1} d^{Z} \\
-2 k x^{-k-1} \delta^{Z} & k(f-\mathbf{N})(1-k \mathbf{N}) x^{-2}
\end{array}\right),
\end{aligned}
$$

where

$$
\widetilde{P}=\delta_{Z} Q_{1}+d_{Z} Q_{2}+Q_{3} \delta_{Z}+Q_{4} d_{Z}
$$

and where the $Q_{i} \in \operatorname{Diff}_{\mathrm{b}, \mathrm{phg}}^{1}(M)$ and $\widetilde{E} \in \operatorname{Diff}_{\mathrm{b}, \mathrm{phg}}^{2}(M)$, as described below Proposition 6 .

2.2. Proof of Propositions 6 and 7. To prove Proposition 6, we will compare the Hodgede Rham operator for $g$ to that of the locally defined warped product metric

$$
g_{p}=\left(\begin{array}{lll}
d x & d y^{i} & x^{k} d z^{\alpha}
\end{array}\right)\left(\begin{array}{ccc}
1 & 0 & 0 \\
0 & \left(h_{i j}\right) & 0 \\
0 & 0 & \left(\mathbf{k}_{\alpha \beta}\right)
\end{array}\right)\left(\begin{array}{c}
d x \\
d y^{i} \\
x^{k} d z^{\alpha}
\end{array}\right) .
$$

We compute the Hodge-de Rham operator for $g_{p}$ now. First of all, the Hodge star operator of $g_{p}, \star_{p}$, acts on forms decomposed as in (2.6) as

$$
\star_{p}=\left(\begin{array}{cc}
0 & (-1)^{\mathbf{N}\left(b-q_{Y}\right)} \star_{Y} \star_{Z} \\
(-1)^{q_{Y}+\mathbf{N}\left(b-q_{Y}+1\right)} \star_{Y} \star_{Z} & 0
\end{array}\right),
$$

where $q_{Y}$ denotes multiplication by the degree in $Y$ and $\mathbf{N}$ denotes multiplication by the degree in $Z$, and $b=\operatorname{dim} Y$. Using (2.7), we see that the Hodge-de Rham operator for $g_{p}$ acting on $k$-forms, $\check{\partial}_{p}=d+(-1)^{k(n-k)} \star_{p} d \star_{p}$ satisfies (2.11). We now use this to prove the proposition. 
Proof of Proposition 6. A brief calculation shows that (2.11) holds with $P_{1}=P_{2}=E=0$ for the warped product metric $g_{p}$, defined locally on $M$ in (2.17). Next, we claim that the general case follows from that of a warped product metric $g_{p}$. To see this, it suffices to show that if $\star_{p}$ continues to denote the Hodge star operator for $g_{p}$, then the pointwise norm

$$
\left\|\star_{p}-\star\right\|_{g} \leq C x^{k} .
$$

Indeed, if this holds, then writing

$$
\pm \delta=\star\left(d x \wedge \partial_{x} \oplus d_{Y} \oplus d_{Z}\right) \star,
$$

we consider $\delta-\delta_{g_{p}}$, the latter being the dual of $d$ with respect to $d_{g_{p}}$, which is given by \pm 1 times

$$
\left(\star d x \wedge \partial_{x} \star-\star_{p} d x \wedge \partial_{x} \star_{p}\right) \oplus\left(\star d_{Y} \star-\star_{p} d_{Y} \star_{p}\right) \oplus\left(\star d_{Z} \star-\star_{p} d_{Z} \star_{p}\right) .
$$

The operator $d_{Z}$, as an operator on sections of ${ }^{\text {ice }} \Lambda^{*}$ satisfies $d_{Z}=\sum a_{i} \partial_{z_{i}}$ where $a_{i}$ are endomorphisms satisfying $a_{i}=O\left(x^{-k}\right)$. (Proof: $d_{Z} \alpha=\sum_{\alpha}\left(x^{k} d z_{\alpha}\right) \wedge x^{-k} \partial_{z_{\alpha}} \alpha$ and $x^{k} d z_{i}$ is a bounded endomorphism since $x^{k} d z_{i}$ has unit length.) By the same rationale, $d x \wedge \partial_{x}$ and $d_{Y}$ are differential operators with bounded coefficients. By $(2.19)$ it follows that in the difference

$$
\begin{aligned}
\delta-\delta_{p} & = \pm\left(* d *-*_{p} d *_{p}\right) \\
& = \pm \delta d\left(*-*_{p}\right) \pm\left(*-*_{p}\right) d\left(*-*_{p}\right) \pm\left(*-*_{p}\right) d * \\
& = \pm \delta *\left(*-*_{p}\right) \pm\left(*-*_{p}\right) d\left(*-*_{p}\right) \pm\left(*-*_{p}\right) * \delta
\end{aligned}
$$

the terms coming from $d_{Y}$ and $\partial_{x}$ derivatives have coefficients bounded to order $x^{k}$, while those coming from $d_{Z}$ have coefficients with bounded derivatives. Hence (2.11) follows.

It remains to prove 2.19 . First note that, under the assumptions above, if we let $g^{\otimes k,-\otimes l}=g \otimes \cdots \otimes g \otimes g^{-1} \otimes \cdots \otimes g^{-1}$ denote the metric induced by $g$ on $T M^{\otimes k} \otimes T^{*} M^{\otimes l}$, and similarly for $g_{p}$, then

$$
\left\|g^{\otimes k,-\otimes l}-g_{p}^{\otimes k,-\otimes l}\right\|_{g}<C x^{k}
$$

where the norm is the one induced by $g$ on the tensor powers. Indeed this is obvious for $l=0$. Moreover, $g=g_{p}+O\left(x^{k}\right)$ implies $g^{-1}=g_{p}^{-1}+O\left(x^{k}\right)$, and 2.22 follows. Now let $e_{i}$ be an oriented orthonormal basis of 1 -forms for $g$, and for $I=\left(i_{1}, \ldots, i_{k}\right)$ denoting an ordered subset of $\{1, \ldots, n\}$, let $e_{I}=e_{i_{1}} \wedge \cdots \wedge e_{i_{k}}$ be the corresponding $k$-form. Then $\star e_{I}=e_{I^{c}}$ where $I^{c}$ denotes the complement of $I$ in $\{1, \ldots, n\}$ such that $e_{I} \wedge e_{I^{c}}$ is positive. Thus, we want to show that $\left\|\star e_{I}-\star_{p} e_{I}\right\|_{g}<c x^{k}$. For two ordered multi-indices $I, J$, letting $\delta_{I, J}=1$ if $I=J$ and zero otherwise, consider

$$
\begin{aligned}
\left\langle\star_{p} e_{I}, e_{J}\right\rangle_{g_{p}} & =\left\langle\left\langle\star_{p} e_{I}, e_{J}\right\rangle_{g_{p}} \mathrm{dVol}_{g_{p}}, \mathrm{dVol}_{g_{p}}\right\rangle_{g_{p}} \\
& =\left\langle e_{I} \wedge e_{J}, \mathrm{dVol}_{g_{p}}\right\rangle_{g_{p}} \\
& = \pm \delta_{I^{c}, J}\left\langle\mathrm{dVol}_{g}, \mathrm{dVol}_{g_{p}}\right\rangle_{g_{p}} \\
& = \pm \delta_{I^{c}, J}\left(1+O\left(x^{k}\right)\right)
\end{aligned}
$$

so since $\left\langle\star_{p} e_{I}, e_{J}\right\rangle_{g}=\left\langle\star_{p} e_{I}, e_{J}\right\rangle_{g_{p}}+O\left(x^{k}\right)= \pm \delta_{I^{c}, J}\left(1+O\left(x^{k}\right)\right)=\left\langle\star e_{I}, e_{J}\right\rangle_{g}+O\left(x^{k}\right)$, the bound in 2.19 holds.

To see that Proposition 7 holds, simply use $\Delta=ð^{*} \precsim$ together with Proposition 6 . 
2.3. Fiber harmonic forms. We now discuss forms which are approximately harmonic with respect to the family of metrics $\mathbf{k}$ induced on the fibers $Z$ by an ice-metric as in (2.9). We begin by working directly on the fiber bundle $\pi: \partial M \longrightarrow Y$ with a submersion metric, i.e. a metric $g^{\partial}=\pi^{*} h_{1}+\mathbf{k}$ (so $h_{1}$ is a Riemannian metric on $Y$ and $\mathbf{k}$ is assumed only to be non-degenerate when restricted to the fibers). Then $\alpha \in \Omega^{q}(\partial M)$ is fiber harmonic if $\left.\alpha\right|_{\pi^{-1}(p)}$ is harmonic with respect to $\left.\mathbf{k}\right|_{\pi^{-1}(p)}$ for every $p \in Y$, i.e. if it lies in the kernel of $\Delta^{Z_{y}, \mathbf{k}_{y}}$. One can obtain fiber harmonic forms as follow. Let $\widetilde{\alpha} \in \Gamma\left(Y ; \Omega_{f i b}^{q}\right)$, where $\Omega_{f i b}^{q}$ is the bundle whose fiber over a point $p \in Y$ is the space of $q$-forms on $\pi^{-1}(p)$. Since a metric $g^{\partial}$ is present, there is a natural way to extend such a $\widetilde{\alpha}$ to a differential form on $\partial M$; consider the space $H^{q}:=L^{2}\left(\pi^{*} \Omega^{q}(Y)\right) \subset L^{2}\left(\partial M ; \Lambda^{q}\right)$ which is the $L^{2}$ closure of the space of differential forms pulled back from $Y$, and note that there is a unique differential form $\widetilde{\alpha}_{V}$ on $\partial M$ which is at every point orthogonal to $H^{q}$ and which pulls back to $\widetilde{\alpha}$ on each fiber. (This is analogous to being a horizontal vector field; vertical vector fields are defined in differential terms and the horizontal vector fields are defined with the metric. Indeed, being a vertical form here means being dual via the metric to a vertical vector field). Moreover, if $\widetilde{\alpha}$ is harmonic on each fiber, we claim that $\alpha:=\widetilde{\alpha}_{V}$ is fiber harmonic. Namely, choosing a local trivialization of $\partial M$ near $p \in Y$ and coordinates $(y, z)$, for a family of $q$-forms $\widetilde{\alpha}$ on the fibers, the vertical extension satisfies

$$
\widetilde{\alpha}_{V}=\sum_{|A|=q} a^{A} d z_{A}+\sum_{|I|+|B|=q,|I|>1} b^{I, B} d y_{I} \wedge d z_{B},
$$

with $\widetilde{\alpha}=\sum_{|A|=q} a^{A} d z_{A}$. Choosing another local trivialization $(\widetilde{y}, \widetilde{z})$ we have $z=z(\widetilde{y}, \widetilde{z}), y=$ $\widetilde{y}, d z_{i}=\left(\partial z^{i} / \partial \widetilde{z}^{j}\right) d \widetilde{z}^{j}+f^{i} d y_{i}$. Thus the pullback of $\widetilde{\alpha}_{V}$ is the change of variables for differential forms on the fiber, on which $\Delta^{Z, y}$ acts invariantly, proving the claim.

Let $\operatorname{ker}\left(\Delta^{Z}\right) \longrightarrow Y$ denote the bundle whose fiber above each $y$ is the kernel of $\Delta^{Z_{y}, \mathbf{k}_{y}}$. This bundle has a grading by the form degree,

$$
\mathcal{H}_{\partial}^{q}:=\operatorname{ker}\left(\Delta^{Z}: \Omega^{q}(Z) \longrightarrow \Omega^{q}(Z)\right),
$$

and by the above paragraph the vertical extensions of these forms also forms a vector bundle $\mathcal{H}_{\partial, V}^{q} \subset \Lambda^{q}(\partial M)$, the sections of which are fiber harmonic. We now define the approximately fiber harmonic differential forms $\mathcal{H}$ to be the direct sum of the spaces

$$
\mathcal{H}=\oplus_{q=0}^{f} \mathcal{H}^{q}, \text { where } \mathcal{H}^{q}:=\pi^{*} \Lambda(Y) \wedge x^{k q} \mathcal{H}_{\partial, V}^{q},
$$

or, in words, the sections of $\mathcal{H}^{q}$ form the space of differential forms on $\mathcal{U} \simeq \partial M \times[0,1)_{x}$ pulled back from $Y$ wedged with the vertical extentions of harmonic $q$-forms on the fibers, thought of as living on $\mathcal{U}$ and weighted by $x^{k q}$ (so as to make them unit size.)

The main point to note about sections of $\mathcal{H}$ in 2.25) is that a form $\gamma \in \mathcal{H}$ satisfies that, locally over the base, we have

$$
\Delta^{Z, y} \gamma=O\left(x^{k}\right)
$$

where $\Delta^{Z, y}$ is the operator defined (again only locally over the base) in Proposition 7 , and as usual the $O\left(x^{k}\right)$ bound is a pointwise norm bound. Indeed, this follows since by (2.24), under a change of local trivialization of the boundary fibration, we have

$$
x^{k} d z_{i}=x^{k}\left(\partial z^{i} / \partial \widetilde{z}^{j}\right) d \widetilde{z}^{j}+O\left(x^{k}\right),
$$

so $x^{k q}$ times $\gamma$ is $O\left(x^{k}\right)$ times the $q$-form part of the pullback to the fiber, together with the fact that the $q$-form part of the pullback is harmonic.

Below we will often work with forms which are merely polyhomogeneous (as opposed to smooth). These are sections $\gamma$ of $\mathcal{A}_{\mathrm{phg}}\left({ }^{\text {ice }} \Lambda^{*}\right.$ ), defined rigorously in (A.3) below. As described 
above, these have an expansion at $x=0$ analogous to a Taylor expansion, but with noninterger and possibly also $\log ^{p}(x)$ factors. In particular we will be forced by possibly only polyhomogeneous regularity of the metric to work in the larger space $x^{s_{0}} \mathcal{A}_{\text {phg }}\left(\mathcal{H}^{q}\right)$ defined to be the subset of $\mathcal{A}_{\mathrm{phg}}\left({ }^{\mathrm{ice}} \Lambda^{*}\right)$ such that

$$
x^{s_{0}} \mathcal{A}_{\mathrm{phg}}\left(\mathcal{H}^{q}\right) \subset x^{s_{0}} C^{\infty}\left(M ; \mathcal{H}^{q}\right) \oplus O\left(x^{s_{0}+k}\right) .
$$

This space contains, in particular, sections of $x^{s_{0}} \mathcal{H}^{q}$, and also ice-forms $\gamma$ which are polyhomogeneous and can be written as $\widetilde{\gamma}=x^{s_{0}} \gamma_{0}+O\left(x^{s_{0}+k}\right)$, where $\gamma_{0} \in \mathcal{H}^{q}$, but moreover contains $x^{s_{0}} \gamma_{1}+x^{s_{0}+1 / 2} \gamma_{2}+O\left(x^{s_{0}+k}\right)$ for $\gamma_{i} \in \mathcal{H}^{q}$ and indeed any polyhomogeneous iceform whose expansion terms not bounded in norm by $x^{s_{0}+k}$ have fiber harmonic coefficients. Thus, $\gamma \in x^{s_{0}} \mathcal{A}_{\text {phg }}\left(\mathcal{H}^{q}\right)$ implies that locally over the base,

$$
\Delta^{Z, y} \widetilde{\gamma}=O\left(x^{s_{0}+k}\right) .
$$

It is in fact possible to define an operator $\Delta_{f i b}$ on certain forms which is an invariant version of the locally defined $\Delta^{Z, y}$, for example on smooth sections of ice $\Lambda^{*}(M)$, if one notices that a section $\alpha \in C^{\infty}\left(M ;{ }^{\text {ice }} \Lambda^{*}(M)\right)$ defines, by restriction to the boundary, a smooth section of $\Lambda^{*} Y \otimes \Omega_{f i b}^{*} \longrightarrow Y$. Indeed, this follows since 2.27) and local computation produces a form on $Y$ with values in $\Omega^{*}(Z)$. Letting $\widetilde{\alpha}$ denote this section, one can then define $\alpha$ to be $\beta_{1}=x^{k} \mathbf{N}\left(\Delta^{Z, y} \widetilde{\alpha}\right)_{V}$, the vertical extension of the fiber-wise Laplacian applied to the $\Omega^{*}(Z)$ factor of $\widetilde{\alpha}$, and weighted by $x^{k \mathbf{N}}$ to make it a smooth section of ice $\Lambda^{*}(M)$. Then $\beta_{1}-\Delta^{Z, y} \alpha=O(x)$ locally over the base. One can in fact iterate this to find a section $\Delta_{f i b} \alpha:=\beta$ such that $\beta-\Delta^{Z, y} \alpha=O\left(x^{k}\right)$, since if $x \alpha^{\prime}$ is a smooth section then letting $\widetilde{\alpha}^{\prime}$ denote its value (as an $\Omega^{*}(Z)$-valued form on $Y$ ) the ice-form $\beta_{2}=x \cdot x^{k \mathbf{N}}\left(\Delta^{Z, y} \widetilde{\alpha}^{\prime}\right)_{V}$, then $\left(\beta_{1}+\beta_{2}\right)-\Delta^{Z, y} \alpha=O\left(x^{2}\right)$, and this can be iterated up to the error $O\left(x^{k}\right)$ (and since all steps introduce $O\left(x^{k}\right)$ errors, no better.) We will let $\Delta_{f i b} \alpha=\beta$.

We will also need to ask when a, say, smooth ice-form can be written as $\Delta_{f i b}$ of another form, or equivalently, when, given a ice-form $\alpha$, there exists a ice $\Lambda^{*}$-form $\gamma$ such that locally over the base $\alpha-\Delta^{Z, y} \gamma=O\left(x^{k}\right)$. A related question is whether there is an invariant definition of projection onto the space of fiber harmonic forms. Indeed, given $\alpha$ a smooth section of ice $\Lambda^{*}(M)$, again considering the $\Omega_{f i b}^{*}$ valued form on $Y$, $\widetilde{\alpha}$, over the boundary, if $\Pi^{y}$ denotes the projection onto the kernel of $\Delta^{Z, y}$, we can consider $x^{k} \mathbf{N}\left(\Pi^{y} \widetilde{\alpha}\right)$, and again can iterate this $k$-steps down the Taylor expansion of $\alpha$ to obtain a projection $\Pi^{\mathcal{H}}$ onto the fiber harmonic forms. In particular, if $\alpha \in x^{s_{0}} \mathcal{H}_{p h g}$, the definition of $\Pi^{\mathcal{H}}$ can be extended to

$$
\Pi_{\mathcal{H}}: x^{s_{0}} L^{2}\left({ }^{\text {ice }} \Lambda^{*}\right) \longrightarrow x^{s_{0}} \mathcal{H}, \quad \Pi_{\mathcal{H}}=\Pi_{\mathcal{H}}^{\text {loc }}+O\left(x^{k}\right),
$$

where $\Pi_{\mathcal{H}}^{l o c}$ is the projection onto $\operatorname{ker}\left(\Delta^{Z, y}\right)$ defined locally over the base, so that, again locally over the base we have

$$
\Pi^{\mathcal{H}} d^{Z}, \Pi^{\mathcal{H}} \delta^{Z}, d^{Z} \Pi^{\mathcal{H}}, \delta^{Z} \Pi^{\mathcal{H}} \in x^{k} \operatorname{Diff}_{\mathrm{b}}^{1}(M),
$$

and thus since $\Delta^{Z, y}=d^{Z} \delta^{Z}+\delta^{Z} d^{Z}$,

$$
\Pi^{\mathcal{H}} \Delta^{Z, y} \Pi^{\mathcal{H}} \in x^{2 k} \operatorname{Diff}_{\mathrm{b}}^{1}(M),
$$

where $\operatorname{Diff}_{\mathrm{b}}^{1}$ is defined in (2.13). Moreover, it is straightforward to show that if $\alpha$ is a smooth section of $x^{s_{0}}\left({ }^{\text {ice }} \Lambda^{*}(M)\right)$ then

$$
\Pi^{\mathcal{H}} \alpha=O\left(x^{s_{0}+k}\right) \Longrightarrow \exists \gamma \text { such that } \Delta^{Z, y} \gamma-\alpha=O\left(x^{s_{0}+k}\right),
$$

this holding locally over the base, with the form $\gamma$ defined globally. 


\section{THE HEAT KERNEL}

In this section we construct a manifold with corners $M_{\text {heat }}^{2}$ as in 1.20 together with a fundamental solution to the heat equation which is a polyhomogeneous conormal distribution on $M_{\text {heat }}^{2}$ with prescribed leading order terms in its asymptotic expansions at the various faces (see Theorem 12). To do so, after the construction of $M_{\text {heat }}^{2}$, we perform a parametrix construction and then use this parametrix to obtain the fundamental solution itself via a Neumann series.

3.1. Heat double space. The space $M_{\text {heat }}^{2}$ is obtained by performing three consecutive inhomogeneous radial blowups of $M \times M \times[0, \infty)_{t}$. Such blowups create, from a given manifold with corners $X$ and some other data including a submanifold $N$, another manifold with corners $[X ; N]_{\text {inhom }}$, which is diffeomorphic to the complement of $N_{+}(N)$. Here $N_{+}(N)$ denotes the inward-pointing normal bundle of $N$ which we think of as an open neighborhood of $N$ in $X$; thus $[X ; N]_{\text {inhom }}$ is a manifold with corners with one more boundary hypersurface (bhs) than $X$. It comes together with a blowdown map $\beta:[X ; N]_{\text {inhom }} \longrightarrow X$ which is a diffeomorphism of the interiors and satisfies that $C^{\infty}\left([X ; N]_{\text {inhom }}\right)$ contains $\beta^{*} C^{\infty}(X)$ properly, i.e. there are functions which are not smooth on $X$ but nonetheless pull back to smooth functions on the blown up space.

In more detail, the submanifold $N$ is a 'p-submanifold,' meaning that there are local coordinates $\left(x, x^{\prime}, y, y^{\prime}\right) \in\left(\mathbb{R}^{+}\right)_{\left(x, x^{\prime}\right)}^{k} \times \mathbb{R}_{\left(y, y^{\prime}\right)}^{n-k}$ with $x \in\left(\mathbb{R}^{+}\right)^{p+1}, y \in \mathbb{R}^{q}$ in which $N$ is defined (locally) by $x=0, y=0$. The space $[X, N]_{\text {inhom }}$ with homogeneities $x_{0} \sim x_{1}^{\alpha_{1}} \sim \cdots \sim x_{p}^{\alpha_{p}} \sim$ $y_{1}^{\beta_{1}} \sim \cdots \sim y_{q}^{\beta_{q}}$ with $\alpha_{j}, \beta_{k} \in \mathbb{N}, 1 \leq \alpha_{1} \leq \cdots \leq \alpha_{p}, \beta_{1} \leq \cdots \leq \beta_{q}$ is a manifold with corners whose set of boundary hypersurfaces contains that of $X$ naturally, and $[X, N]_{\text {inhom }}$ has one new boundary hypersurface, which we call nf for 'new face'. Assuming for the moment that $\alpha_{p} \geq \beta_{q}$ and also that $\alpha_{p}\left|\alpha_{j}, \alpha_{p}\right| \beta_{k}$ for all $j, k$, the space $\mathcal{M}\left([X, N]_{\text {inhom }}\right)$ is by definition the set $X \backslash N \cup \Gamma$ where $\Gamma$ is the set of paths $\gamma(s)$ in $X$ with $\gamma(0) \in N$ satisfying that $x_{p}=a_{p}(s) s$ for smooth non-vanishing $s$, and $x_{j}=a_{j}(s) s^{\alpha_{p} / \alpha_{j}}, y_{k}=b_{k}(s) s^{\alpha_{p} / \beta_{k}}$ for smooth $a_{j}, b_{k}$, all other coordinates being smooth functions of $s$, modulo the equivalence relation $\gamma_{1} \sim \gamma_{2}$ if the coordinate functions agree to order higher then the stated vanishing order (e.g. $x_{j}\left(\gamma_{1}(s)\right)-x_{j}\left(\gamma_{2}(s)\right)=O\left(s^{\left(\alpha_{p} / \alpha_{j}\right)+1}\right)$. The space $X \backslash N \cup \Gamma$ is naturally isomorphic to $X \backslash N \cup N^{+}(N)$ and can be given a smooth structure so that the following polar coordinates are smooth and valid on a collar neighborhood of the introduced face nf,

$$
\begin{aligned}
& \rho_{\mathrm{nf}}=\left(x_{0}+x_{1}^{\alpha_{1}}+\cdots+x_{p}^{\alpha_{p}}+\left|y_{1}\right|^{\beta_{1}}+\cdots+\left|y_{q}\right|^{\beta_{q}}\right)^{1 / \alpha_{p}}, \\
& \phi_{\mathrm{nf}}=\left(\frac{x_{0}}{\rho_{\mathrm{nf}}^{\alpha_{p}}}, \frac{x_{1}}{\rho_{\mathrm{nf}}^{\alpha_{p} / \alpha_{1}}}, \ldots, \frac{y_{q}}{\rho_{\mathrm{nf}}^{\alpha_{p} / \beta_{q}}}\right) .
\end{aligned}
$$

A full set of (polar) coordinates is then $\left(\rho_{\mathrm{nf}}, \phi_{\mathrm{nf}}, x^{\prime}, y^{\prime}\right)$

For a detailed definition of such spaces we refer to Melrose's work [29, Chapter 5] which contains a more general construction which does not assume that one has in particular a fixed extension for the manifold $N$ away from the boundary, (whereas here we fix once and for all a boundary defining function $x$ as in 2.1 , which will give all the desired extensions below). See also [15, 21].

(1) We first blow up the fiber diagonal in the corner. This is the subset of $\partial M \times \partial M \times$ $\{0\} \subset M \times M \times[0, \infty)_{t}$ consisting of points $(p, q, 0)$ with $\pi(p)=\pi(q)$ where $\pi$ is the projection of the fibration $\partial M$ onto its base. If local coordinates $(x, y, z)$ are chosen as above, this set can be written

$$
\mathcal{B}_{0}:=\left\{x=\widetilde{x}=t=\operatorname{dist}_{h}(y, \widetilde{y})=0\right\},
$$


where $\operatorname{dist}_{h}\left(\bullet, \bullet^{\prime}\right)$ is the distance function on the base $(Y, h)$. In fact, $\mathcal{B}_{0}$ is naturally isomorphic to $\operatorname{diag}_{\text {fib }}(\partial M) \times\{0\}$ where $\operatorname{diag}_{f i b}(\partial M)$ is the "fiber diagonal," i.e. the fiber product of $\partial M \times_{\text {fib }} \partial M$. We let

$$
M_{\text {heat }, 1}^{2}:=\left[M \times M \times[0, \infty)_{t} ; \mathcal{B}_{0}\right]_{\text {inhom }},
$$

with $t \sim x^{2} \sim \widetilde{x}^{2} \sim|y-\widetilde{y}|^{2}$. To be precise, $M_{\text {heat }, 1}^{2}$ is the parabolic blowup in time of the set $\mathcal{B}_{0}$ as defined in [31, Chapter 7]. In particular there is a blowdown map $\beta_{1}: M_{\text {heat }, 1}^{2} \longrightarrow M^{2} \times[0, \infty)_{t}$, and polar coordinates on $M_{\text {heat }, 1}^{2}$ near $\beta_{1}^{-1}\left(\mathcal{B}_{0}\right)$ (once coordinates $y, z$ are chosen on $\partial M$ ) are given by

$$
\begin{aligned}
\rho & =\left(t+x^{2}+\widetilde{x}^{2}+|y-\widetilde{y}|^{2}\right)^{1 / 2}, \\
\phi & =\left(\frac{t}{\rho^{2}}, \frac{x}{\rho}, \frac{\widetilde{x}}{\rho}, \frac{y-\widetilde{y}}{\rho}\right) \\
& =\left(\phi_{t}, \phi_{x}, \phi_{\widetilde{x}}, \phi_{y}\right), \text { along with } \widetilde{y}, z, \widetilde{z} .
\end{aligned}
$$

The set $\{\rho=0\}$ is a boundary hypersurface on $M_{\text {heat, } 1}^{2}$ introduced by the blow up; we call it $\mathrm{ff}_{1}$; we will see that only the projection of the heat kernel onto the zero mode in $Z$ is relevant at the face $\mathrm{ff}_{1}$. Letting $s=x / \widetilde{x}$, the interior of $\mathrm{ff}_{1}$ is the total space of a fiber bundle over $Y \times(0, \infty)_{s}$, which is the fiber product $\partial M \times_{\text {fib }} \partial M \times_{\text {fib }} T Y \times \mathbb{R}_{t^{\prime}}$ where $t^{\prime}$ is a rescaled time variable (see (3.5) below). Indeed, the map from $\mathrm{ff}_{1}$ to the base $Y$ is simply $\left.\beta_{1}\right|_{\mathrm{ff}_{1}}$

(2) The preceding blow up does not resolve the term $\frac{t}{x^{2 k}} \Delta^{Z, y}$ arising from 2.16). To accomplish this, we blow up the subset of $\mathrm{ff}_{1}$ defined in polar coordinates by

$$
\mathcal{B}_{1}:=\left\{\rho=0, \phi_{t}=\phi_{y}=0, \phi_{x}=\phi_{\widetilde{x}}\right\},
$$

i.e. by $\rho=0, \phi=(0,1 / \sqrt{2}, 1 / \sqrt{2}, 0)$, inhomogeneously so that near the new face, $\mathrm{ff}$, the function $t / x^{2 k}$ is smooth, and furthermore so that $t \partial_{x}^{2}$ is non-degenerate, the latter condition being satisfied if $(x-\widetilde{x}) / \sqrt{t}$ is smooth up to the interior of ff. Near $\mathcal{B}_{1}$ we can use projective coordinates

$$
\widetilde{x}, \quad s=x / \widetilde{x}, \quad \eta=\frac{y-\widetilde{y}}{\widetilde{x}}, \quad t^{\prime}=t / \widetilde{x}^{2},
$$

along with $\widetilde{y}, z, \widetilde{z}$. Let

$$
M_{\text {heat }, 2}^{2}:=\left[M_{\text {heat }, 1}^{2} ; \mathrm{ff}_{1} \cap \mathcal{B}_{1}\right]_{\text {inhom }},
$$

with $t^{\prime} \sim|\eta|^{2} \sim(s-1)^{2} \sim \widetilde{x}^{2(k-1)}$, so we have polar coordinates near ff given by

$$
\begin{aligned}
& \bar{\rho}=\left(\left(t / \widetilde{x}^{2}\right)+\widetilde{x}^{2(k-1)}+(s-1)^{2}+(|y-\widetilde{y}| / \widetilde{x})^{2}\right)^{1 / 2(k-1)}, \\
& \bar{\phi}:=\left(\bar{\phi}_{t}, \bar{\phi}_{\widetilde{x}}, \bar{\psi}_{x}, \bar{\psi}_{y}\right)=\left(\frac{t}{\widetilde{x}^{2} \bar{\rho}^{2(k-1)}}, \frac{\widetilde{x}}{\bar{\rho}}, \frac{x-\widetilde{x}}{\widetilde{x} \bar{\rho}^{(k-1)}}, \frac{y-\widetilde{y}}{\widetilde{x} \bar{\rho}^{(k-1)}}\right) \text { along with } \widetilde{y}, z, \widetilde{z} .
\end{aligned}
$$

Let

$$
\beta_{2}: M_{\text {heat }, 2}^{2} \longrightarrow M \times M \times[0, \infty)_{t}
$$

denote the blowdown map. Then, similar to the setup at $\mathrm{ff}_{1}$, if we define $\sigma=$ $(x-\widetilde{x}) / \widetilde{x}$, the interior of $\mathrm{ff}$ is a bundle over $Y \times \mathbb{R}_{\sigma}$ whose fiber over $p \in Y$ is isomorphic to $T_{p} Y \times Z^{2} \times \mathbb{R}_{\widetilde{T}}$ for $\widetilde{T}$ the rescaled time variable below.

See Remark 11 below for further discussion of the need for the two distinct blown up faces $\mathrm{ff}$ and $\mathrm{ff}_{1}$. 
(3) Finally, we blow up the time equals zero diagonal, $\mathcal{B}_{2}:=\operatorname{cl}\left(\beta_{2}\left(\operatorname{diag}\left(M^{\circ}\right) \times\{t=0\}\right)\right)$, where cl denotes the closure, parabolically in time. Note that $\mathcal{B}_{2}$ intersects the face ff at $\bar{\phi}=(1,0,0,0)$, so near the intersection, defining the functions

$$
\widetilde{x}, \quad \sigma=\frac{s-1}{\widetilde{x}^{k-1}}=\frac{x-\widetilde{x}}{\widetilde{x}^{k}}, \quad \widetilde{\eta}=\frac{y-\widetilde{y}}{\widetilde{x}^{k}}, \quad \widetilde{T}=\frac{t^{\prime}}{\widetilde{x}^{2(k-1)}}=\frac{t}{\widetilde{x}^{2 k}},
$$

we have the projective coordinates

$$
\widetilde{x}, \widetilde{y}, \sigma, \widetilde{\eta}, \widetilde{T}, z, \widetilde{z}
$$

The full heat space is

$$
M_{\text {heat }}^{2}=\left[M_{\text {heat }, 2}^{2} ; \mathcal{B}_{2}\right]_{\text {inhom }},
$$

with $\widetilde{T} \sim \sigma^{2} \sim(z-\widetilde{z})^{2}$. The face tf introduced by the final blowup satisfies

$$
\mathrm{tf}^{\circ} \simeq{ }^{i c e} T(M)
$$

where ${ }^{i c e} T(M)$ is the incomplete cusp edge tangent bundle defined in (2.8). Concretely, in coordinates $(x, y, z)$ if we set

$$
\xi=\frac{x-\widetilde{x}}{\sqrt{t}}, \quad \eta_{i}=\frac{y_{i}-\widetilde{y}_{i}}{\sqrt{t}}, \quad \zeta_{\alpha}=\frac{z_{\alpha}-\widetilde{z}_{\alpha}}{\sqrt{t}} \widetilde{x}^{k}, \quad \tau=\frac{\sqrt{t}}{\widetilde{x}^{k}},
$$

then $(x, y, z, \xi, \eta, \zeta, \tau)$ (or $(\widetilde{x}, \widetilde{y}, \widetilde{z}, \xi, \eta, \zeta, \tau)$ ) form local coordinates near the intersection of $\mathrm{tf}$ with $\mathrm{ff}$ and away from $t=0$, and the association $\xi \mapsto \partial_{x}, \eta_{i} \mapsto \partial_{y_{i}}, \zeta_{\alpha} \mapsto$ $x^{-k} \partial_{z_{\alpha}}$ induces the map.

In summary, we have constructed a manifold with corners $M_{\text {heat }}^{2}$, depicted in Figure 1 with a blowdown map $\beta$ as in 1.20 , such that $M_{\text {heat }}^{2}$ has six total faces, three of them being the lifts of the standard faces

$$
\text { If }:=\operatorname{cl}\left(\beta^{-1}\left(\{x=0\}^{\circ}\right)\right), \operatorname{rf}:=\operatorname{cl}\left(\beta^{-1}\left(\{\widetilde{x}=0\}^{\circ}\right)\right), \text { tb }:=\operatorname{cl}\left(\beta^{-1}\left(\{t=0\}^{\circ}\right)\right),
$$

and then the three faces $\mathrm{ff}_{1}, \mathrm{ff}$, and tf constructed (in that order) by radial blow up as described above. Denoting the set of the six boundary hypersurfaces by $\mathcal{M}\left(M_{\text {heat }}^{2}\right)=$ $\left\{\right.$ lf, $\left.\mathrm{rf}, \mathrm{tb}, \mathrm{ff}_{1}, \mathrm{ff}, \mathrm{tf}\right\}$, and given $\bullet \in \mathcal{M}\left(M_{\text {heat }}^{2}\right)$, below we will let $\rho_{\bullet}$ denote a boundary defining function for $\bullet$, so $\rho_{\bullet} \in C^{\infty}\left(M_{\text {heat }}^{2}\right)$ satisfies that $\left\{\rho_{\bullet}=0\right\}=\bullet$ and $d \rho_{\bullet}$ is non-vanishing on - and $\rho_{\bullet} \geq 0$. We can take $\rho_{\mathrm{ff}}=\bar{\rho}$ as in (3.7) and $\rho_{\mathrm{ff}_{1}}=\rho / \bar{\rho}$. Also note that

$$
x=\rho_{\mathrm{lf}} \rho=\rho_{\mathrm{lf}} \rho_{\mathrm{ff}} \rho_{\mathrm{ff}} .
$$

In Theorem 12 we will show that the heat kernel lifts to be polyhomogeneous on $M_{\text {heat }}^{2}$.

3.2. Model operators. The blown up space $M_{\text {heat }}^{2}$ is useful in the construction of a parametrix for the heat equation in part because the operator $\partial_{t}+\Delta$ (more specifically $\left.t\left(\partial_{t}+\Delta\right)\right)$ behaves nicely at the three introduced boundary hypersurfaces $\mathrm{ff}, \mathrm{ff}_{1}$, and $\mathrm{tf}$; in particular, the first steps in the parametrix construction involve finding the right asymptotic behavior for the heat kernel so that the heat equation (1.14) is satisfied at least to leading order at $\mathrm{ff}, \mathrm{ff}_{1}$, and $\mathrm{tf}$.

Thus, we consider the operator $\Delta$ acting on the left spacial factor of $M \times M \times[0, \infty)_{t}$, and the pullback $\beta^{*}\left(t\left(\partial_{t}+\Delta\right)\right)$ to $M_{\text {heat }}^{2}$, and show that this restricts to an operator at tf. To be precise, fix a point $p \in M$ and consider the fiber $\operatorname{tf}_{p}=\pi^{-1}(p)$ where $\pi:$ tf $\longrightarrow \operatorname{diag}_{M}=M$ is the projection onto the diagonal (or more concretely it is $\left.\beta\right|_{\mathrm{tf}}$ ). In the interior of tf, i.e. away from the intersection with ff, this is standard [31, so we concern ourselves only with an open neighborhood of the intersection of tf with ff. Indeed, working locally over the base 


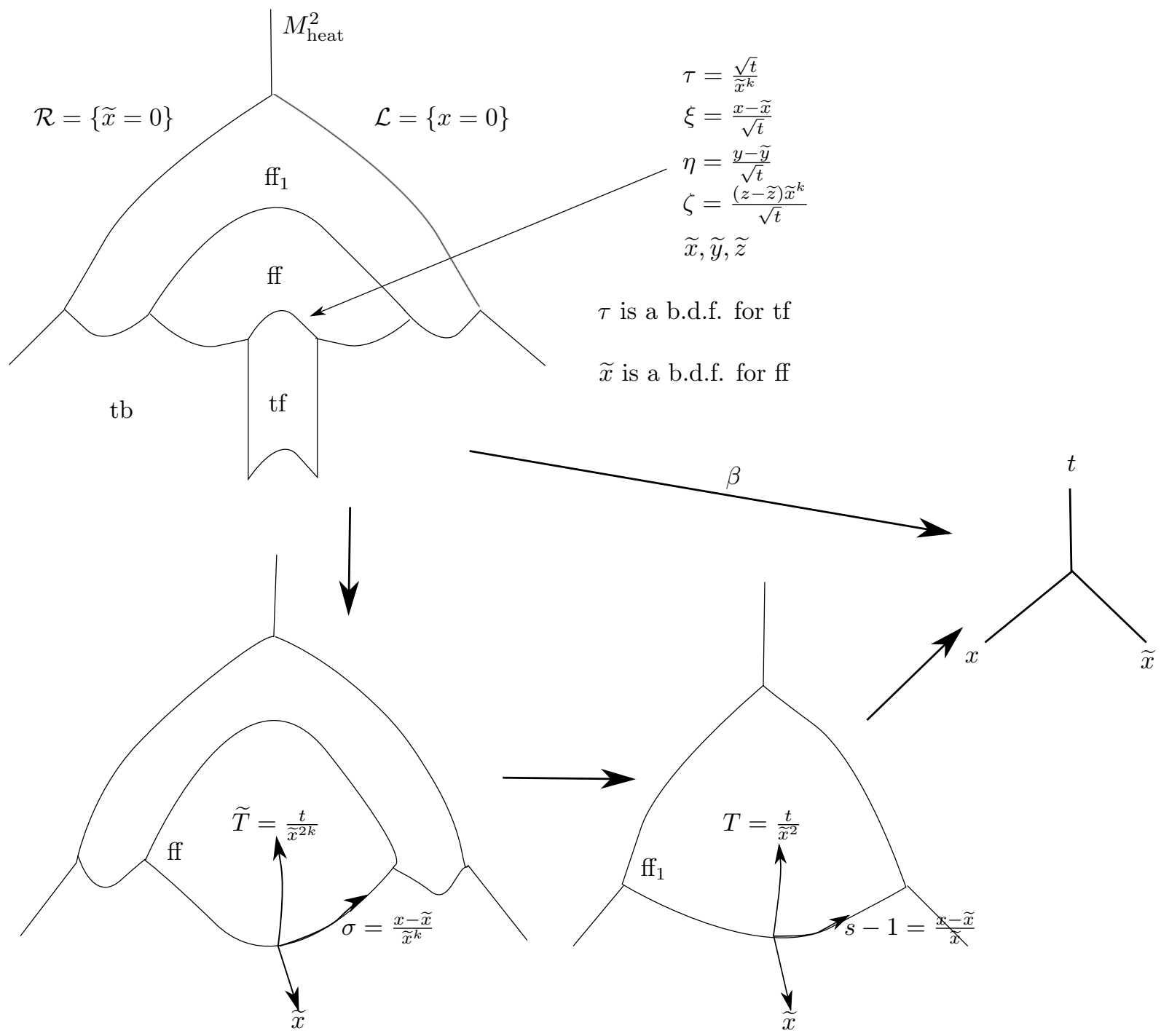

FiguRE 1. The heat double space (upper left) and the various intermediate blow ups together with their blow down maps.

in both spacial factors, consider a subset of tf of the form $\{(\widetilde{x}, \widetilde{y}, \widetilde{z}, \xi, \eta, \zeta, \tau):(\widetilde{x}, \widetilde{y}, \widetilde{z}) \in \mathcal{O}\}$. Now note

$$
\sqrt{t} \partial_{x}=\partial_{\xi}, \quad \sqrt{t} \partial_{y}=\partial_{\eta}, \quad \frac{\sqrt{t}}{x^{k}} \partial_{z}=\partial_{\zeta}+O(\tau)
$$

and

$$
t \partial_{t}=\frac{1}{2}\left(\tau \partial_{\tau}-R\right)
$$

where $R=\xi \partial_{\xi}+\eta \cdot \partial_{\eta}+\zeta \partial_{\zeta}$ is the radial vector field on the fiber.

Letting $\pi_{L}, \pi_{R}: M \times M \times[0, \infty)_{t} \longrightarrow M$ denote the projections onto the left and right $M$ factors, and End $\longrightarrow M \times M$ the endomorphism bundle, whose fiber at $(p, q) \in M^{\circ} \times M^{\circ}$ 
is $\operatorname{End}\left(\Lambda_{q}^{*} ; \Lambda_{p}^{*}\right)$, for $t>0$, the heat kernel restricted to the interior will be a smooth section of this bundle. To study the heat kernel at the boundary we use the incomplete cusp edge forms and the corresponding endomorphism bundle $\operatorname{End}\left({ }^{\text {ice }} \Lambda^{*}\right)$ back to $M \times M \times[0, \infty)_{t}$ and then to $M_{\text {heat }}^{2}$ via the blowdown $\beta$. As usual, restricting to the spacial diagonal gives the 'little endomorphism' bundle

$$
\left.\operatorname{End}\left({ }^{\text {ice }} \Lambda^{*}\right)\right|_{\operatorname{diag}(M)} \simeq \operatorname{end}\left({ }^{\text {ice }} \Lambda^{*}\right)
$$

where end $\left({ }^{\text {ice }} \Lambda^{*}\right) \longrightarrow M$ is the endomorphism bundle of the exterior algebra of $M$. The restriction to the time face, $\beta^{*}$ End $\left.\right|_{\text {tf }}$, is isomorphic to the pullback of end $\left(\Lambda_{p}^{*}\right)$ to the tangent bundle of $M$ via the projection map.

Writing $w=(x, y, z), \widetilde{w}=(\widetilde{x}, \widetilde{y}, \widetilde{z})$, sections of $\beta^{*}$ End near the fiber of tf over $p$ can be written

$$
\alpha=\sum_{I, J} a_{I J} d w_{I} \otimes \partial \widetilde{w}_{J}
$$

where $I, J$ run over all multi-indices and $\partial \widetilde{w}_{J}$ is dual to $d w_{J}$, and here $a_{I J}=a_{I J}(w, \widetilde{w}, t)$. We claim that, writing sections of $\beta^{*}$ End near tf as sections of $\beta^{*}$ End $\left.\right|_{\mathrm{tf}} \simeq \Lambda^{*}(M) \otimes \Lambda(M)$,

$$
t\left(\partial_{t}+\Delta\right)=\left(\frac{1}{2}\left(\tau \partial_{\tau}-R\right)+\sigma(\Delta)\right) \otimes \operatorname{Id}+O(\tau),
$$

where $\sigma(\Delta)$ is a constant coefficient differential operator in the coordinates $\Xi=(\xi, \eta, \zeta)$ depending on the metric $g$ at $p=(\widetilde{x}, \widetilde{y}, \widetilde{z})$, namely

$$
\sigma(\Delta)=\left(d_{\Xi}+\star_{g(p)}^{-1} d_{\Xi \star} \star_{g(p)}\right)^{2},
$$

acting on differential forms on the vector space ice $\Lambda_{p}^{*}(M)$ with metric $g(p)$. Indeed, let $w$ be geodesic normal coordinates. In the interior of tf away from $\mathrm{ff}$ we have coordinates $\Xi=(w-\widetilde{w}) / \sqrt{t}, \widetilde{w}, \sqrt{t}$. Then $t\left(\partial_{t}+\Delta\right) \alpha=\left(t\left(\partial_{t}+\Delta\right) \sum_{I, J} a_{I J} d w_{I}\right) \otimes \partial \widetilde{w}_{J}$ and moreover

$$
\begin{aligned}
\star d w_{I} \otimes \partial \widetilde{w}_{J} & =\left(\star d w_{I}\right) \otimes \partial \widetilde{w}_{J} \\
& = \pm d w_{I^{c}} \otimes \partial \widetilde{w}_{J}+O(w-\widetilde{w}) \\
& = \pm\left(d \widetilde{w}_{I^{c}}+\sqrt{t} d \Xi_{I^{c}}\right) \otimes \partial \widetilde{w}_{J}+O(\sqrt{t} \Xi) \\
& =\left(\star_{g(p)} d \widetilde{w}_{I}\right) \otimes \partial \widetilde{w}_{J}+O(\widetilde{t}),
\end{aligned}
$$

Similarly, letting the exterior derivative act on the left gives $d\left(a d w_{I} \otimes \partial \widetilde{w}_{J}\right)=\left(\partial_{\Xi_{i}} a d \widetilde{w}_{i} \wedge\right.$ $\left.\widetilde{w}_{I}\right) \otimes \partial \widetilde{w}_{J}$

To motivate our construction of the heat kernel further, in a neighborhood of tf let $\gamma$ be a section of End with the property that $\gamma \mid \operatorname{diag}_{M}=\mathrm{Id}$ on the form bundles, and consider the section of $\beta^{*}$ End on $M_{\text {heat }}^{2}$ of the form

$$
K(p, q, t)=\frac{1}{(2 \pi t)^{n / 2}} e^{-G(p, q) / 2 t} \gamma,
$$

such that $G(p, q)$ satisfies that $\left.\beta^{*}(G(p, q) / t)\right|_{\mathrm{tf}}=\|\Xi\|_{g}^{2}$, that is, that $G(p, q) / t$ restricts to the metric function on tf. Such a form $\gamma$ and function $G$ can be constructed but we neither prove nor use this; we merely use it as motivation. It is straightforward to check that for any smooth compactly supported form $\alpha$

$$
\lim _{t \rightarrow 0} \int_{M} K(p, q, t) \alpha(q) \mathrm{dVol}_{q}=(4 \pi)^{-n / 2} \int_{M} e^{-\|\Xi\|_{g(p)}^{2} / 4} \alpha(p) \sqrt{g(p)}|d \Xi|=\alpha(p),
$$

and in fact the convergence takes place in $L^{2}$. (In fact, such an endomorphism $\gamma$ can be constructed easily by taking the identity map on ${ }^{\text {ice }} \Lambda^{*}$ over $M$, pulling this back via $\beta$ to 
$\beta^{*}$ End $\left.\right|_{\mathrm{tf}}$ and extending off smoothly in a neighborhood. On each exterior algebra $\Lambda_{p}^{*} M$, the identity can be expressed in terms of a basis $e_{i}$ with dual basis $e_{i}^{*}$ as $\sum_{I} e_{I} \otimes e_{I}^{*}$. In a neighborhood of $\mathrm{tf} \cap \mathrm{ff}$ we can take the basis $e_{i}$ to be $d x, d y_{i}, x^{k} d z_{\alpha}$, i.e. we can take the $e_{i}$ to be a basis of forms for ${ }^{\text {ice }} \Lambda^{*}$ all the way down to $x=0$.)

Working in the coordinates (3.13), since $t^{-n / 2}=\tau^{-n} \widetilde{x}^{-n k}$, the Taylor expansion of the heat kernel at tf should take the form

$$
\frac{1}{(4 \pi)^{n / 2} \widetilde{x}^{k n}} \tau^{-n} \sum_{j=0}^{\infty} \tau^{j} b_{j}
$$

where the $b_{j}=b_{j}(\widetilde{x}, \widetilde{y}, \widetilde{z}, \xi, \eta, \zeta)$ are sections of $\beta^{*}$ End, which we again write in a neighborhood of $\mathrm{tf} \cap \mathrm{ff}$ as sections of $\operatorname{End}\left({ }^{(i c e} \Lambda^{*}\right)$ pulled back to the fibers of tf. Writing each $b_{j}$ as a finite sum of terms of the form

$$
\alpha \otimes g^{-1} \beta,
$$

where $\alpha$ and $\beta$ are sections of ice $\Lambda^{*}$ and $g^{-1}$ indicates taking the dual vector field, we see that by $(3.19)$ we have,

$$
\left(\left(\frac{n}{2}-\frac{1}{2} R+\sigma(\Delta)\right) \otimes \mathrm{Id}\right) b_{0}=\left(\left(\frac{n}{2}-\frac{1}{2} R+\left(\begin{array}{cc}
\Delta_{\Xi} & 0 \\
0 & \Delta_{\Xi}
\end{array}\right)\right) \otimes \mathrm{Id}\right) b_{0} .
$$

The only solution to this equation which gives the identity operator at $t=0$ is

$$
b_{0}=e^{-\|\Xi\|^{2} / 4} \times \operatorname{Id} .
$$

The procedure of solving for the remaining $b_{j}$ is standard [31, Chapter 7]; letting the Laplacian act on this expansion we show that on each term $a_{j}$ it acts fiberwise like a constant coefficient, second order elliptic differential operator plus the radial vector field plus a constant corresponding to the order of the term in the expansion. We have the following

Lemma 8. There exist sections $b_{j}$ of $\mathcal{A}_{\mathrm{phg}}\left(\left.\mathrm{End}\right|_{\mathrm{tf}}\right)$ satisfying

$$
b_{j}=e^{-\|\Xi\|^{2} / 4} \widetilde{b}_{j}(\widetilde{x}, \widetilde{y}, \widetilde{z}, \xi, \eta, \zeta)
$$

where $\widetilde{b}_{j}$ is a polynomial in $\xi, \eta, \zeta$ and a polyhomogeneous section of End over $\mathrm{tf}$, such that for any distribution $H^{\prime}$ in $\mathcal{A}^{\mathrm{phg}}$ (End) with asymptotic expansion near tf given by (3.24) we have

$$
t\left(\partial_{t}+\Delta\right) H^{\prime}=O\left(\tau^{\infty}\right),
$$

i.e. $t\left(\partial_{t}+\Delta\right) H^{\prime}$ vanishes to infinite order at the blown up $t=0$ diagonal, and, moreover, the asymptotic sum of the $b_{j}$ exists and yields such an $H^{\prime}$.

The existence of a distribution $H^{\prime}$ as in Lemma 8 is only a first step in constructing a parametrix for the heat kernel. We will discuss the rest of the process in $\$ 3.3$.

A useful double check of the order of blow up of the heat kernel at $\mathrm{ff}$ is the following. Near $\mathrm{ff} \cap \mathrm{tf}$ we have

$$
\begin{aligned}
\delta(x-\widetilde{x}) \delta(z-\widetilde{z}) \delta(y-\widetilde{y}) & =\delta\left(\xi \tau \widetilde{x}^{k}\right) \delta\left(\eta \tau \widetilde{x}^{k}\right) \delta(\zeta \tau) \\
& =\frac{1}{\tau^{n} \widetilde{x}^{(n-f) k}} \delta(\xi) \delta(\eta) \delta(\zeta) .
\end{aligned}
$$

Since Id $=\lim _{t \searrow 0} H \mathrm{dVol}_{g} \sim \lim _{t \searrow 0} H \widetilde{x}^{k f} d \widetilde{x} d \widetilde{y} d \widetilde{z}$, we confirm that $H$ should have order $-n k$ at $\mathrm{ff}$. In fact, we can deduce more; considering $\left.\widetilde{x}^{k n} H\right|_{\mathrm{ff}}$, on the interior of ff we can use coordinates in $(3.10)$, we get that

$$
\delta(x-\widetilde{x}) \delta(y-\widetilde{y}) \delta(z-\widetilde{z})=\widetilde{x}^{-(n-f) k} \delta(\sigma) \delta(\widetilde{\eta}) \delta(z-\widetilde{z}),
$$


which means that, on the face ff, we expect that the restriction $\left.\widetilde{x}^{n k} H\right|_{\mathrm{ff}}$ will be given by $\delta(\sigma) \delta(\widetilde{\eta}) \delta(z-\widetilde{z})$ at least as the time variable $\widetilde{T}=t / \widetilde{x}^{k}$ goes to zero, as that is the region in which the action of $H$ is definitively approximated by the identity. On the other hand, $\widetilde{x}$ commutes with the heat operator $\partial_{t}+\Delta$. As we will see in (3.31), $t\left(\partial_{t}+\Delta\right)$ restricts to an operator on ff and defines a fiber-wise heat type operator on ff, so we expect to have

$$
\left.\left.t\left(\partial_{t}+\Delta\right)\right|_{\mathrm{ff}}\left(\widetilde{x}^{n k} H\right)\right|_{\mathrm{ff}}=0 .
$$

This, together with (3.28), implies that an ansatz for the heat kernel should include that on each fiber of $\mathrm{ff},\left.\widetilde{x}^{n k} H\right|_{\mathrm{ff}}$ is the fundamental solution to the induced heat equation on the fiber, more precisely, it is the solution which equals $\delta_{\sigma=0} \delta_{\tilde{\eta}=0} \operatorname{Id} z$ at time equals zero. The induced heat equations are translation invariant in $\sigma$ and $\widetilde{\eta}$, thus induced by convolution operators, and the heat kernels we speak of are the convolution kernels in $\sigma$ and $\widetilde{\eta}$.

As for the blow up at $\mathrm{ff}_{1}$, as we will see below, the operator acts as a modified heat operator in $\partial_{x}$ and $Y$ on the bundle of fiber harmonic forms, so in the coordinates in 3.5 we will have

$$
\delta(x-\widetilde{x}) \delta(y-\widetilde{y}) \delta(z-\widetilde{z})=\frac{1}{\widetilde{x}^{1+b}} \delta(s-1) \delta(z-\widetilde{z}) \delta(\eta) .
$$

In this case, $t\left(\partial_{t}+\Delta\right)$ only admits a restriction to $\mathrm{ff}_{1}$ on the fiber-harmonic forms $\mathcal{H}$, on which $\delta(z-\widetilde{z})$ becomes projections $\Pi_{Z, y}$ onto the kernel of $\Delta^{Z, y}$. Thus we expect that $\left.\widetilde{x}^{1+b+k f} H\right|_{\mathrm{ff}_{1}}$ on fiber harmonic forms is given by the convolution kernel for the heat kernel in $\eta$, times the dilation invariant kernel for the heat kernel in $s$ with limit $\delta_{s=1}$ at time 0.

We now compute the asymptotic behavior of $t\left(\partial_{t}+\Delta\right)$ at the faces $\mathrm{ff}$ and $\mathrm{ff}_{1}$. First we will work at ff.

Proposition 9 (The model problem on ff). The operator $N_{\mathrm{ff}}\left(t\left(\partial_{t}+\Delta^{g}\right)\right)=\left.t\left(\partial_{t}+\Delta^{g}\right)\right|_{\mathrm{ff}}$ acts fiberwise on $\mathrm{ff}$, and is expressed in the coordinates in 3.10 by

$$
N_{\mathrm{ff}}\left(t\left(\partial_{t}+\Delta^{g}\right)\right)=\widetilde{T}\left(\partial_{\widetilde{T}}+\left(\begin{array}{cc}
-\partial_{\sigma}^{2}+\Delta_{\eta}+\Delta^{Z, y} & 0 \\
0 & -\partial_{\sigma}^{2}+\Delta_{\eta}+\Delta^{Z, y}
\end{array}\right)\right)
$$

on the fiber above $y \in Y$. Here $\Delta_{\eta}$ is the constant coefficient Hodge-Laplacian on the tangent space $T_{y} Y$ with translation invariant metric $h(y)$, and $\Delta^{Z, y}$ is the Hodge-Laplacian on $\left(Z, \boldsymbol{k}_{y}\right)$

The situation is more delicate at $\mathrm{ff}_{1}$. As we will see in Section 3.3 near $\mathrm{ff}_{1}$, it will suffice to consider $t\left(\partial_{t}+\Delta\right)$ restricted to approximately fiber harmonic forms, (see Section 2.3). Thus let $\gamma \in x^{s} \mathcal{H}_{\mathrm{phg}}\left({ }^{\mathrm{ice}} \Lambda^{*}\right)$ with the space on the right defined in $(2.28)$, and thus by assumption $\delta^{Z} \gamma, d^{Z} \gamma$ are both $O\left(x^{s+k}\right)$. From 2.15 it follows that for such fiber harmonic forms,

$$
\Delta \gamma=\widetilde{\Delta}_{0} \gamma+x^{-2 k} \Delta_{Z} \gamma+x^{-k}\left(\delta_{Z} Q_{1}+d_{Z} Q_{2}\right) \gamma+O\left(x^{s-1}\right)
$$

where $\widetilde{\Delta}_{0}$ acts on forms decomposed as in 2.6 as

$$
\widetilde{\Delta}_{0}=-\partial_{x}^{2}-\frac{k f}{x} \partial_{x}+\Delta_{Y}+\left(\begin{array}{cc}
k \mathbf{N}(1-k(f-\mathbf{N})) x^{-2} & -2 k x^{-k-1} d^{Z} \\
-2 k x^{-k-1} \delta^{Z} & k(f-\mathbf{N})(1-k \mathbf{N}) x^{-2}
\end{array}\right) .
$$

By fiber harmonicity, $x^{-2 k} \Delta_{Z} \gamma=O\left(x^{s-k}\right)$. Thus the two terms $x^{-2 k} \Delta_{Z}$ and $x^{-k} \delta_{Z} Q_{1}$ act on fiber harmonic forms as operators of order $x^{-k}$, and thus in the heat operator $t\left(\partial_{t}+\Delta\right)$ there are term behaving like $t x^{-k}$ (on fiber harmonic forms) and $t / x^{-k}$ is not a bounded function at $\mathrm{ff}_{1}$ ! On the other hand, if we project back to the fiber harmonic forms, then by 
2.31- 2.32 we kill these terms; concretely, with $\Pi_{\mathcal{H}}$ the fiber harmonic projector in 2.30, we have

$$
\Pi_{\mathcal{H}} \Delta \Pi_{\mathcal{H}}=\widetilde{\Delta}_{0}+x^{-1} \widetilde{E}^{\prime}
$$

where $\widetilde{E}^{\prime} \in \operatorname{Diff}_{\mathrm{b}, \mathrm{phg}}^{2}$ (see 2.13 ), and thus does not decrease the order of vanishing of polyhomogeneous distributions. Defining

$$
P_{A, B}:=-\partial_{s}^{2}-\frac{A}{s} \partial_{s}+\frac{B}{s^{2}} .
$$

and

$$
\alpha(\mathbf{N}):=k f, \quad \beta(\mathbf{N}):=k \mathbf{N}(1-k(f-\mathbf{N})), \quad \gamma(\mathbf{N})=k(f-\mathbf{N})(1-k \mathbf{N}),
$$

we have the following.

Proposition 10 (Heat operator on fiber harmonic forms at $\mathrm{ff}_{1}$ ). Restricted to the fiber harmonic forms $\mathcal{H}$ as defined through 2.25,

$$
N_{\mathrm{ff}_{1}}\left(t\left(\partial_{t}+\Delta^{g}\right)\right):=\left.\Pi_{\mathcal{H}} t\left(\partial_{t}+\Delta\right) \Pi_{\mathcal{H}}\right|_{\mathrm{ff}_{1}}
$$

restricts to the face $\mathrm{ff}_{1}$ in the coordinates 3.5 as

$$
N_{\mathrm{ff}_{1}}\left(t\left(\partial_{t}+\Delta^{g}\right)\right)=t^{\prime}\left(\partial_{t^{\prime}}+\left(\begin{array}{cc}
P_{\alpha(\mathbf{N}), \beta(\mathbf{N})}+\Delta_{\eta} & 0 \\
0 & P_{\alpha(\mathbf{N}), \gamma(\mathbf{N})}+\Delta_{\eta}
\end{array}\right)\right) .
$$

Remark 11. Analysis of the fiber harmonic forms is necessary in particular because the structure of the operator $\Delta^{g}$ is such that, off of the fiber harmonic forms, the leading order term is $x^{-2 k} \Delta^{Z, y}$, while restricted to the fiber harmonic foms the leading order term drops in order. Indeed, if it weren't for the presence of the term $x^{-k} \widetilde{P}$ in 2.15 , which presents complications in the analysis, on fiber harmonic forms $\Delta^{g}$ would be given by to leading order by $\widetilde{\Delta}_{0}$. Indeed, the need for the two different regimes represented by the boundary hypersurfaces $\mathrm{ff}$ and $\mathrm{ff}_{1}$ is exactly this change in asymptotic order of the operator on and off the fiber harmonic forms. Correspondingly, we will see below in the proof of Lemma 14 that the operator $t\left(\partial_{t}+\Delta\right)$ restricted to $\mathrm{ff}$ has a fundamental solution which vanishes at $\mathrm{ff}_{1}$ to infinite order off the fiber harmonic forms.

The heat equation for the regular singular ODEs in (3.34) has been studied in detail. To such an operator there corresponds a pair of indicial roots given by the order of vanishing of homogeneous solutions, specifically $P_{A, B}\left(s^{\ell}\right)=0$ if and only if

$$
\ell=\frac{-(A-1) \pm \sqrt{(A-1)^{2}+4 B}}{2} .
$$

The numbers $\ell$ give important information about the operator $P_{A, B}$, in particular they give the order of vanishing of the Green's function at $s=0$. The operators that will arise in our work are those in the matrices in (3.37). We define the indicial set

$$
\begin{aligned}
\Lambda & =\bigcup_{\mathbf{N}=1}^{f}\left\{\frac{-(\alpha-1) \pm \sqrt{(\alpha-1)^{2}+4 \beta}}{2}, \frac{-(\alpha-1) \pm \sqrt{(\alpha-1)^{2}+4 \gamma}}{2}\right\} \\
& =\bigcup_{\mathbf{N}=1}^{f}\{-(k f-1) / 2 \pm|k(\mathbf{N}-f / 2)+1 / 2|,-(k f-1) / 2 \pm|k(\mathbf{N}-f / 2)-1 / 2|\}
\end{aligned}
$$

Letting

$$
\nu^{2}=B+\left(\frac{A-1}{2}\right)^{2}>0
$$


where $\nu>0$, from [35, Vol. 2, Eqn. 8.45] there is a fundamental solution $H_{A, B}(s, \widetilde{s}, t)$

$$
\left(\partial_{t}+P_{A, B}\right) H_{A, B}(s, \widetilde{s}, t)=0 \text {, and } H \rightarrow \operatorname{Id} \text { as } t \rightarrow 0 \text {, on } L^{2}\left(s^{A} d s\right) .
$$

Indeed, one has the explicit formula

$$
H_{A, B}(s, \widetilde{s}, t)=(s \widetilde{s})^{-(A-1) / 2} \frac{1}{2 t} e^{-\left(s^{2}+\widetilde{s}^{2}\right) / 4 t} I_{\nu}\left(\frac{s \widetilde{s}}{2 t}\right)
$$

where $I_{\nu}$ is the modified Bessel function of order $\nu$ of the first kind [1, Chap. 9].

As discussed below (3.30), at the face $\mathrm{ff}_{1}$ we expect the heat kernel to be of order $\widetilde{x}^{-1-b-k f}$. Thus we expect to have

$$
0=t\left(\partial_{t}+\Delta\right) H=\frac{1}{\widetilde{x}^{1+b+k f}}\left(t\left(\partial_{t}+\Delta\right)\right)\left(\widetilde{x}^{1+b+k f} H\right),
$$

and since $\Pi_{\mathcal{H}} t\left(\partial_{t}+\Delta\right) \Pi_{\mathcal{H}}$ defines a differential operator on section of $\mathcal{H} \otimes \overline{\mathcal{H}}^{*}$ restricted to $\mathrm{ff}_{1}$, we include in our ansatz for the fundamental solution (1.14), and indeed prove in Theorem 12 below, that there is a fundamental solution $H$ satisfying that $\widetilde{x}^{1+b+k f} H$ has a smooth restriction to $\mathrm{ff}_{1}$, and writing

$$
N_{\mathrm{ff}_{1}}(H):=\left.\left(\widetilde{x}^{1+b+k f} H\right)\right|_{\mathrm{ff}_{1}} \text {, we have } N_{\mathrm{ff}_{1}}\left(t\left(\partial_{t}+\Delta\right)\right) N_{\mathrm{ff}_{1}}(H)=0 .
$$

Furthermore, again as discussed below (3.30), it is sensible to include in the ansatz for $H$ that $N_{\mathrm{ff}_{1}}(H)$ is the fundamental solution for the model operator $N_{\mathrm{ff}_{1}}\left(t\left(\partial_{t}+\Delta\right)\right)$, meaning specifically that $N_{\mathrm{ff}_{1}}(H)$ is a section of the restriction of the sub-bundle $\operatorname{End}(\mathcal{H})$ to $\mathrm{ff}_{1}$ and is given using the fundamental solutions to the model heat equations $H_{A, B}$ from (3.41)-(3.42). Specifically, we will have as an ansatz that $N_{\mathrm{ff}_{1}}(H)=\kappa_{\mathrm{ff}_{1}}$, where

$$
\kappa_{\mathrm{ff}_{1}, y}:=\left(\begin{array}{cc}
H_{\alpha, \beta}\left(s, 1, t^{\prime}\right) & 0 \\
0 & H_{\alpha, \gamma}\left(s, 1, t^{\prime}\right)
\end{array}\right)\left(4 \pi t^{\prime}\right)^{-b / 2} e^{-|\eta|_{y}^{2} / 4 t^{\prime}},
$$

where $\alpha, \beta, \gamma$ are as in (3.35), and in particular continue to be operators depending on the fiber form degree $\mathbf{N}$. The distribution $N_{\mathrm{ff}_{1}}(H)$ is polyhomogeneous on $\mathrm{ff}_{1}$, and the leading order behavior at $s=0$ satisfies that for $0<c \leq t^{\prime} \leq C<\infty$, for some smooth $a\left(t^{\prime}\right), b\left(t^{\prime}\right)$,

$$
H_{\alpha, \beta}\left(s, 1, t^{\prime}\right) \sim s^{-(k f-1) / 2} a\left(t^{\prime}\right) s^{\nu(\alpha, \beta)}, \quad H_{\alpha, \gamma}\left(s, 1, t^{\prime}\right) \sim s^{-(k f-1) / 2} b\left(t^{\prime}\right) s^{\nu(\alpha, \gamma)}
$$

with $\nu$ as in 3.40

$$
\begin{aligned}
& \nu(\alpha, \beta)= \begin{cases}k(f / 2-\mathbf{N})-1 / 2 & \text { if } \mathbf{N}<f / 2, \\
k(\mathbf{N}-f / 2)+1 / 2 & \text { if } \mathbf{N} \geq f / 2,\end{cases} \\
& \nu(\alpha, \gamma)= \begin{cases}k(f / 2-\mathbf{N})+1 / 2 & \text { if } \mathbf{N} \leq f / 2, \\
k(\mathbf{N}-f / 2)-1 / 2 & \text { if } \mathbf{N}>f / 2,\end{cases}
\end{aligned}
$$

and thus by (3.46) on $\mathrm{ff}_{1}$ in the region $0<c \leq t^{\prime} \leq C<\infty$,

$$
\kappa_{\mathrm{ff}_{1}}=O\left(s^{\bar{\nu}}\right) \text { where } \bar{\nu}(\mathbf{N})=\left\{\begin{array}{cc}
-k \mathbf{N} & \text { if } \mathbf{N}<f / 2, \\
-k \mathbf{N}+1 & \text { if } \mathbf{N}=f / 2, \\
-k(f-\mathbf{N}) & \text { if } \mathbf{N}>f / 2 .
\end{array}\right.
$$

In words, each $P_{\alpha, \beta}$ has two indicial roots, the order of $H_{\alpha, \beta}$ for fixed $\widetilde{s}, t>0$ is the larger of these two, and $p$ is the smaller of the leading orders of $H_{\alpha, \beta}$ and $H_{\alpha, \gamma}$.

The behavior of the heat kernel at $\mathrm{ff}_{1}$ also shows what to expect at the left face, the lift of $x=0$. There we should just have the projection onto the fiber harmonic forms times the 
leading order behavior of the $H_{\alpha, \beta}$ and $H_{\alpha, \gamma}$, acting appropriately on ${ }^{\text {ice }} \Lambda^{*}$, times the lifted heat kernel of the base $Y$. Indeed, we expect

$$
\Pi_{\mathcal{H}} H \Pi_{\mathcal{H}} \simeq \kappa:=\left(\begin{array}{cc}
H_{\alpha(\mathbf{N}), \beta(\mathbf{N})}(x, \widetilde{x}, t) & 0 \\
0 & H_{\alpha(\mathbf{N}), \gamma(\mathbf{N})}(x, \widetilde{x}, t)
\end{array}\right) H_{Y}
$$

where $H_{Y}$ is the heat kernel on $(Y, h)$ lifted to the tubular neighborhood $\mathcal{U}$ in (2.1) via the projection and $\kappa$ acts on sections of the bundle of fiber harmonic forms $\mathcal{H}$ with its grading by fiber form degree $\mathbf{N}$. (See Section 2.3 ) In fact, with $\bar{\nu}=\bar{\nu}(\mathbf{N})$ the fiber degree dependent weight in (3.48),

$$
\kappa \in x^{\widetilde{\nu}(\mathbf{N})} C^{\infty}\left(M \times M ; \oplus_{\mathbf{N}=0}^{f} \mathcal{H}^{\mathbf{N}} \otimes\left(\widetilde{\mathcal{H}}^{\mathbf{N}}\right)^{*}\right)
$$

where $\mathcal{H}$ and $\widetilde{\mathcal{H}}$, respectively, the pullbacks of the fiber harmonic form bundle (defined on a neighborhood $\mathcal{U}$ of the boundary) via the left and right projections of $M \times M$.

As discussed below (3.29), on the face ff, we expect that the heat kernel will have leading asymptotic $\widetilde{x}^{-n k}$, so we expect and prove that

$$
N_{\mathrm{ff}}(H):=\left.\left(\widetilde{x}^{n k} H\right)\right|_{\mathrm{ff}}, \Longrightarrow N_{\mathrm{ff}}\left(t\left(\partial_{t}+\Delta\right)\right) N_{\mathrm{ff}}(H)=0 .
$$

Again, we will set $N_{\mathrm{ff}}(H)$ equal to a fundamental solution to the heat equation, namely, using the decomposition in (3.37), we expect to have $N_{\mathrm{ff}}(H)=\kappa_{\mathrm{ff}}$ where

$$
\kappa_{\mathrm{ff}, y}\left(\sigma, \eta, z, z^{\prime}, \widetilde{T}\right):=\operatorname{Id}_{2 \times 2}(4 \pi \widetilde{T})^{-(b+1) / 2} e^{-\left(\sigma^{2}+|\eta|_{h_{1}}^{2}\right) / 4 \widetilde{T}} H_{Z, y},
$$

where $H_{Z, y}=H_{Z, y}\left(z, z^{\prime}, \widetilde{T}\right)$ is the heat kernel for $\Delta^{Z, y}$.

Before stating the full structure theorem for the heat kernel let us briefly recall the notion of an index set, which by definition is a set of exponents $\mathcal{E}(\bullet)=\{(\gamma, p)\} \subset \mathbb{C} \times \mathbb{N}$ associated with each face $\bullet \in\left\{\right.$ lf, rf, tb, $\left.\mathrm{ff}_{1}, \mathrm{ff}, \mathrm{tf}\right\}$ such that

(i) each half-plane $\operatorname{Re} \gamma<C$ contains only finitely many $\gamma$;

(ii) for each $\gamma$, there is a number $P(\gamma) \in \mathbb{N}_{0}$ such that $(\gamma, p) \in \mathcal{E}(\bullet)$ for every $0 \leq p \leq$ $P(\gamma)$ and $(\gamma, p) \notin \mathcal{E}(\bullet)$ if $p>P(\gamma)$

(iii) If $(\gamma, p) \in \mathcal{E}(\bullet)$, then $(\gamma+j, p) \in \mathcal{E}(\bullet)$ for all $j \in \mathbb{N}$.

We give a full rigorous definition of polyhomogeneity in Section A, but roughly speaking, we call a differential form $\alpha$ polyhomogeneous with index family $\mathcal{E}=\{\mathcal{E}(\bullet) \mid \bullet \in$ $\left.\left\{\mathrm{lf}, \mathrm{rf}, \mathrm{tb}, \mathrm{ff}_{1}, \mathrm{ff}, \mathrm{tf}\right\}\right\}$ if it has an expansion at each boundary hypersurface $\bullet$ with exponents determined by the corresponding index set $\mathcal{E}(\bullet)$ and coefficient functions which are themselves polyhomogeneous (with exponents determined by $\mathcal{E}$ ). For example, smooth functions on $M_{\text {heat }}^{2}$ are polyhomogeneous with indicial set satisfying $\mathcal{E}(\bullet)=\mathbb{Z} \times\{0\}$ for all $\bullet$, and if a polyhomogeneous function vanishes to infinite order at a particular boundary hypersurface $\bullet$, then it is polyhomogeneous with an index set $\mathcal{E}$ satisfying $\mathcal{E}(\bullet)=\varnothing$. We define

$$
\inf \mathcal{E}(\bullet)=\inf \{\gamma \mid(\gamma, p) \in \mathcal{E}(\bullet)\} .
$$

Theorem 12. There exists a section $H$ of $\beta^{*}$ End over $M_{\text {heat }}^{2}$ which is polyhomogeneous, i.e. $H \in \mathcal{A}_{\mathrm{phg}}^{\mathcal{E}}\left(M_{\text {heat }}^{2} ; \beta^{*}\right.$ End) for some index set $\mathcal{E}$ and satisfying the following properties.

(1) In the interior of $M_{\text {heat }}^{2},\left(\partial_{t}+\Delta\right) H=0$.

(2) The operator $H_{t}$ defined initially on forms $\alpha \in C_{c}^{\infty}\left(M ; \Omega^{*}(M)\right)$ by

$$
H_{t} \alpha(w)=\int_{M} H(w, \widetilde{w}, t) \alpha(\widetilde{w}) \mathrm{dVol}_{\widetilde{w}}
$$

is symmetric on $L^{2}\left(d \mathrm{Vol}_{g}\right)$, and for such $\alpha$

$$
H_{t} \alpha \rightarrow \alpha \text { as } t \rightarrow 0
$$


in $L^{2}$.

(3) The index set $\mathcal{E}$ of $H$ satisfies that $\mathcal{E}(\mathrm{tb})=\varnothing$, while

$$
\inf \mathcal{E}\left(\mathrm{ff}_{1}\right) \geq-1-b-k f, \quad \inf \mathcal{E}(\mathrm{ff}) \geq-k n, \quad \mathcal{E}(\mathrm{tf})=\mathbb{N}-\operatorname{dim}(M),
$$

where $\mathcal{E}(\bullet) \geq c$ means that $\inf _{\zeta, p \in \mathcal{E}(\bullet)} \operatorname{Re} \zeta \geq c$.

(4) Moreover, at the faces $\mathrm{ff}$ and $\mathrm{ff}_{1}$,

$$
\left.\left(\widetilde{x}^{1+b+k f} H\right)\right|_{\mathrm{ff}_{1}}=\kappa_{\mathrm{ff}_{1}},\left.\quad\left(\widetilde{x}^{k n} H\right)\right|_{\mathrm{ff}}=\kappa_{\mathrm{ff}},
$$

with $\kappa_{\mathrm{ff}_{1}}$ and $\kappa_{\mathrm{ff}}$ the model heat kernels defined in (3.45) and (3.52).

(5) At the left face lf, with $\kappa$ as in 3.49)

$$
H_{t}=\kappa\left(1+O\left(\rho_{\mathrm{lf}}^{k}\right)\right) \text {. }
$$

In particular, for $t>0$ fixed, $H_{t}$ is approximately fiber harmonic in the sense of (2.25), and $\rho_{\mathrm{lf}}$ is a boundary defining function for lf (see (3.15)). Thus

$$
\inf \mathcal{E}(\text { lf }) \geq-\frac{k f}{2}+1 \text {. }
$$

The behavior of $H_{t}$ at the right face rf can be deduced from symmetry. Moreover for the behavior at the codimension 2 face lf $\cap \mathrm{rf}$, the leading order behavior is the product of that at lf and $\mathrm{rf}$, i.e. $H_{t}=O\left(\left(\rho_{\mathrm{lf}} \rho_{\mathrm{rf}}\right)^{-k f / 2+1}\right)$.

The proof of Theorem 12 is at the end of 3.3 .

3.3. Parametrix construction. We will establish the following

Proposition 13. There exists a polyhomogeneous section

$$
K \in \mathcal{A}_{\text {phg }}\left(M_{\text {heat }}^{2} ; \beta^{*}(\text { End })\right)
$$

such that $K$ satisfies (3.54)-(3.57) above, and such that $Q:=t\left(\partial_{t}+\Delta\right) K$, which is polyhomogeneous, has index set $\mathcal{E}^{\prime}$ satisfying

$$
\mathcal{E}^{\prime}\left(\mathrm{ff}_{1}\right) \geq-1-b-k f+1, \quad \mathcal{E}^{\prime}(\mathrm{ff}) \geq-k n+1, \quad \mathcal{E}^{\prime}(\mathrm{lf})=\mathcal{E}^{\prime}(\mathrm{tf})=\mathcal{E}^{\prime}(\mathrm{tb})=\varnothing .
$$

Our work above allows us to break the proof of the proposition into two main steps; first we will prove the following lemma:

Lemma 14. There exists a polyhomogeneous $K_{1}$ satisfying (3.54)-(3.57) above, together with (3.24) for the indicated $b_{j}$. Furthermore, $K_{1}$ can be taken fiber harmonic in a neighborhood of $\mathrm{ff}_{1}$. In fact we can assume that $K_{1} \equiv \kappa$ in a neighborhood of If and also in a neighborhood of $\mathrm{rf}$, where $\kappa$ is as in (3.57).

Proof of Proposition 13 assuming Lemma 14. Assuming that we have such a distribution $K_{1}$, we study $t\left(\partial_{t}+\Delta\right) K_{1}$. Automatically we have that $t\left(\partial_{t}+\Delta\right) K_{1}$ vanishes to infinite order at tf and tb, as follows from Lemma 8. Furthermore, $t\left(\partial_{t}+\Delta\right) K_{1}$ vanishes to order $-k n+1$ at ff by (3.31) and the fact that the leading order term $\kappa_{\mathrm{ff}}$ there solves the model problem.

At $\mathrm{ff}_{1}$ things are again more delicate. Recall that $K_{1}=O\left(\rho_{\mathrm{ff}}^{-1-b-k f}\right)$ at $\mathrm{ff}_{1}$, where $\rho_{\mathrm{ff}_{1}}$ is the boundary defining function for $\mathrm{ff}_{1}$ in, e.g. $\rho_{\mathrm{ff}_{1}}=\rho / \bar{\rho}$ with $\rho$ as in (3.3) and $\bar{\rho}$ as in (3.7). Since $K_{1}$ is fiber harmonic near $\mathrm{ff}_{1}$, by 2.30 and 3.32 we have

$$
\begin{aligned}
\Delta K_{1}= & \widetilde{\Delta}_{0} K_{1}+x^{-2 k} \Delta^{Z, y} K_{1}+x^{-k}\left(\delta_{Z} Q_{1}+d_{Z} Q_{2}\right) K_{1}+x^{-1} \widetilde{E} K_{1} \\
= & \widetilde{\Delta}_{0} K_{1}+x^{-k}\left(\delta_{Z} Q_{1}+d_{Z} Q_{2}\right) K_{1}+x^{-1} \widetilde{E} K_{1} \\
& +x^{-k}\left(d_{Z} \widetilde{Q}_{1}+\delta_{Z} \widetilde{Q}_{2}\right) K^{\prime}+O\left(\rho_{\mathrm{ff}_{1}}^{-1-b-k f+2 k}\right) .
\end{aligned}
$$


Furthermore, by $(3.33)$ we have that $\Pi_{\mathcal{H}} t\left(\partial_{t}+\Delta\right) \Pi_{\mathcal{H}} K_{1}$ is order $-1-b-k f+1$ since its leading order term solves the model problem.

We assert the existence of a polyhomogeneous distribution $A$ of order $-1-b-k f+$ $k$ such that $t\left(\partial_{t}+\Delta\right)\left(K_{1}-A\right)$ itself vanishes to order $-1-b-k f+1$ at $\mathrm{ff}_{1}$. Indeed, since the leading order term in $t\left(\partial_{t}+\Delta\right)$ is $t x^{-2 k} \Delta^{Z, y}$, and since by 2.33 we can solve $\Delta^{Z, y} B=\left(d_{Z} \widetilde{Q}_{1}+\delta_{Z} \widetilde{Q}_{2}\right) K^{\prime}+\delta_{Z} Q_{1} K_{1}+O\left(\rho_{\mathrm{ff}_{1}}^{-1-b-k f+k}\right)$ where $B$ is polyhomogeneous with asymptotic expansion determined by the expansion of the right hand side, in particular $B=O\left(\rho_{\mathrm{ff}_{1}}^{-1-b-k f}\right)$. We take $A=x^{k} B$ and thus obtain, with $\widetilde{P}$ as in 2.15,

$$
\begin{aligned}
& t\left(\partial_{t}+\Delta\right)\left(K_{1}-x^{k} B\right)= t\left(\partial_{t}+\widetilde{\Delta}_{0}\right)\left(K_{1}-x^{k} B\right)+t x^{-1} \widetilde{E}\left(K_{1}-x^{k} B\right) \\
&-t x^{-k} \widetilde{P} x^{k} B+t O\left(\rho_{\mathrm{ff}_{1}}^{-1-b-k f+2 k}\right) \\
&= t\left(\partial_{t}+\widetilde{\Delta}_{0}\right)\left(K_{1}-x^{k} B\right)+t O\left(\rho_{\mathrm{ff}_{1}}^{-1-b-k f}\right) \\
& \quad+t x^{-1} O\left(\rho_{\mathrm{ff}_{1}}^{-1-b-k f}\right)+t O\left(\rho_{\mathrm{ff}_{1}}^{-1-b-k f}\right) \\
&=O\left(\rho_{\mathrm{ff}_{1}}^{-1-b-k f+1}\right) .
\end{aligned}
$$

Since the expansion of $B$ at $\mathrm{ff}$ has the same order as $K_{1}$, the distribution

$$
K_{2}=K_{1}-x^{k} B
$$

has all of the desired properties of $K$ in the statement of the proposition except that $\left(\partial_{t}+\right.$ $\Delta) K_{2}$ is not rapidly decreasing at lf. Note that, since $\rho_{\mathrm{ff}_{1}}^{1+b+k f} K_{1}=O\left(s^{\bar{\nu}(\mathbf{N})}\right)$ where $\bar{\nu}$ is the (fiber degree dependent) order of $\kappa$ computed in (3.48), by well-posedness $B$ also satisfies $B=O\left(\rho_{\text {lf }}^{\bar{\nu}}\right)$.

To deal with the expansion at lf we argue along similar lines, but there we iterate the argument to get a parametrix $K$ with $\left(\partial_{t}+\Delta\right) K$ vanishes to infinite order at lf. (We work in the interior of If though the arguments at the intersection of If and $\mathrm{ff}_{1}$ are the same in the projective coordinates $s^{\prime}=x / \widetilde{x}, \eta^{\prime}=(y-\widetilde{y}) / \widetilde{x}, \tau^{\prime}=t / \widetilde{x}^{2}$ together with $z, \widetilde{x}, \widetilde{y}, \widetilde{z}$.) Recall that $K_{1} \equiv \kappa$ near lf and thus $K_{2}=\kappa-x^{k} B$ near $\mathrm{ff}_{1}$. Again with $\widetilde{P}$ as in $(2.15)$, we have

$$
\begin{aligned}
\left(\partial_{t}+\Delta\right) K_{2} & =x^{-k} \widetilde{P} \kappa+x^{k-2} \widetilde{E} \kappa-x^{-k} \Delta^{Z, y} B-x^{-k} \widetilde{P} x^{k} B+O\left(x^{\bar{\nu}+k}\right) \\
& =x^{-k}\left(d_{Z} Q_{1}+\delta_{Z} Q_{2}\right) \kappa-x^{-k} \Delta^{Z, y} B+O\left(x^{\bar{\nu}+k-2}\right),
\end{aligned}
$$

where $\bar{\nu}$ is the leading order power of $\kappa$ computed in (3.48). As in the argument at $\mathrm{ff}_{1}$, since the RHS of (3.61) manifestly gives that $\Pi_{\mathcal{H}}\left(\left(\partial_{t}+\Delta\right) K_{2}\right)=O\left(x^{\bar{\nu}+k-2}\right)$, by Section 2.3 there is distribution $A_{0}$ such that $x^{\bar{\nu}} \Delta^{Z, y} A_{0}=\left(d_{Z} Q_{1}+\delta_{Z} Q_{2}\right) \kappa-\Delta^{Z, y} B+O\left(x^{\bar{\nu}+k}\right)$. Here the $x^{\bar{\nu}}$ in front makes it so that $A_{0}$ is $O(1)$. Thus

$$
\left(\partial_{t}+\Delta\right)\left(K_{2}-x^{\bar{\nu}+k} A_{0}\right)=O\left(x^{\bar{\nu}+k-2}\right)-x^{-k} \widetilde{P} x^{\bar{\nu}+k} A_{0}=O\left(x^{\bar{\nu}}\right) .
$$

We will now solve away iteratively to decrease the order of the error. For this we assume for the moment that we are given, for some $q>\bar{\nu}+\epsilon$, any distribution $A_{1}=x^{q} \widetilde{A}_{1}+O\left(x^{q+\epsilon}\right)$ with $\widetilde{A}_{1}$ smooth and non-vanishing up to the boundary as in ice-form. First, we find a distribution $B_{1}$ so that $x^{q} A_{2}:=\left(\partial_{t}+\Delta\right)\left(x^{q+2 k} B_{1}\right)-A_{1}$ is fiber harmonic. We can do this by solving $\left(I-\Pi_{\mathcal{H}}\right) A_{1}=\Delta^{Z, y} B_{1}+O\left(x^{k}\right)$ as in Section 2.3 , where $\Pi_{\mathcal{H}}$ is the projection onto the fiber harmonic forms, since then $\left(\partial_{t}+\Delta\right) x^{q+2 k} B_{1}=x^{q} \Delta^{Z, y} B_{1}+O\left(x^{q+k}\right)$. We then construct a term $C_{1}$ with $\left(\partial_{t}+\Delta\right) x^{q+2} C_{1} \approx A_{2}$, as follows. Decomposing $A_{2}=\left(A_{2}^{1}, A_{2}^{2}\right)$ as in 2.6) in the left varibles, and noting that if $C_{1}=\left(\left(-(q+2)^{2}-(\alpha-1)(q+2)+\right.\right.$ 
$\left.\beta)^{1} A_{2}^{-1},\left(-(q+2)^{2}-(\alpha-1)(q+2)+\gamma\right)^{-1} A_{2}^{2}\right)$, then

$$
\left(\begin{array}{cc}
P_{\alpha, \beta} & 0 \\
0 & P_{\alpha, \gamma}
\end{array}\right) x^{q+2} C_{1}=x^{q} A_{2}
$$

(The numbers we divided by above are non-zero, since the indicial roots of $P_{\alpha, \beta}$ and $P_{\alpha, \gamma}$ are bounded above by $\bar{\nu}-\epsilon$, as explained below (3.48).) For this $C_{1}$ we have

$$
\begin{aligned}
x^{q} A_{2}-\left(\partial_{t}+\Delta\right) x^{q+2} C_{1} & =x^{q} A_{2}-\widetilde{\Delta}_{0} x^{q+2} C_{1}+x^{-k} \widetilde{P}^{\prime} x^{q+2} C_{1}+O\left(x^{q+2}\right) \\
& =O\left(x^{q+\delta}\right)+x^{-k} \widetilde{P}^{\prime} x^{q+2} C_{1}+O\left(x^{q+2+k-2}\right),
\end{aligned}
$$

where $q+\delta$ can be taken to be the order of the subsequent term in the expansion of $A_{2}$ where $\widetilde{\Delta}_{0}$ is in $(3.32)$ and $\widetilde{P}$ is as in $(2.15)$, and thus by Section 2.3 we see that the left hand side lies in the image of $\Delta^{Z, y}$ to order $x^{k}$. We can thus find a distribution $D_{1}$ such that

$$
\begin{aligned}
x^{q} A_{2}-\left(\partial_{t}+\Delta\right)\left(x^{q+2} C_{1}-x^{q+2+k} D_{1}\right) & =O\left(x^{q+1}\right)-x^{q+2-k} \Delta^{Z, y} D_{1}+x^{k} \widetilde{P}^{\prime} x^{q+2} C_{1} \\
& =O\left(x^{q+1}\right),
\end{aligned}
$$

which gives

$$
\left(\partial_{t}+\Delta\right)\left(x^{q}\left(x^{2 k} B_{1}-x^{2} C_{1}+x^{2+k} D_{1}\right)\right)=x^{q} A_{1}+O\left(x^{q+\delta}\right) .
$$

It is straightforward to check that the added terms do not increase the order of blowup at $\mathrm{ff}_{1}$. Thus we can kill off the leading order term of $x^{q} A$, and in fact can kill off all terms iteratively by this process. (If there are log terms present the arguement is analogous and left to the reader.)

From the previous two paragraphs, it follows that we can find a distribution $K^{\prime}$ such that $K:=K_{2}-K^{\prime}$ satisfies the requirements of the lemma, specifically such that $t\left(\partial_{t}+\Delta\right) K$, in addition to having the same leading order asymptotics at tf and $\mathrm{ff}$ and $\mathrm{ff}_{1}$ that $t\left(\partial_{t}+\Delta\right) K_{2}$ has, also vanishes to infinite order at lf. Indeed, since we can solve away terms to obtain errors of succesively decreasing order, taking the Borel sum [29] of these distributions gives $K^{\prime}$.

Proof of Lemma 14. We seek a distribution $K^{\prime}$ with the stated asymptotic properties; namely (3.54)-(3.56) and (3.24) for the indicated $b_{j}$. Such a $K^{\prime}$ will exist by Lemma 21 in Appendix A provided the hypotheses are satisfied, meaning that the following matching conditions hold. We must find a set $\left\{\rho_{\bullet}\right\}$ of boundary defining functions for the boundary hypersurfaces, $\bullet=$ lf, rf, tf, tb, ff, $\mathrm{ff}_{1}$ of $M_{\text {heat }}^{2}$ such that

$$
\begin{aligned}
\kappa_{\mathrm{ff}} & =\left.\frac{1}{(4 \pi)^{n / 2}} \tau^{-n} \sum_{j \in \mathbb{N}} \tau^{j} \widetilde{b}_{j}\right|_{\mathrm{ff}}, \\
\widetilde{x}^{k n} \kappa_{\mathrm{ff}_{1}} & =\widetilde{x}^{1+b+k f} \kappa_{\mathrm{ff}} \text { on } \mathrm{ff} \cap \mathrm{ff}_{1},
\end{aligned}
$$

and that $\kappa_{\mathrm{ff}}, \kappa_{\mathrm{ff}_{1}}$ and the $b_{j}$ vanish to infinite order at tb. Indeed, in the notation of Lemma 21 we have $\kappa_{1}=\left(\rho_{\mathrm{ff}_{1}} / \widetilde{x}\right)^{1+b+k f} \kappa_{\mathrm{ff}_{1}}$ and $\kappa_{2}=\left(\rho_{\mathrm{ff}} / \widetilde{x}\right)^{k n} \kappa_{\mathrm{ff}}$, and the matching conditions in terms of $\kappa_{1}$ and $\kappa_{2}$ in Lemma 21 are exactly (3.63). We use boundary defining functions $\rho_{\mathrm{ff}}=\bar{\rho}, \rho_{\mathrm{ff}_{1}}=\rho / \bar{\rho}$ for the faces ff and $\mathrm{ff}_{1}$ defined in $(3.7)$ and $(3.3)$. We define boundary defining functions $\rho_{\mathrm{lf}}, \rho_{\mathrm{rf}}$ for the faces lf and $\mathrm{rf}$ by the equations

$$
\rho_{\mathrm{lf}} \rho_{\mathrm{ff}} \rho_{\mathrm{ff}}=\widetilde{x}, \quad \rho_{\mathrm{rf}} \rho_{\mathrm{ff}} \rho_{\mathrm{ff}_{1}}=x .
$$

Finally, we use $\tau$ in (3.13) as $\rho_{\mathrm{tf}}$; though it is not valid at tb $\cap \mathrm{tf}$, all the distributions in question will vanish to infinite order there and there will be no conditions to check.

The first matching condition in 3.63 follows easily since the coefficients of the expansion of $\kappa_{\mathrm{ff}_{1}}$ are determined by the same differential equation which determines the $b_{j}$, and the 
coefficients in both expansions are uniquely determined by their being equal to polynomials times Gaussians on the fibers of $\mathrm{tf} \cap \mathrm{ff}$.

Finally we check that the second condition in (3.63) holds. First we consider $\kappa_{\mathrm{ff}_{1}}=$ $\kappa_{\mathrm{ff}_{1}, \widetilde{y}}\left(s, 1, \eta, t^{\prime}\right)$ above the point $\widetilde{y} \in Y$ (i.e. restricted to $\mathrm{ff}_{1 \widetilde{y}}$ ). In the polar coordinates in (3.6) and using the boundary defining functions above (3.64), we have

$$
s=\frac{\bar{\psi}_{x} \rho_{\mathrm{ff}}^{k-1}}{\rho_{\mathrm{lf}}}+1, \quad t^{\prime}=\frac{\bar{\phi}_{t} \rho_{\mathrm{ff}}^{2(k-1)}}{\rho_{\mathrm{lf}}^{2}}, \quad \eta=\frac{\bar{\psi}_{y} \rho_{\mathrm{ff}}^{k-1}}{\rho_{\mathrm{lf}}} .
$$

Using [1, Eqn. 9.7.1], we have that the modified Bessel function satisfies

$$
I_{\nu}(z)=\left(e^{-z} / \sqrt{2 \pi z}\right)(1+O(1 / z)),
$$

and thus

$$
\rho_{\mathrm{ff}}^{k n}\left(\rho_{\mathrm{lf}} \rho_{\mathrm{ff}}\right)^{-1-b-k f} \kappa_{\mathrm{ff}_{1}, \widetilde{y}}=\frac{\rho_{\mathrm{lf}}^{-k f}}{\left(4 \pi \bar{\phi}_{t}\right)^{(b+1) / 2}} e^{-\left(\left.\left|\bar{\psi}_{x}^{2}+\right| \bar{\psi}_{y}\right|_{\widetilde{y}} ^{2}\right) / 4 \bar{\phi}_{t}}\left(1+O\left(\rho_{\mathrm{ff}}\right)\right) .
$$

On the other hand, above each base point $\widetilde{y} \in Y, \kappa_{\mathrm{ff}}, \widetilde{y}\left(\sigma, \eta^{\prime}, z, z^{\prime}, \widetilde{T}\right)$ can be written using separation of variables with respect to the spectrum of $\Delta^{Z, y}$. Indeed, since $H_{Z, y}$ has discrete spectrum, it is standard that $H_{Z, y}(z, \widetilde{z}, t)=\Pi_{0}+E$ where $\Pi_{0}$ is projection onto the kernel of $\Delta^{Z, y}$ and $|E|<e^{-\lambda_{0} t}$ as $t \rightarrow \infty, \lambda_{0}$ being the smallest non-zero eigenvalue of $\Delta_{Z, y}$. Thus

$$
\kappa_{\mathrm{ff}, y}=(2 \pi \widetilde{T})^{-(b+1) / 2} e^{-\left(\sigma^{2}+\left|\eta^{\prime}\right|_{h_{1}}^{2}\right) / 2} \Pi_{0}+E^{\prime},
$$

where $E^{\prime}$ is exponentially decaying. Now we have

$$
\widetilde{T}=\bar{\phi}_{t} \rho_{\mathrm{ff}_{1}}^{-2(k-1)} \rho_{\mathrm{lf}}^{-2 k}, \quad \eta^{\prime}=\bar{\phi}_{t} \rho_{\mathrm{ff}_{1}}^{-(k-1)} \rho_{\mathrm{lf}}^{-k}, \quad \sigma=\bar{\psi}_{x} \rho_{\mathrm{ff}_{1}}^{-(k-1)} \rho_{\mathrm{lf}}^{-k},
$$

and thus

$$
\rho_{\mathrm{ff}_{1}}^{1+b+k f}\left(\rho_{\mathrm{lf}} \rho_{\mathrm{ff}}\right)^{-k n} \kappa_{\mathrm{ff}}=\frac{\rho_{\mathrm{lf}}^{-k f}}{\left(4 \pi \bar{\phi}_{t}\right)^{(b+1) / 2}} e^{-\left(\left|\bar{\psi}_{x}^{2}+\right| \bar{\psi}_{y} \mid \frac{2}{\bar{y}}\right) / 4 \bar{\phi}_{t}},
$$

so the matching condition at $\mathrm{ff} \cap \mathrm{ff}_{1}$ holds.

For the behavior of $K_{1}$ at lf, since $\kappa_{\mathrm{ff}_{1}}$ is given by $\widetilde{x}^{-1-b-k f} \kappa$ at the boundary and we can take $K_{1}$ to be equal to $\kappa$ at lf and at rf.

We can now conclude the proof of Theorem 12 modulo arguments in Appendix B.

Proof of Theorem 12. Having established the existence of a parametrix $K$ as in Proposition 13. we will now prove Theorem 12. We do so by inverting the error $Q=t\left(\partial_{t}+\Delta\right) K$ from Proposition 13 via a Neumann series. To be precise, it will be convenient to think of distributional kernels $A\left(p, p^{\prime}, t\right)$ on $M \times M \times \mathbb{R}^{+}$acting on $C_{c}^{\infty}\left(M^{\circ} \times(0, \infty)\right)$ by operating as convolution kernels in the time variable, so for $\phi \in C_{c}^{\infty}\left(M^{\circ} \times(0, \infty)\right)$ by

$$
(A \star \phi)(p, t):=\int_{M} \int_{0}^{t} A\left(p, p^{\prime}, t-s\right) \phi\left(p^{\prime}, s\right) d s \mathrm{dVol}_{p^{\prime}} .
$$

Then

$$
\left(\partial_{t}+\Delta\right) \widetilde{K}=I+t^{-1} Q
$$

and the right hand side can be inverted via a Neumann series, i.e. $\left(\operatorname{Id}+t^{-1} Q\right)\left(I+Q^{\prime}\right)=\mathrm{Id}$ where $Q^{\prime}=\sum_{j=1}^{\infty}(-1)^{j}\left(t^{-1} Q\right)^{j}$ and $\left(t^{-1} Q\right)^{j}=t^{-1} Q \star \cdots \star t^{-1} Q, j$-times. In Proposition 28 below, we show that the summands $\left(t^{-1} Q\right)^{j}$ vanishes at successively faster orders at $\mathrm{ff}$ and $\mathrm{ff}_{1}$. Moreover, as discussed in 27, 5, this series is convergent in $C^{\infty}$, and the infinite order of vanishing of $t^{-1} Q$ at If is preserved in the sum, i.e. $Q^{\prime}$ vanishes also to infinite 
order there. In fact, one sees as in [27] that $\widetilde{K}\left(I+Q^{\prime}\right)$ is polyhomogeneous with the index set $\mathcal{E}$ statisfying the properties of Theorem 12 .

\section{Spectral and Hodge theoretic properties of the Hodge-Laplacian}

In this section we deduce the main theorems from the introduction. We begin with a detailed analysis of the polyhomogeneous forms in the maximal domain.

4.1. Polyhomogeneous forms in $\mathcal{D}_{\max }$ and $\mathcal{D}_{\min }$. Recall the definition of $\mathcal{D}_{\max }$ and $\mathcal{D}_{\text {min }}$ from the introduction, and the space $\mathcal{A}_{\mathrm{phg}}\left({ }^{\text {ice }} \Lambda^{*}\right.$ ) of polyhomogeneous ice-forms (below denoted simply by $\mathcal{A}_{\mathrm{phg}}$ ) discussed in Section A. We determine conditions which assure that a given polyhomogeneous differential form $\gamma \in \mathcal{A}_{\text {phg }}$ is contained in the maximal domain $\mathcal{D}_{\max }$ of $\Delta^{g}$. This will be used to show, with an additional assumption on the index set of a phg form, that

$$
\gamma \in \mathcal{D}_{\max } \cap \mathcal{A}_{\mathrm{phg}} \Longrightarrow \gamma \in \mathcal{D}_{\min } \cap \mathcal{A}_{\mathrm{phg}} .
$$

Let $\gamma \in \mathcal{A}_{\text {phg }}$ be contained in the maximal domain, i.e. we assume that $\gamma \in L^{2}$ and $\Delta^{g} \gamma \in L^{2}$. Let $\gamma=x^{s} \tilde{\gamma}$ where $\tilde{\gamma}=\tilde{\gamma}_{0}(y, z)+\mathcal{O}\left(x^{\epsilon}\right)$. Here notation such as $\mathcal{O}\left(x^{\epsilon}\right)$ indicates that the differential form $\gamma$ is locally a combination of basis forms

$$
d y_{I} \wedge x^{k \mathbf{N}} d z_{A} \quad \text { and } \quad d x \wedge x^{k \mathbf{N}} d y_{I} \wedge x^{k} \mathbf{N} d z_{A},
$$

where $I$ and $A$ are multi-indices on the base and fiber, respectively. with coefficient functions which are bounded by $c x^{\epsilon}$ pointwise in norm when $x \searrow 0$, and $\tilde{\gamma}_{0}$ is a form on $M$ whose coefficient functions are independent of $x$. Let us determine the possible range of values $s$. From 2.9 it follows that in a neighborhood of the boundary, the volume form of the cuspedge metric $g$ is

$$
\mathrm{dVol}_{g}=x^{k f} \rho d x \wedge d y \wedge d z
$$

where $\rho=a(y, z)+\mathcal{O}\left(x^{k}\right)$ and $a$ is a non-vanishing positive function. It follows that

$$
x^{s} \tilde{\gamma} \in L^{2}(M, g) \Longleftrightarrow s>-\frac{1}{2}(k f+1) .
$$

We begin by analyzing the indicial roots of $\Delta^{g}$, specifically we find the order of vanishing of approximately fiber harmonic homogeneous forms in the kernel of $\Delta^{g}$. By Proposition 7 the leading order part of $\Delta^{g}$ restricted to approximately fiber harmonic forms (see Section 2.3 is

$$
\Pi_{\mathcal{H}} \Delta_{0}^{g} \Pi_{\mathcal{H}} \sim:=\left(\begin{array}{cc}
P_{\alpha(\mathbf{N}), \beta(\mathbf{N})} & 0 \\
0 & P_{\alpha(\mathbf{N}), \gamma(\mathbf{N})}
\end{array}\right),
$$

with $P_{\alpha(\mathbf{N}), \beta(\mathbf{N})}, P_{\alpha(\mathbf{N}), \gamma(\mathbf{N})}$ the operators, depending on fiber degree, defined in 3.34 -3.35 We note that

$$
P_{\alpha(f-\mathbf{N}), \beta(f-\mathbf{N})}=P_{\alpha(\mathbf{N}), \gamma(\mathbf{N})} \quad(\mathbf{N}=0, \ldots, f) .
$$

By (3.39), the values $s$ for which $P_{\alpha(\mathbf{N}), \beta(\mathbf{N})} x^{s}=0$ and for which elements of size $x^{s}$ are in addition contained in $L^{2}(M, g)$ is

$$
s= \begin{cases}-k \mathbf{N} & \text { if } \mathbf{N}<\frac{1}{2}\left(f+\frac{1}{k}\right) \\ 1-k(f-\mathbf{N}) & \text { if } \mathbf{N}>\frac{1}{2}\left(f-\frac{3}{2 k}\right) .\end{cases}
$$

Proposition 15. Suppose the differential form $\gamma=\left(\gamma^{1}, \gamma^{2}\right)=\left(x^{s_{1}} \tilde{\gamma}^{1}, x^{s_{2}} \tilde{\gamma}^{2}\right) \in \mathcal{A}_{\text {phg }}$ and that $\tilde{\gamma}^{j}=\tilde{\gamma}_{0}^{j}(y, z)+\mathcal{O}\left(x^{\epsilon}\right)$ is contained in the maximal domain $\mathcal{D}_{\max }$. (Thus the leading order term is assumed not to have a logarithm, as is a priori allowed for phg-distributions.) 
Then each $s_{j}$ is an indicial root of $P_{\alpha(\mathbf{N}), \beta(\mathbf{N})}$ for some $0 \leq \mathbf{N}_{j} \leq f$ or $s_{j}>\frac{1}{2}(-k f+3)$. In either case, $s_{j} \geq \frac{1}{2}(-k f+3)$.

Proof. Recall form Proposition 7 that

$$
\Delta^{g}=\Delta_{0}+x^{-k} \widetilde{P}+x^{-1} \widetilde{E},
$$

where

$$
\Delta_{0}=\left(\begin{array}{cc}
P_{\alpha(\mathbf{N}), \beta(\mathbf{N})} & 0 \\
0 & P_{\alpha(\mathbf{N}), \gamma(\mathbf{N})}
\end{array}\right)+\left(\begin{array}{cc}
\frac{1}{x^{2 k}} \Delta^{Z, y}+\Delta_{Y} & -2 k x^{-k-1} d^{Z} \\
-2 k x^{-k-1} \delta^{Z} & \frac{1}{x^{2 k}} \Delta^{Z, y}+\Delta_{Y}
\end{array}\right) .
$$

In view of the symmetry 4.3 it suffices to consider the image of the the component $\gamma^{1}=$ $x^{s_{1}} \tilde{\gamma}^{1}$ under $\Delta^{g}$. The discussion naturally falls into several cases.

(1) The form $\tilde{\gamma}_{0}^{1}$ is not approximately fiber harmonic in the sense of $\$ 2.3$. Then the lowest nonvanishing term in 4.5 is $x^{-2 k+s_{1}} \Delta^{Z} \tilde{\gamma}_{0}^{1}$, which is contained in $L^{2}$ if and only if

$$
s_{1}>\frac{1}{2}(3 k f-1)
$$

(2) The form $\tilde{\gamma}_{0}^{1}$ is approximately fiber harmonic harmonic. We then consider the following subcases.

(2.a) $s_{1}$ is an indicial root of $P_{\alpha\left(\mathbf{N}_{1}\right), \beta\left(\mathbf{N}_{1}\right)}$ and hence equals the number in 4.4.

(2.b) $s_{1}$ is not an indicial root of $P_{\alpha\left(\mathbf{N}_{1}\right), \beta\left(\mathbf{N}_{1}\right)}$, i.e. $P_{\alpha\left(\mathbf{N}_{1}\right), \beta\left(\mathbf{N}_{1}\right)}\left(x^{s_{1}} \tilde{\gamma}^{1}\right) \neq 0$. We claim that at least one of the following two statements holds true:

* The polyhomogeneous expansion of $\tilde{\gamma}^{1}$ contains a term $\tilde{\gamma}_{\ell}^{1}$ of order $\mathcal{O}\left(x^{\delta}\right)$ where $\delta-2 k<s_{1}-2$ and $\tilde{\gamma}_{\ell}^{1}$ is not approximately fibre harmonic.

* The lowest nonvanishing term in the first component of $\Delta^{g} \gamma$ is of order $x^{s_{1}-2}$.

If this claim holds true we conclude that the lowest nonvanishing term in the first component of $\Delta^{g} \gamma$ is of order at most $x^{s_{1}-2}$. To prove the claim, assume that the first statement is false. Then the second one must hold true as is clear from the form of the Laplacian $\Delta_{0}$ in 4.5 . To be specific, collecting the terms of order $x^{s_{1}-2}$ in the first component of $\Delta_{0} \gamma$ we obtain

$$
\begin{aligned}
P_{\alpha\left(\mathbf{N}_{1}\right), \beta\left(\mathbf{N}_{1}\right)}\left(x^{s_{1}} \tilde{\gamma}^{1}\right)+x^{-2 k} \Delta^{Z} \tau^{1}+x^{-k-1} d^{Z} \tau^{2}+x^{-k} d_{Z} Q_{2} \tau^{3}+x^{-k} Q_{4} d_{Z} \tau^{4} \\
+x^{-k} \delta_{Z} Q_{1} \tau^{5}+x^{-k} Q_{3} \delta_{Z} \tau^{6}
\end{aligned}
$$

for suitable differential forms $\tau^{1}, \ldots, \tau^{6}$ of orders

$$
\tau^{1}=O\left(x^{s_{1}+2 k-2}\right), \quad \tau^{2}=O\left(x^{s_{1}+k-1}\right), \text { and } \tau^{j}=O\left(x^{s_{1}+k-2}\right) \quad(j=3,4,5,6) .
$$

By Hodge theory, the terms $d_{Z} \tau^{4}$ and $\delta_{Z} \tau^{6}$ both vanish approximately in the sense of $\$ 2.3$ since otherwise a nonvanishing term $x^{-2 k} \Delta^{Z} \tau^{4}$, respectively $x^{-2 k} \Delta^{Z} \tau^{5}$ would occur. These are both of order strictly less than $s_{1}-2$, contradicting our initial assumption. Considering the remaining five terms in (4.6) it follows from Hodge theory and the assumption that $\tilde{\gamma}_{0}^{1}$ is approximately fibre harmonic that the sum

$$
x^{-2 k} \Delta^{Z} \tau^{1}+x^{-k-1} d^{Z} \tau^{2}+x^{-k} d_{Z} Q_{2} \tau^{3}+x^{-k} \delta_{Z} Q_{1} \tau^{5}
$$

is approximately orthogonal to $P_{\alpha\left(\mathbf{N}_{1}\right), \beta\left(\mathbf{N}_{1}\right)}\left(x^{s_{1}} \tilde{\gamma}^{1}\right)$ in the sense of 2.3 . Hence we conclude that the nonzero term $P_{\alpha\left(\mathbf{N}_{1}\right), \beta\left(\mathbf{N}_{1}\right)}\left(x^{s_{1}} \tilde{\gamma}^{1}\right)$ cannot cancel with the sum (4.7). It follows that the second statement is true, whence the claim. 
The asserted statement follows by inspection of each of the above cases. In case (1) it follows from

$$
s_{1}>\frac{1}{2}(3 k f-1)>\frac{1}{2}(-k f+3),
$$

using that $k \geq 3$. In case (2.b) the lowest nonvanishing term in $\Delta^{g} \gamma$ is of order at most $s_{1}-2$. Since $\gamma \in \mathcal{D}_{\max }$ it follows from 4.2 that

$$
s_{1}-2>-\frac{1}{2}(k f+1) \Longleftrightarrow s_{1}>-\frac{1}{2}(k f+3) .
$$

In case (2.a), the form $\tilde{\gamma}_{0}^{1}$ is approximately fibre harmonic and therefore by the Witt condition $\mathbf{N} \neq \frac{f}{2}$. The exponent $s_{1}$ is given by 4.4 from which it follows that if $f$ is even that

$$
s_{1} \geq-k\left(\frac{f}{2}-1\right)
$$

(here we use the Witt condition) and if $f$ is odd that

$$
s_{1} \geq-k\left(\frac{f}{2}-\frac{1}{2}\right) \geq-\frac{k f}{2}+\frac{3}{2}
$$

where the last inequality follows from the assumption $k \geq 3$.

Lemma 16. Assume $k \geq 3$. Then $\mathcal{D}_{\min } \cap \mathcal{A}_{\mathrm{phg}}=\mathcal{D}_{\max } \cap \mathcal{A}_{\mathrm{phg}}$.

Proof. It suffices to prove the inclusion $\mathcal{D}_{\max } \cap \mathcal{A}_{\text {phg }} \subseteq \mathcal{D}_{\text {min }} \cap \mathcal{A}_{\text {phg }}$. For $\varepsilon>0$ we define the logarithmic cutoff function $\chi_{\varepsilon}:[0, \infty) \rightarrow[0,1]$ by

$$
\chi_{\varepsilon}(x):= \begin{cases}0, & x \leq \varepsilon^{2}, \\ -\frac{\log \left(x / \varepsilon^{2}\right)}{\log (\varepsilon)}, & \varepsilon^{2}<x<\varepsilon, \\ 1, & x \geq \varepsilon .\end{cases}
$$

For $\varepsilon^{2}<x<\varepsilon$ it satisfies

$$
\chi_{\varepsilon}^{\prime}(x)=-\frac{1}{\log (\varepsilon) x} \quad \text { and } \quad \chi_{\varepsilon}^{\prime \prime}(x)=\frac{1}{\log (\varepsilon) x^{2}} .
$$

Let $\gamma \in \mathcal{D}_{\max } \cap \mathcal{A}_{\text {phg }}$ and set $\gamma_{\varepsilon}=\chi_{\epsilon} \gamma$. Then

$$
\begin{aligned}
\Delta^{g} \gamma_{\varepsilon}=\chi_{\varepsilon} \Delta^{g} \gamma-\left(\partial_{x}^{2} \chi_{\varepsilon}\right) \gamma-\left(\partial_{x} \chi_{\varepsilon}\right)\left(\partial_{x} \gamma\right)- & \frac{k f}{x}\left(\partial_{x} \chi_{\varepsilon}\right) \gamma \\
& +A^{j}\left(\partial_{x} \chi_{\varepsilon}\right)\left(\partial_{z_{j}} \gamma\right)+B^{i}\left(\partial_{x} \chi_{\varepsilon}\right)\left(\partial_{y_{i}} \gamma\right),
\end{aligned}
$$

where $A^{j}=\mathcal{O}\left(x^{k}\right)$ and $B^{i}=\mathcal{O}\left(x^{2 k}\right)$ are bounded functions with that order of decay in $x$. We show that

$$
\left\|\Delta^{g} \gamma_{\varepsilon}-\Delta^{g} \gamma\right\|_{L^{2}(M, g)} \rightarrow 0 \quad \text { as } \quad \varepsilon \rightarrow 0
$$

hence establishing that $\gamma \in \mathcal{D}_{\text {min }}$. It is clear that

$$
\left\|\chi_{\varepsilon} \Delta^{g} \gamma-\Delta^{g} \gamma\right\|_{L^{2}(M, g)} \rightarrow 0 \quad \text { as } \quad \varepsilon \rightarrow 0,
$$

and thus it suffices to consider the next three terms in 4.9 ) and to show that

$$
\frac{1}{\log ^{2}(\varepsilon)} \int_{\varepsilon^{2}}^{\varepsilon} \frac{1}{x^{4}}|\gamma|^{2} x^{k f} d x+\frac{1}{\log ^{2}(\varepsilon)} \int_{\varepsilon^{2}}^{\varepsilon} \frac{1}{x^{2}}\left|\partial_{x} \gamma\right|^{2} x^{k f} d x+\frac{k^{2} f^{2}}{\log ^{2}(\varepsilon)} \int_{\varepsilon^{2}}^{\varepsilon} \frac{1}{x^{4}}|\gamma|^{2} x^{k f} d x
$$


converges to 0 as $\varepsilon \rightarrow 0$. Let $\gamma=x^{s} \tilde{\gamma}$ for some $\tilde{\gamma}=\mathcal{O}(1)$. A short calculation shows that each integrand in (4.11) is of order $x^{-1+\delta}$ for some $\delta>0$ and hence converges to 0 as $\varepsilon \rightarrow 0$ if

$$
s>-\frac{k f}{2}+\frac{3}{2}
$$

In the borderline case $s=-\frac{k f}{2}+\frac{3}{2}$ we still get convergence since then the first integral in (4.11) becomes

$$
\frac{1}{\log ^{2}(\varepsilon)} \int_{\varepsilon^{2}}^{\varepsilon} \frac{1}{x} d x=\frac{1}{\log ^{2}(\varepsilon)}\left(\log (\varepsilon)-\log \left(\varepsilon^{2}\right)\right)=-\frac{1}{\log (\varepsilon)} \rightarrow 0 \quad \text { as } \varepsilon \rightarrow 0,
$$

and analogously for the second and third integral. Hence

$$
s \geq-\frac{k f}{2}+\frac{3}{2} \Longrightarrow \gamma \in \mathcal{D}_{\text {min }}
$$

for any $\gamma=x^{s} \tilde{\gamma} \in \mathcal{D}_{\max } \cap \mathcal{A}_{\text {phg. }}$. On the other hand, Proposition 15 shows that

$$
\gamma=x^{s} \tilde{\gamma} \in \mathcal{D}_{\max } \cap \mathcal{A}_{\mathrm{phg}} \quad \Longrightarrow \quad s \geq-\frac{k f}{2}+\frac{3}{2},
$$

and hence the claim follows.

\subsection{Spectral theory.}

Proof of Theorem 4. The existence of a fundamental solution $H_{t}$ is the content of Theorem 12. so it suffices to show that $H_{t}$ has the properties stated in Theorem 4 . Since $H_{t}$ and $\partial_{t} H_{t}$ are formally self-adjoint (i.e. symmetric), to show that they are self-adjoint it suffices to show that they are compact operators. But indeed they are, as follows from 33 , Thm VI.23-24] together with

$$
H_{t}, \partial_{t} H_{t} \in L^{2}(\text { End; } M \times M),
$$

where, given a smooth section $A$ of End, then $A \in L^{2}($ End; $M \times M)$ if

$$
\int\|A(p, q)\|_{\text {End }}^{2} \mathrm{dVol}_{M}(p) \mathrm{dVol}_{M}(q)<\infty .
$$

For $t>0, H_{t}$ is given by an $L^{2}$ integral kernel, so is a compact operator; indeed, by (3.57), the index set $\mathcal{F}$ of $H_{t} \in \mathcal{A}_{\text {phg }}(M \times M)$ restricted to $t>0$ constant is $\mathcal{F}$ (lf) $=\mathcal{E}$ (lf) and $\mathcal{F}(\mathrm{rf})=\mathcal{E}(\mathrm{rf})$, for $\mathcal{E}$ the index family of $H$. From (3.58), these satisfy the lower bound

$$
\inf \mathcal{F}(\text { lf }), \text { inf } \mathcal{F}(\text { rf }) \geq-\frac{k f}{2}+1
$$

(meaning $H_{t}$ is a bounded endomorphism) and

$$
\mathrm{dVol}_{M}(p) \mathrm{dVol}_{M}(q) \simeq x^{k f} \widetilde{x}^{k f} d x d \widetilde{x} d y d \widetilde{y} d z d \widetilde{z},
$$

so the kernel of $H_{t}$ is square integrable. Since the restriction of $\partial_{t} H_{t}$ to a fixed time $t$ has the same index set on $M \times M$ as $H_{t}$, it is also compact.

It remains to establish (4), i.e. that $H_{t}(\alpha) \in \mathcal{D}_{\min }$ for every $\alpha \in L^{2}$. In fact, $H_{t}(\alpha)$ is a polyhomogeneous distribution with index set $\mathcal{E}($ lf). This is straightforward: writing the expansion of $H_{t}$ at $x=0$ up to some order $N$ we have

$$
H_{t}=\sum_{\substack{(s, p) \in \mathcal{E}(\mathrm{lf}) \\|s| \leq N}} x^{s} \log ^{p}(x) a_{s, p}(y, z, \widetilde{w})+E_{N}
$$


where $\widetilde{w}=(\widetilde{x}, \widetilde{y}, \widetilde{z})$, and the coefficients $a_{s, p}$ are polyhomogeneous endomorphisms on the manifold with boundary $\partial M \times M$ and $E_{N}$ is a polyhomogeneous endomorphism on $M \times M$ with $E_{N}=o\left(x^{N}\right)$. Thus

$$
\left.H_{t}(\alpha)=\int_{M}\left(\sum_{\substack{(s, p) \in \mathcal{E}(\mathrm{lf}) \\ \Re s \leq N}} x^{s} \log ^{p}(x) a_{s, p}(z, y, \widetilde{w}) \alpha(\widetilde{w})+E_{N} \alpha(\widetilde{w})\right)\right) \mathrm{dVol}_{g}(\widetilde{w}) .
$$

For example by [25, Proposition 3.20], since the $x^{-N} E_{N}$ are given by a polyhomogeneous integral kernel, they define bounded maps of $L^{2}$, and the conormality estimates (see A.3)(A.4)) follow by differentiating $x^{-N} E_{N}$. The integrals coming from the partial expansion terms are finite and give the expansion coefficients of $H_{t}(\alpha)$. This shows that $H_{t}(\alpha) \in \mathcal{A}_{\mathrm{phg}}$, and moreover that the leading order term has no logarithmic factor. Thus, In view of Lemma 16 it suffices to prove $H_{t}(\alpha) \in \mathcal{D}_{\max }$ in order to conclude that $H_{t}(\alpha) \in \mathcal{D}_{\min }$. But indeed, $\inf \mathcal{E}$ (lf) satisfies the lower bound (4.13), hence it follows that the lowest order term in the polyhomogeneous expansion 4.15 is of order at least $-\frac{k f}{2}+1$ which by 4.2 is sufficient to conclude $H_{t}(\alpha) \in L^{2}$. Because $H_{t}$ is a fundamental solution of the heat equation, it follows that $\Delta^{g} H_{t}(\alpha)=-\partial_{t} H_{t}(\alpha)$ which by the same argument is contained in $L^{2}$ since $\partial_{t} H_{t}$ has the same index set as $H_{t}$ for $t>0$.

It now follows that the fundamental solution $H_{t}$ from Theorem 12 is in fact the heat kernel in the following sense.

Proposition 17. The heat kernel $\exp \left(-t \Delta^{g}\right)$ defined by applying the spectral theorem to the self-adjoint operator $\left(\Delta^{g}, \mathcal{D}\right)$ has Schwartz kernel equal to the fundamental solution $H_{t}$ in Theorem 12, meaning

$$
\left(e^{-t \Delta^{g}} \alpha\right)(w)=\int_{M} H_{t}(w, \widetilde{w}, t) \alpha(\widetilde{w}) \operatorname{dVol}_{g}(\widetilde{w}) .
$$

Using this we may finish the proof of Theorem 1 .

Proof of Theorem 1. As discussed below the statement of Theorem 4, Theorem 4 itself establishes essential self-adjointness of $\Delta^{g}$. It remains to prove that the spectrum is discrete, but this follows immediately from the spectral theorem and the fact that $H_{t}$ is a compact operator (hence has discrete spectrum.)

Moreover, the Weyl asymptotic formula in (1.6) follows from the standard heat kernel argument in [35, §8.3] together with the heat trace asymptotics in Corollary 18

Corollary 18 (Heat trace asymptotics). For each $t>0$, the fundamental solution $H_{t}$ in Theorem 12 is trace class and satisfies that $F(t):=\operatorname{Tr} H_{t}$ is a polyhomogenous conormal distribution on $\mathbb{R}^{+}$satisfying

$$
\begin{aligned}
F(t)=t^{-n / 2} & \operatorname{Vol}(M, g)+\left(\sum_{j=1}^{\infty} a_{j} t^{-n / 2+j}\right)+c_{0} t^{-(b+1) / 2+1 / 2 k} \\
+ & O\left(t^{-(b+1) / 2+1 / 2 k+\epsilon}\right) .
\end{aligned}
$$

The proof of Corollary 18, which uses Theorem 12 and Melrose's pushforward theorem, is deferred to Section A.2 below. 
4.3. Harmonic forms and Hodge theory. We begin our discussion of Hodge theory by pointing out that elements $\gamma \in L^{2}$ satisfying $\Delta^{g} \gamma=0$, admit asymptotic expansions at the boundary of $M$. Indeed, for such forms $\gamma$, by the spectral theorem and the fact that $H_{t}$ is the heat kernel (Corollary 17), we see that

$$
\gamma=H_{t} \gamma=\int_{M} H_{t}(w, \widetilde{w}, t) \gamma(\widetilde{w}) \operatorname{dVol}_{g}(\widetilde{w}) .
$$

By the proof of Theorem 4, specifically 4.15), we have the following.

Lemma 19. Assume that $\gamma \in \operatorname{ker}\left(\Delta^{g}: L^{2} \longrightarrow L^{2}\right)$. Then $\gamma$ is polyhomogeneous conormal and $\gamma=\mathcal{O}(1)$, i.e. is bounded in norm.

Lemma 19 allows us to conclude that the $L^{2}$ kernel of $\Delta^{g}$ is equal to the Hodge cohomology in 1.7$)$.

Lemma 20. Notation as above, $\mathcal{H}_{L^{2}}(M, g)=\operatorname{ker}\left(\Delta^{g}: L^{2} \longrightarrow L^{2}\right)$.

Proof. If $\gamma \in \mathcal{H}_{L^{2}}(M, g)$ then $\gamma$ is in the maximal domains of both $d$ and $\delta$, and so for smooth compactly supported $\beta$,

$$
\left\langle\Delta^{g} \gamma, \beta\right\rangle_{L^{2}}:=\left\langle\gamma, \Delta^{g} \beta\right\rangle_{L^{2}}=\langle\gamma, d \delta \beta\rangle_{L^{2}}+\langle\gamma, \delta d \beta\rangle_{L^{2}}=0+0=0,
$$

so $\gamma \in \operatorname{ker}\left(\Delta^{g}: L^{2} \longrightarrow L^{2}\right)$

On the other hand, if $\gamma \in \operatorname{ker}\left(\Delta^{g}: L^{2} \longrightarrow L^{2}\right)$, then by Lemma 19 we can integrate by parts to obtain

so $\gamma \in \mathcal{H}_{L^{2}}^{*}(M, g)$.

$$
0=\left\langle\Delta^{g} \gamma, \gamma\right\rangle_{L^{2}}=\|d \gamma\|_{L^{2}}^{2}+\|\delta \gamma\|_{L^{2}}^{2}
$$

We can now follow the arguments in [17, 19] to prove Theorem 22 above. Before we begin we recall some facts about intersection cohomology, a cohomology theory that applies to stratified spaces. We do not attempt to make a full explanation of it here, but mention only that there is in fact a family of intersection cohomology groups for our stratified space $X$ defined in (1.8) (obtained by collapsing the boundary of $\partial M$ over the base $Y$ ) depending on a function $\mathfrak{p}: \mathbb{N} \longrightarrow \mathbb{N}$ called the 'perversity,' which is non-decreasing and whose values matter only on the codimensions of the strata of $X$. Here we have only one singular stratum, $Y \subset X$, the image of the boundary $\partial M$ via the projection onto $X$, and its codimension is $f+1$, where $\operatorname{dim} Z=f$. The 'upper middle degree' perversity $\overline{\mathfrak{m}}$ is a special example of a perversity, which satisfies

$$
\overline{\mathfrak{m}}(f+1)=\left\{\begin{array}{cc}
(f-1) / 2 & \text { if } f \text { is odd } \\
f / 2-1 & \text { if } f \text { is even. }
\end{array}\right.
$$

The 'lower middle perversity' $\mathfrak{m}$ differs from $\overline{\mathfrak{m}}$ only when $f$ is even, in which case $\underline{\mathfrak{m}}(f+1)=$ $f / 2$. As we will rely on the spectral sequence arguments from [17, 19] during the proof, we will only need to study the intersection cohomology locally, specifically on a basis of open sets of $X$. Concretely, from [17, for canonical neighborhoods $U=V \times C_{1}(Z)$ as in 2.4. with contractible $V$, we have

$$
I H_{\mathfrak{p}}^{p}(U)=\left\{\begin{array}{cl}
H^{p}(Z) & \text { if } p<f-1-\mathfrak{p}(f+1), \\
\{0\} & \text { if } p \geq f-1-\mathfrak{p}(f+1) .
\end{array}\right.
$$

From the Witt condition 1.4, we see that

$$
I H_{\overline{\mathfrak{m}}}^{p}(U)=I H_{\underline{\mathfrak{m}}}^{p}(U)=\left\{\begin{array}{cc}
H^{p}(Z) & \text { if } p<f / 2, \\
\{0\} & \text { if } p \geq f / 2,
\end{array}\right.
$$


regardless of the parity of $f$.

Proof of Theorem 2. Although Theorem 2 describes a relationship between the Hodge cohomology and the intersection cohomology, to prove it we go through the standard route and use the intermediary of $L^{2}$-cohomology. Thus consider the chain complex

$$
\ldots \longrightarrow L_{d}^{2} \Omega^{p-1}(M, g) \longrightarrow L_{d}^{2} \Omega^{p}(M, g) \longrightarrow L_{d}^{2} \Omega^{p+1}(M, g) \longrightarrow \ldots
$$

where $L_{d}^{2} \Omega^{p}(M, g)$ is the maximal domain of the exterior derivative $d$, specifically

$$
L_{d}^{2} \Omega^{p}(M, g)=\left\{\alpha \in L^{2} \Omega^{p}(M, g): d \alpha \in L^{2} \Omega^{p+1}(M, g)\right\} .
$$

Then the $L^{2}$-cohomology is the quotient

$$
L^{2} H^{p}(M, g)=\frac{\left\{\alpha \in L_{d}^{2} \Omega^{p}(M, g): d \alpha=0\right\}}{\left\{d \eta: \eta \in L_{d}^{2} \Omega^{p-1}(M, g)\right\}} .
$$

As explained in [19, page 6], it suffices to show that

$$
L^{2} H^{p}(M, g) \simeq I H_{\overline{\mathrm{m}}}^{p}(X ; \mathbb{R}),
$$

for then the $L^{2}$-cohomology is finite dimensional, which implies that the range of $d$ (and thus its adjoint $\delta$ ) is closed. From [17, §2.1] it then follows using the Kodaira decomposition theorem that $\mathcal{H}_{L^{2}}^{p}(M, g)$ is isomorphic to $L^{2} H^{p}(M, g)$ and thus by 4.21) Theorem 2 holds.

Thus it suffices to prove (4.21), and for this we also follow the arguments in [19, pp. 5-6], where it is explained that it suffices to show that for canonical neighborhoods $U=V \times C_{1}(Z)$ as in 2.4 with contractible $V$, the local chain complex

$$
\ldots \longrightarrow L_{d}^{2} \Omega^{p-1}(U, g) \longrightarrow L_{d}^{2} \Omega^{p}(U, g) \longrightarrow L_{d}^{2} \Omega^{p+1}(U, g) \longrightarrow \ldots,
$$

satisfies

$$
L^{2} H^{p}(U, g) \simeq I H_{\frac{p}{\mathfrak{m}}}(U)
$$

Here $L^{2} H^{p}(U, g)$ is defined as above with $U$ replacing $M$. The intersection cohomology groups for $\overline{\mathfrak{m}}$ are computed in 4.19, and thus we need only to analyze the groups on the left. To see [4.23 we use the Künneth formula of Zucker, [38, Corollary 2.34], whose assumptions are satisfied here by the fact that the exterior derivative on $Z$ is closed on its maximal domain. Thus, in the notation of [19, Page 5], we have

$$
\left.L^{2} H^{p}(U, g)=\bigoplus_{i=0}^{1} W H^{i}\left((0,1), d x^{2}, k(p-i-f / 2)\right)\right) \otimes H^{p-i}(Z ; \mathbb{R}),
$$

where $W H^{i}\left((0,1), d x^{2}, a\right)$ is the cohomology of the complex

$$
0 \longrightarrow\left(x^{a} L^{2} \Omega^{0}\left((0,1), d x^{2}\right)\right) \stackrel{d}{\longrightarrow} x^{a} L^{2} \Omega^{1}\left((0,1), d x^{2}\right) \longrightarrow 0,
$$

where the space on the left is the maximal domain of $d$ on $x^{a} L^{2} \Omega^{0}\left((0,1), d x^{2}\right)$. Again from [19] (via [17]), $W H^{1}\left((0,1), d x^{2}, a\right)=0$ if $a \neq 1 / 2$ and $W H^{0}\left((0,1), d x^{2}, a\right)=\mathbb{R}$ if $a<1 / 2$ and $\{0\}$ if $a \geq 1 / 2$. When $i=1, k(p-i-f / 2) \neq 1 / 2$ since $k>1$, so the $i=1$ terms do not contribute. When $i=0$, we have $k(p-i-f / 2)=k(p-f / 2)$ which satisfies

$$
k(p-f / 2)<1 / 2 \text { if } p \leq f / 2 \text { and } k(p-f / 2)>1 / 2 \text { if } p>f / 2 .
$$

Using the Witt condition then gives

$$
L^{2} H^{p}(U, g)=\left\{\begin{array}{cc}
H^{p}(Z) & \text { if } p<f / 2 \\
\{0\} & \text { if } p \geq f / 2
\end{array}\right.
$$

matching (4.19) and completing the proof. 
We now discuss the proof of Theorem 3 . As the spaces in the theorem are incomplete cusp edge spaces in a neighborhood of the divisor by [28], our results would apply to these spaces, if not for the fact that moduli spaces such as these have interior orbifold points. This is not a problem, since, as in 20 we may lift to a finite cover with no such points. One can then work on the space $C_{c . \text { orb }}^{\infty}\left(\mathcal{M}_{1,1}\right)$ of functions which near each orbifold point are smooth when lifted to a local finite cover resolving the singularity. Constructing a heat kernel on the lift and averaging over the group action then gives a fundamental solution to the heat kernel downstairs which has all the desired properties. We leave the details of this simple extension to the reader.

\section{Appendix A. Manifolds With CORners}

In this section we recall some of the facts about distributions on manifolds with corners (mwc's) used in this paper. This material is due largely to Melrose, and the reader is referred to his book 31 for more details. See also [18.

The objects considered here, for example the ice-metrics 2.9 , have polyhomogeneous regularity, which we define now. The sheaf of polyhomogeneous conormal (or polyhomogeneous, or simply phg) functions $\mathcal{A}_{\text {phg }}(X)$ is defined as follows. First, an index set $\mathcal{E}$ on a manifold with corners $X$ is an association to each boundary hypersurface $H$ of $X$ a set

$$
\begin{gathered}
\mathcal{E}(H) \subset \mathbb{C} \times \mathbb{N} \text { satisfying that the subset } \\
\{(z, p) \in \mathcal{E}(H): \operatorname{Re} z<c\} \text { is finite for all } c \in \mathbb{R} .
\end{gathered}
$$

Given an index set $\mathcal{E}$, for a boundary face $F=\cap_{i=1}^{\delta} H_{i}$ for boundary hypersurfaces $H_{i}$, define the subset $\mathcal{E}(F) \subset \mathbb{C}^{p} \times \mathbb{N}^{p}$ by $(z, p)=\left(z_{1}, \ldots, z_{\delta}, p_{1}, \ldots, p_{\delta}\right) \in \mathcal{E}(F)$ if and only if $\left(z_{i}, p_{i}\right) \in \mathcal{E}\left(H_{i}\right)$. We define the Frechet space $\mathcal{A}_{\text {phg }}^{\mathcal{E}}(X)$ as follows. We write $u \in \mathcal{A}_{\text {phg }}^{\mathcal{E}}(X)$ if and only if for each boundary face $F=\cap_{i=1}^{\delta} H_{i}$, writing $\rho_{i}$ for a boundary defining function of $H_{i}, u$ satisfies

$$
\begin{gathered}
u \sim \sum_{(z, p) \in \mathcal{E}(F)} a_{z, p} \rho^{z} \log ^{p} \rho \text { where } \\
\rho^{z}=\prod_{i=1}^{\delta} \rho_{i}^{z_{i}}, \quad \log ^{p} \rho=\prod_{i=1}^{\delta} \log ^{p_{i}} \rho_{i},
\end{gathered}
$$

and the symbol $\sim$ means that

$$
E_{N}=u-\sum_{\substack{(z, p) \in \mathcal{E}(F) \\ \operatorname{Re} z_{i}<N \\ \forall i}} a_{z, p} \rho^{z} \log ^{p} \rho,
$$

where $E_{N}$ is a smooth function on the interior of $X$ which is $O\left(|\rho|^{N}\right)$, where $|\rho|=\left(\rho_{1}^{2}+\cdots+\right.$ $\left.\rho_{\delta}^{2}\right)^{1 / 2}$. Moreover, $E_{N}$ is conormal, meaning that if $\mathcal{V}_{b}=\mathcal{V}_{b}(X)$ denotes the set of smooth vector fields on $X$ that are tangent to all boundary hypersurfaces, then

$$
|\rho|^{-N} \mathcal{V}_{b}^{k} E \subset L^{\infty}
$$

Note that if a phg function $u$ vanishes to infinite order at $H$, then $u$ is polyhomogeneous with index set $\mathcal{E}$ satisfying $\mathcal{E}(H)=\varnothing$.

Lemma 21. Let $X$ denote a mwc, $\mathcal{M}(X)=\left\{H_{i}\right\}_{i \in \mathcal{I}}$ its boundary hypersurfaces, and for each $i \in \mathcal{I}$, let $\rho_{i}$ denote a boundary defining function of $H_{i}$. Given a smooth vector bundle $E \longrightarrow X$, if $\kappa_{i}$ are polyhomogeneous sections on $H_{i}$, then provided

$$
\left.\rho_{i}^{c_{i}} \kappa_{j}\right|_{H_{i} \cap H_{j}}=\left.\rho_{j}^{c_{j}} \kappa_{i}\right|_{H_{i} \cap H_{j}}
$$


there exists a polyhomogeneous conormal distribution $K$ on $X$ satsifying

$$
\left.\rho_{i}^{c_{i}} K\right|_{H_{i}}=\kappa_{i}
$$

Assume moreover that at a particular boundary hypersurface which we take to be $H_{1}$, that we are given an index set $F_{1} \subset \mathbb{C} \times \mathbb{N}$ and polyhomogeneous sections $b_{j, p} \in \mathcal{A}_{\mathrm{phg}}\left(\left.E\right|_{H^{1}} ; H_{1}\right)$. Then given functions $\kappa_{i}$ on $H_{i}, i \neq 1$, there exists a distribution $K$ satisfying A.6 for $i \neq 1$ and such that

$$
K \simeq \sum_{s, p \in F_{1}} \rho_{1}^{s} \log ^{p}\left(\rho_{1}\right) b_{s, p}
$$

provided A.5 holds for $i, j \neq 1$ and furthermore for $i \neq 1$

$$
\left.\kappa_{i} \simeq \rho_{i}^{c_{i}} \sum_{s, p \in F_{1}} \rho_{1}^{s} \log ^{p}\left(\rho_{1}\right) b_{s, p}\right|_{H_{i}}
$$

Remark 22. (1) Note the converse; if $K=\rho_{i}^{-c_{i}} \rho_{j}^{-c_{j}}$ a for some positive function a near $H_{i} \cap H_{j}$ then setting $\left.\rho_{l}^{c_{l}} K\right|_{H_{l}}=\kappa_{l}$ for $l=i, j$, we have $\rho_{j}^{c_{j}} \kappa_{i}=\rho_{i}^{c_{i}} \kappa_{j}$ on $H_{i} \cap H_{j}$.

(2) The matching condition A.5 implies further matching conditions on multifold intersections, e.g. it implies that

$$
\rho_{i}^{c_{i}} \rho_{j}^{c_{j}} \kappa_{l}=\rho_{i}^{c_{i}} \rho_{l}^{c_{l}} \kappa_{j}=\rho_{l}^{c_{l}} \rho_{j}^{c_{j}} \kappa_{i} \text { on } H_{i} \cap H_{j} \cap H_{l} \text {. }
$$

(3) The second matching condition A.8 merely says that the desired data on a bhs $H_{i}$ has the same asymptotic expansion at $H_{1}$ as the the desired distribution restricted to $H_{i}$.

Proof. Denote the number of boundary hypersurfaces of $X$ by $m=|\mathcal{M}|$. There is a number $\delta$ and boundary defining functions $\rho_{i}$ such that the set $\left\{\rho_{i}<\delta\right\}$ is diffeomorphic as mwc's to $H_{i} \times[0, \delta)$. Without loss of generality we take $\delta=1$. Following the remark, for a collection of bhs' $H_{i_{1}}, \ldots, H_{i_{p}}$, the distrubution

$$
\kappa_{i_{1} \ldots i_{p}}=\left.\left(\prod_{i \neq i_{k}} \rho^{c_{i}}\right) \kappa_{i_{k}}\right|_{\rho_{i_{1}}=\cdots=\rho_{i_{p}}=0}
$$

is well-defined independently of the choice of $i_{k} \in\{1, \ldots, m\}$.

Let $\chi(x)$ be a cutoff function with $\chi \equiv 1$ for $x \leq 1 / 3$ and $\chi \equiv 0$ for $x \geq 2 \epsilon / 3$. For the distribution $K$ we may take

$$
K=\sum_{p=1}^{m}(-1)^{p-1} \sum_{1 \leq i_{1}<\cdots<i_{p} \leq m} \kappa_{i_{1} \ldots i_{p}}\left(\prod_{j \in\left\{i_{1}, \ldots, i_{p}\right\}} \chi\left(\rho_{j}\right) \rho_{j}^{-c_{j}}\right) .
$$

For example if $m=2$ then

$$
K=\chi\left(\rho_{1}\right) \rho_{1}^{-c_{1}} \kappa_{1}+\chi\left(\rho_{2}\right) \rho_{2}^{-c_{2}} \kappa_{2}-\chi\left(\rho_{1}\right) \chi\left(\rho_{2}\right) \rho_{1}^{-c_{1}} \rho_{2}^{-c_{2}} \kappa_{12}
$$

Note that each term in the sum defining $K$ defines a polyhomogeneous conormal distribution on all of $X$, as the distribution $\kappa_{i_{1} \ldots i_{p}}$ is defined on a neighborhood of $H_{i_{1}} \cap \cdots \cap H_{i_{p}}$ off which the product $\prod_{j \in\left\{i_{1}, \ldots, i_{p}\right\}} \chi\left(\rho_{j}\right)$ vanishes.

Letting $A_{i_{1} \ldots i_{p}}$ be the term corresponding term in the definition of $K$, note that if $i \notin$ $\left\{i_{1}, \ldots, i_{p}\right\}$ then $\rho_{i}^{c_{i}} A_{i_{1} \ldots i_{p}}=\left.\rho_{i}^{c_{i}} A_{i_{1} \ldots i \ldots i_{p}}\right|_{\rho_{i}=0}$. Fixing $i$, multiplying by $\rho^{c_{i}} K$ and restricting to $\rho_{i}=0$ gives

$$
\begin{aligned}
\left.\rho_{i}^{c_{i}} K\right|_{\rho_{i}=0} & =\kappa_{i}+\left.\sum_{p}^{m-1}(-1)^{p-1} \rho_{i}^{c_{i}}\left(\sum_{\substack{1 \leq i_{1}<\cdots<i_{p} \leq m \\
i \notin\left\{i_{1} \ldots i_{p}\right\}}} A_{i_{1} \ldots i_{p}}-\sum_{\substack{1 \leq i_{1}<\cdots<i_{p+1} \leq m \\
i \in\left\{i_{1} \ldots i_{p+1}\right\}}} A_{i_{1} \ldots i_{p+1}}\right)\right|_{\rho_{i}=0} \\
& =\kappa_{i},
\end{aligned}
$$


which establishes A.6.

We now prove the final statement of the lemma. Let $\chi$ be the cutoff function defined above. First, we claim that under the stated assumptions there exists a distribution $K^{\prime}$ supported in $\left\{\rho_{1} \leq 1\right\}$ satisfying both A.7) (with $K$ replaced by $K^{\prime}$ ) and that

$$
\left.\rho_{i}^{c_{i}} K^{\prime}\right|_{H_{i}}=\chi\left(\rho_{1}\right) \kappa_{i}
$$

for each $i \neq 1$. To see this, take any distribution $K^{\prime \prime}$ supported in $\left\{\rho_{1} \leq 1\right\}$ satisfying (A.7), and note that $a_{i}:=\left.\rho_{i}^{c_{i}} K^{\prime \prime}\right|_{H_{i}}-\chi\left(\rho_{1}\right) \kappa_{i}=O\left(\rho_{1}^{\infty}\right)$. By the support condition, the distribution $K^{\prime}=K^{\prime \prime}-\sum_{i \neq 1} \chi\left(\rho_{i}\right) a_{i}$ is defined globally, has the same asymptotic expansion at $H_{1}$ as $K^{\prime \prime}$, and satisfies A.9. This $K^{\prime}$ will play the role of $\chi\left(\rho_{1}\right) \rho^{-c_{1}} \kappa_{1}$ from the previous paragraph. Concretely, for $1<i_{1}<\cdots<i_{p} \leq m$, let $a_{i_{1} \ldots i_{p}}=\left.\left(\Pi_{j \in\left\{i_{1}, \ldots, i_{p}\right.} \rho_{j}^{c_{j}} K^{\prime}\right)\right|_{H_{i_{1}} \cap \cdots \cap H_{i_{p}}}$. Then we may take

$$
\begin{aligned}
K= & \sum_{p=1}^{m}(-1)^{p-1} \sum_{1<i_{1}<\cdots<i_{p} \leq m} \kappa_{i_{1} \ldots i_{p}}\left(\prod_{j \in\left\{i_{1}, \ldots, i_{p}\right\}} \chi\left(\rho_{j}\right) \rho_{j}^{-c_{j}}\right) \\
& +K^{\prime}+\sum_{p=1}^{m}(-1)^{p-1} \sum_{1<i_{1}<\cdots<i_{p} \leq m} a_{i_{1} \ldots i_{p}}\left(\prod_{j \in\left\{i_{1}, \ldots, i_{p}\right\}} \chi\left(\rho_{j}\right) \rho_{j}^{-c_{j}}\right) .
\end{aligned}
$$

Again, for example if $m=2$ then

$$
K=K^{\prime}+\chi\left(\rho_{2}\right) \rho_{2}^{-c_{2}} \kappa_{2}-\left.\left(\rho_{2}^{c_{1}} K^{\prime}\right)\right|_{H_{2}} \rho_{2}^{-c_{2}} \chi\left(\rho_{2}\right) .
$$

The given expression for $K$ can be directly checked to satisfy A.6 - A.7.

A.1. Melrose's pushforward theorem. Given a map $\beta: X \longrightarrow Y$ between manifolds with corners, if $\mathcal{M}(\bullet)$ with $\bullet=X, Y$ denotes the space of boundary hypersurfaces, then $\beta$ is a b-map if it is smooth and if for each $H \in \mathcal{M}(Y)$ with $\rho_{H}$ a boundary defining function for $H$ then

$$
\beta^{*} \rho_{H}=a \Pi_{H_{j}^{\prime} \in \mathcal{M}(X)} \rho_{H_{1}^{\prime}}^{e\left(H_{1}^{\prime}, H\right)} \rho_{H_{1}^{\prime}}^{e\left(H_{2}^{\prime}, H\right)} \ldots \rho_{H_{1}^{\prime}}^{e\left(H_{N}^{\prime}, H\right)}
$$

where $a \in C^{\infty}(X)$ is non-vanishing and $N$ is the number of boundary hypersurfaces of $Y$ and the $e\left(H^{\prime}, H\right)$ are non-negative integers. This means foremost that $\rho_{H}$ pulls back to a smooth function, and the numbers $e\left(H^{\prime}, H\right)$ simply keep track of the order of vanishing of $\beta^{*} \rho_{H}$ at each face of $X$. The function

$$
e: \mathcal{M}(X) \times \mathcal{M}(Y) \longrightarrow \mathbb{N}_{0}
$$

is the exponent matrix of $\beta$, and $e\left(H^{\prime}, H\right)>0$ means $H^{\prime}$ maps into $H$ via $\beta$.

If a b-map has a few additional properties then it pushes forward polyhomogeneous distributions (more accurately, densities) to polyhomogeneous distributions and their index sets change in a way dictated by the exponent matrix. Note that it follows from the definition of a b-map that every boundary face $F$ of $X$ (meaning an intersection of boundary hypersurfaces), can be associated to a face $\bar{\beta}(F)$ of $Y$ defined to be the unique face with $\beta(x) \in \bar{\beta}(F)^{\circ}$ for every $x \in F^{\circ}$. A b-map $\beta: X \longrightarrow Y$ is a b-fibration if:

- $\beta$ does not increase the codimension of faces, i.e. for each boundary face $F$ of $X$, the associated face $\bar{\beta}(F)$ in $Y$ satisfies that $\operatorname{codim}(F) \leq \operatorname{codim}(\bar{\beta}(F))$.

- Restricted to the interior of any face $F^{\circ}, \beta: F^{\circ} \longrightarrow(\bar{\beta}(F))^{\circ}$ is a fibration of open manifolds in the standard sense.

According to a theorem of Melrose [30] which we state below, a b-fibration pushes forward phg densities to phg densities in a manner we describe now. First, on a manifold with corners we choose a non-vanishing b-density $\mu$, meaning a section of $|\Lambda|^{n}\left({ }^{b} T^{*} X\right)$, the density bundle 
of the b-cotangent bundle. The b-tangent bundle ${ }^{b} T X$ is the bundle whose smooth sections are $\mathcal{V}_{b}$, the vector fields tangent to the boundary. The bundle ${ }^{b} T^{*} X$ is the dual bundle of ${ }^{b} T X$, and near a face $F=\cap_{i=1}^{\delta} H_{i}$ where $\rho_{i}$ are bdf's and $y$ are coordinates on $F$ then, the sections of ${ }^{b} T^{*} X$ take the form

$$
\sum_{i} \xi_{i} \frac{d \rho_{i}}{\rho_{i}}+\eta d y
$$

It follows that near any intersection $F=\cap_{j \in J} H_{j_{q}}$ of boundary hypersurfaces for $J \subset \mathcal{I}$ where $\mathcal{I}$ indexes $\mathcal{M}(X)$ (i.e. any face of $X$ ) that a non-vanishing b-density takes the form

$$
\mu=\left|a \frac{d y \prod_{j \in J} d \rho_{j}}{\prod_{j \in J} \rho_{j}}\right|
$$

for some smooth non-vanishing function $a$ on $X$. A polyhomogeneous b-density $u \in$ $\mathcal{A}_{\text {phg }}^{\mathcal{E}}(X) \otimes|\Lambda|^{n}\left({ }^{b} T^{*} X\right)$ can be written as $f \mu$ for a phg function $f$ and the index set of $u$ is by definition the index set of $f$.

Theorem 23 (Melrose [30]). Let $u \in \mathcal{A}_{\text {phg }}^{\mathcal{E}}(X) \otimes|\Lambda|^{n}\left({ }^{b} T^{*} X\right)$ be a polyhomogeneous b-density on $X$ with index set $\mathcal{E}$, let $f: X \longrightarrow Y$ be a b-fibration with exponent matrix $e$, and define the pushforward $f_{*} u$ to be the distribution on smooth functions $v \in C_{c o m p}^{\infty}(Y)$ acting by $\left\langle f_{*} u, v\right\rangle_{Y}=\left\langle u, f^{*} v\right\rangle_{X}$. Then provided for each $H \in \mathcal{M}(X)$ we have

$$
e\left(H, H^{\prime}\right)=0 \forall H^{\prime} \in \mathcal{M}(Y) \quad \Longrightarrow \quad \mathcal{E}(H)>0,
$$

then $f_{*} u \in \mathcal{A}_{\mathrm{phg}}^{\mathcal{E}^{\prime}}(Y) \otimes|\Lambda|^{n}\left({ }^{b} T^{*} Y\right)$ where

$$
\mathcal{E}^{\prime}(H)=\bar{\bigcup}_{H^{\prime}}\left\{\left(\frac{z}{e\left(H^{\prime}, H\right)}, p\right):(z, p) \in \mathcal{E}(H)\right\},
$$

with the (extended) union taken over $H^{\prime}$ with $e\left(H^{\prime}, H\right)>0$.

The extended union, defined in [31], contains the standard union and possibly more log terms.

A.2. Heat trace asymptotics. We now use Theorem 23 to prove the heat trace formula in Corollary 18 above. The heat trace is equal to

$$
\operatorname{Tr}\left(e^{-t \Delta}\right)=\int_{M} H_{t}(w, w) \mathrm{dVol}_{g}=\sigma_{*}\left(\left(\iota^{*} H_{t}\right) \mathrm{dVol}\right)
$$

where $\iota: M \times[0, \infty) \longrightarrow M \times M \times[0, \infty)$ is the diagonal inclusion and $\sigma: M \times[0, \infty) \longrightarrow$ $[0, \infty)$ is the projection onto the right factor. The natural space here on which to consider $H_{t}$ is $M_{\text {heat }}^{2}$, and thus to evalute this pushforward we must see how $\sigma$ and $\iota$ act on the natural blown up spaces. The following may be easily verified.

(1) The closure $\left(M_{\text {heat }}^{2}\right)_{\Delta}:=\operatorname{cl}\left(\iota\left(M^{\circ} \times(0, \infty)\right)\right)$ is a manifold with corners with 4 boundary hypersurfaces, $\mathrm{sf}, \mathrm{ff}_{1}{ }^{d}, \mathrm{ff}^{d}, \mathrm{tf}^{d}$, equal to the intersection of $\operatorname{cl}\left(\iota\left(M^{\circ} \times(0, \infty)\right)\right)$ with $\mathrm{rf} \cap \mathrm{lf}, \mathrm{ff}_{1}$, ff, and tf, respectively

(2) The map $\sigma$ extends form the interior $M^{\circ} \times(0, \infty)$ to a b-fibration $\sigma:\left(M_{\text {heat }}^{2}\right)_{\Delta} \longrightarrow$ $[0, \infty)$ with exponent matrix

$$
e_{\sigma}(\mathrm{sf})=0, e_{\sigma}\left(\mathrm{ff}_{1}^{d}\right)=2, e_{\sigma}\left(\mathrm{ff}^{d}\right)=2 k, e_{\sigma}\left(\mathrm{tf}^{d}\right)=2 .
$$

To apply the pushforward theorem, we note that the volume density

$$
\mu=\left|\mathrm{dVol}_{g} \frac{d t}{t}\right|=x^{k f+1} \frac{|d x d y d z d t|}{x t}
$$


is equal on $\left(M_{\text {heat }}^{2}\right)_{\Delta}$ to

$$
\mu=a\left(\rho_{\mathrm{sf}} \rho_{\mathrm{ff}_{1} d} \rho_{\mathrm{ff}^{d}}\right)^{k f+1} \mu_{0},
$$

where $\mu_{0}$ is a non-vanishing b-density on $\left(M_{\text {heat }}^{2}\right)_{\Delta}$. Thus $\left(\iota^{*} H\right) \mu$ is phg on $\left(M_{\text {heat }}^{2}\right)_{\Delta}$ with index family $\mathcal{E}^{d}$ satisfying

$$
\begin{gathered}
\inf \mathcal{E}^{d}(\mathrm{sf})=3, \quad \inf \mathcal{E}^{d}\left(\mathrm{ff}_{1}^{d}\right)=-b, \\
\inf \mathcal{E}^{d}\left(\mathrm{ff}^{d}\right)=k(f-n)+1, \quad \mathcal{E}^{d}\left(\mathrm{tf}^{d}\right)=\{-n,-n+1, \ldots\} .
\end{gathered}
$$

Note that $\operatorname{Tr} e^{-t \Delta} \frac{d t}{t}=\sigma_{*}\left(\left(\iota^{*} H_{t}\right) \mu\right)$. The integrability condition A.12 must be checked only for sf and thus holds by Theorem 12 , and we apply the pushforward theorem to obtain that $\operatorname{Tr} e^{-t \Delta}$ is polyhomogeneous with index set

$$
\begin{gathered}
\left\{\left(\zeta_{1} / 2, p_{1}\right):\left(\zeta_{1}, p_{1}\right) \in \mathcal{E}^{d}\left(\mathrm{ff}_{1}{ }^{d}\right)\right\} \bar{U}\left\{\left(\zeta_{2} /(2 k), p_{2}\right):\left(\zeta_{2}, p_{2}\right) \in \mathcal{E}^{d}\left(\mathrm{ff}^{d}\right)\right\} \\
\bar{U}\left\{\left(\zeta_{3} / 2, p_{3}\right):\left(\zeta_{3}, p_{3}\right) \in \mathcal{E}^{d}\left(\mathrm{tf}^{d}\right)\right\} .
\end{gathered}
$$

In particular,

$$
F(t)=\left(\sum_{j=0}^{f} a_{j} t^{-n / 2+j / 2}\right)+c_{0} t^{-(b+1) / 2+1 /(2 k)}+O\left(t^{-(b+1) / 2+1 /(2 k)+\epsilon}\right),
$$

for some $\epsilon>0$. As discussed in [27, Section 3.3], the heat kernel in fact lies in an even calculus and thus the terms for odd $j$ in this sum are equal to 0 , giving the trace formula 4.16). The fact that the leading order term is the volume is standard.

\section{Appendix B. Triple Space}

We will now analyze composition properties for "Volterra" type convolution operators as described in (3.70). To do so, following [31, 15], we construct a "triple space," which we denote by $M_{\text {heat }}^{3}$, which is designed specifically to accomodate the process of composing operators which have the structure of the error terms in (3.71). The structure of our triple space is analogous to that constructed by Grieser and Hunsicker in [15], with slightly different homogeneities and with the added complication that there are time variables involved.

Note that, given $A_{i}, i=1,2$, we want is to make sense of the integral

$$
\int_{M} \int_{0}^{t^{\prime}} A_{1}\left(w, w^{\prime}, t^{\prime}\right) A_{2}\left(w^{\prime}, \widetilde{w}, t-t^{\prime}\right) \operatorname{dVol}_{g}\left(w^{\prime}\right) d t^{\prime}
$$

Define the wedge

$$
W:=\left\{t-t^{\prime} \geq 0\right\} \subset \mathbb{R}_{t}^{+} \times \mathbb{R}_{t^{\prime}}^{+}
$$

and define the left, center, and right projections

$$
\begin{aligned}
\pi_{L}: M \times M \times M \times W & \longrightarrow M \times M \times[0, \infty)_{\mathfrak{t}} \\
\left(w, w^{\prime}, \widetilde{w}, t, t^{\prime}\right) & \longmapsto\left(w, w^{\prime}, t^{\prime}\right) \\
\pi_{C}: M \times M \times M \times W & \longrightarrow M \times M \times[0, \infty)_{\mathfrak{t}} \\
\left(w, w^{\prime}, \widetilde{w}, t, t^{\prime}\right) & \longmapsto(w, \widetilde{w}, t) \\
\pi_{R}: M \times M \times M \times W & \longrightarrow M \times M \times[0, \infty)_{\mathfrak{t}} \\
\left(w, w^{\prime}, \widetilde{w}, t, t^{\prime}\right) & \longmapsto\left(w^{\prime}, \widetilde{w}, t-t^{\prime}\right) .
\end{aligned}
$$

Then, formally, the integral in (B.1) says that the integral kernel of $A_{1} A_{2}$ (as an operator acting by convolution in time) is

$$
\left(A_{1} A_{2}\right)(w, \widetilde{w}, t)=\left(\pi_{C}\right)_{*}\left(\pi_{L}^{*} A_{1}\right)\left(\pi_{R}^{*} A_{2}\right),
$$


where $\left(\pi_{C}\right)_{*}$ denotes the pushforward, i.e. the integral along the fibers of $\pi_{C}$ (which, by the way we have set up the problem, requires the choice of a metric on the fibers which we come to shortly.) Analysis of (B.4) becomes tractable if the space $M^{3} \times W$ is blown up so that the pushforward theorem described in Section A.1 applies.

Note that $M^{3} \times W$ is a manifold with corners with 5 boundary hypersurfaces

$$
\begin{gathered}
\mathrm{L}=\{x=0\}, \quad \mathrm{C}=\left\{x^{\prime}=0\right\}, \quad \mathrm{R}=\{\widetilde{x}=0\} \\
\mathrm{tb}_{1}=\left\{t^{\prime}=0\right\}, \quad \mathrm{tb}_{2}=\left\{t-t^{\prime}=0\right\}
\end{gathered}
$$

It is easy to check that, in the language of Appendix $\mathrm{A}$, the maps $\pi \bullet$ with $\bullet \in\{L, C, R\}$ are b-maps from $M^{3} \times W$ to $M^{2} \times[0, \infty)_{t}$ and the exponent matrices are also easy to compute,

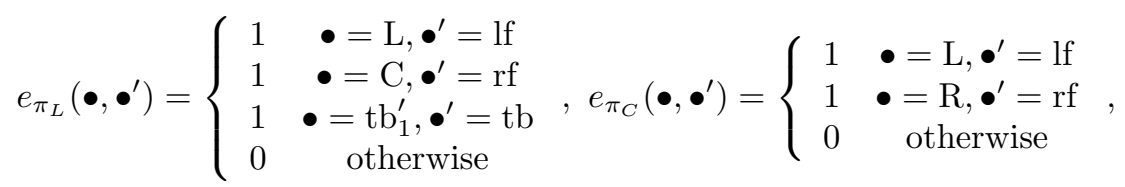

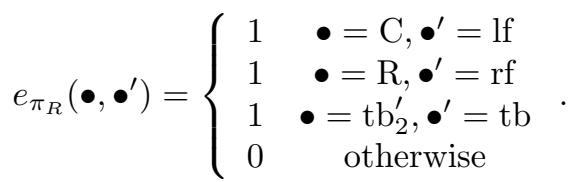

We blow up $M^{3} \times W$ to form a space $\widetilde{\beta}: M_{\text {heat }}^{3} \longrightarrow M^{3} \times W$ in a sequence of steps as follows.

First, consider the three pullbacks of the submanifold $\mathcal{B}_{0}=\{x=\widetilde{x}, y=\widetilde{y}, \widetilde{t}=0\} \subset$ $M^{2} \times[0, \infty)_{\tilde{t}}$ defined in 3.1

$$
\pi_{L}^{-1}\left(\mathcal{B}_{0}\right), \quad \pi_{C}^{-1}\left(\mathcal{B}_{0}\right), \quad \pi_{R}^{-1}\left(\mathcal{B}_{0}\right)
$$

These three sets intersect pair-wise in the triple intersection:

$$
\pi_{L}^{-1}\left(\mathcal{B}_{0}\right) \cap \pi_{C}^{-1}\left(\mathcal{B}_{0}\right)=\pi_{C}^{-1}\left(\mathcal{B}_{0}\right) \cap \pi_{R}^{-1}\left(\mathcal{B}_{0}\right)=\pi_{L}^{-1}\left(\mathcal{B}_{0}\right) \cap \pi_{R}^{-1}\left(\mathcal{B}_{0}\right)=\mathcal{S}
$$

where

$$
\mathcal{S}=\left\{x=x^{\prime}=\widetilde{x}=t=t^{\prime}=y-y^{\prime}=y^{\prime}-\widetilde{y}=0\right\} .
$$

We blow up the set $\mathcal{S}$, with appropriate homogeneities, specifically letting

$$
M_{\text {heat }, 0}^{3}=\left[M^{3} \times W ; \mathcal{S}\right]_{\text {inhom }},
$$

with $t \sim x^{2} \sim\left(x^{\prime}\right)^{2} \sim \widetilde{x}^{2} \sim\left|y-y^{\prime}\right|^{2} \sim\left|y^{\prime}-\widetilde{y}\right|^{2}$, and let $\widetilde{\beta}_{0}: M_{\text {heat }, 0}^{3} \longrightarrow M^{3} \times W$ denote the blowdown map. Call the introduced boundary hypersurface $\mathrm{ff}_{1} \cap$. Near to $\mathrm{ff}_{1} \cap$, we have polar coordinates

$$
\begin{aligned}
\rho_{\cap} & =\left(t+x^{2}+\left(x^{\prime}\right)^{2}+\widetilde{x}^{2}+\left|y-y^{\prime}\right|^{2}+\left|y^{\prime}-\widetilde{y}\right|^{2}\right)^{1 / 2}, \\
\phi^{\cap} & =\left(\frac{t^{\prime}}{\rho_{\cap}^{2}}, \frac{t-t^{\prime}}{\rho_{\cap}^{2}}, \frac{x}{\rho_{\cap}}, \frac{x^{\prime}}{\rho_{\cap}} \frac{\widetilde{x}}{\rho_{\cap}}, \frac{y-y^{\prime}}{\rho_{\cap}}, \frac{y^{\prime}-\widetilde{y}}{\rho_{\cap}}\right) \\
& =:\left(\phi_{t^{\prime}}^{\cap}, \phi_{t-t^{\prime}}^{\cap}, \phi_{x}^{\cap}, \phi_{x^{\prime}}^{\cap}, \phi_{\widetilde{x}}^{\cap}, \phi_{y-y^{\prime}}^{\cap}, \phi_{y^{\prime}-\widetilde{y}}^{\cap}\right) \text { along with } y^{\prime}, z, z^{\prime}, \widetilde{z} .
\end{aligned}
$$

The asymmetry of the $y, y^{\prime}, \widetilde{y}$ in the coordinates is spurious in the sense that if one defines $\phi_{y-\widetilde{y}}^{\cap}=(y-\widetilde{y}) / \rho_{\cap}$, then any two of the $\phi_{y-y^{\prime}}^{\cap}, \phi_{y^{\prime}-\widetilde{y}}^{\cap}$ can be used in $\phi^{\cap}$ by redefining $\rho_{\cap}$ using e.g. $\left|y-y^{\prime}\right|^{2}$ and $|y-\widetilde{y}|^{2}$ (and then using $\phi_{y-y^{\prime}}^{\cap}, \phi_{y^{\prime}-\widetilde{y}}^{\cap}$ ). Either set of coordinates is defined in a colar neighborhood of $\mathrm{ff}_{1} \cap$.

We then blow up the closures of the lifts

$$
\mathcal{S}_{\bullet}:=\operatorname{cl}\left(\left(\pi_{\bullet} \circ \widetilde{\beta}_{0}\right)^{-1}\left(\mathcal{B}_{0}\right) \backslash \mathrm{ff}_{1}^{\cap}\right),
$$


i.e. the rest of the lifts of the $\mathcal{B}_{0}$ via the three projections, where $\bullet \in\{L, C, R\}$. These are disjoint subsets and we blow them up in any order, setting

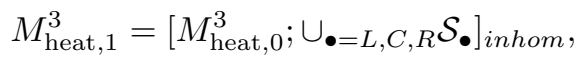

with the appropriate homogeneties, e.g. for $\mathcal{S}_{L}$ we have $t^{\prime} \sim x^{2} \sim\left(x^{\prime}\right)^{2} \sim\left|y-y^{\prime}\right|^{2}$. Again, we have a blowdown map

$$
\widetilde{\beta}_{1}: M_{\text {heat }, 1}^{3} \longrightarrow M \times M \times M \times W .
$$

The new faces we call $\mathrm{ff}_{1}^{\bullet}$ with $\bullet \in\{L, C, R\}$. Coordinates at $\mathrm{ff}_{1}^{L}$ can be determined as follows. Note that $\mathcal{S}_{L}$ is given in the coordinates B.11] by $\phi_{t^{\prime}}^{\bigcap}=\phi_{x}^{\bigcap}=\phi_{x^{\prime}}^{\cap}=\phi_{y-y^{\prime}}^{\bigcap}=0$, and that in a neighborhood of $\mathcal{S}_{L}$ away from $\mathrm{ff}_{1}^{\cap}, \phi_{t^{\prime}}^{\cap} \sim t^{\prime}$. Thus, to match homogeneities with the blowups of the double space, we want to blow this up so that the following give polar coordinates near the intersection of $\mathrm{ff}_{1}^{L}$ and $\mathrm{ff}_{1} \cap$ :

$$
\begin{aligned}
\rho^{L} & =\left(\phi_{t^{\prime}}^{\cap}+\left(\phi^{\cap}\right)_{x}^{2}+\left(\phi^{\cap}\right)_{x^{\prime}}^{2}+\left|\left(\phi^{\cap}\right)_{y-y^{\prime}}\right|^{2}\right)^{1 / 2}, \\
\phi^{L} & =\left(\frac{\phi_{t^{\prime}}^{\cap}}{\left(\rho^{L}\right)^{2}}, \frac{\phi_{x}^{\cap}}{\rho^{L}}, \frac{\phi_{x^{\prime}}^{\cap}}{\rho^{L}}, \frac{\phi_{y-y^{\prime}}^{\cap}}{\rho^{L}}\right) \\
& =:\left(\phi_{t^{\prime}}^{L}, \phi_{x}^{L}, \phi_{x^{\prime}}^{L}, \phi_{y-y^{\prime}}^{L}\right), \text { along with } y^{\prime}, z, z^{\prime}, \widetilde{z}, \rho_{\cap}, \phi_{\widetilde{x}}^{\bigcap}, \phi_{y^{\prime}-\widetilde{y}}^{\cap}, \phi_{t-t^{\prime}}^{\cap}
\end{aligned}
$$

with functions as in B.11). It is also possible to use simpler projective coordinates, as we will see below. Coordinates near $\mathrm{ff}_{1}^{R}$ can be derived similarly by switching $\phi_{t^{\prime}}^{\cap}$ with $\phi_{t-t^{\prime}}^{\cap}$ and $\phi_{x}^{\bigcap}$ with $\phi_{\widetilde{x}}^{\bigcap}$. The situation at $\mathrm{ff}_{1}^{C}$ is slightly different since, writing $\phi_{t}^{\cap}=\phi_{t^{\prime}}^{\bigcap}+\phi_{t-t^{\prime}}^{\bigcap}$, the pullback of $\phi_{t}^{\cap}$ on $M_{\text {heat, } 1}^{2}$ via $\pi_{C}$ vanishes at $\phi_{t^{\prime}}^{\cap}=0=\phi_{t-t^{\prime}}^{\cap}$, and thus $\mathcal{S}_{C}$ is codimension 1 higher than $\mathcal{S}_{\bullet}$ for $\bullet=L, R$.

Here we blow up so that the following give coordinates

$$
\begin{aligned}
\rho^{C} & =\left(\phi_{t}^{\cap}+\left(\phi^{\cap}\right)_{x}^{2}+\left(\phi^{\cap}\right)_{\widetilde{x}}^{2}+\left|\phi_{y-\widetilde{y}}^{\cap}\right|^{2}\right)^{1 / 2}, \\
\left(\phi^{\cap}\right)^{C} & =\left(\frac{\phi_{t^{\prime}}^{\cap}}{\left(\rho^{C}\right)^{2}}, \frac{\phi_{t-t^{\prime}}^{\cap}}{\left.\rho^{C}\right)^{2}}, \frac{\phi_{x}^{\cap}}{\rho^{C}}, \frac{\phi_{\widetilde{x}}^{\cap}}{\rho^{C}}, \frac{\phi_{y-\widetilde{y}}^{\cap}}{\rho^{C}}\right) \\
& =:\left(\phi_{t^{\prime}}^{C}, \phi_{t-t^{\prime}}^{C}, \phi_{x}^{C}, \phi_{\widetilde{x}}^{C}, \phi_{y-\widetilde{y}}^{C}\right), \text { along with } y^{\prime}, z, z^{\prime}, \widetilde{z}, \rho_{\cap}, \phi_{x^{\prime}}^{\cap}, \phi_{y^{\prime}-\widetilde{y}}^{\cap} .
\end{aligned}
$$

Lemma 24. With terminology as in Appendix A.1, the maps $\pi_{\bullet}$ extend from the interior to b-maps

$$
\tilde{\pi}_{\bullet}: M_{\text {heat }, 1}^{3} \longrightarrow M_{\text {heat }, 1}^{2}
$$

for $\bullet \in\{L, C, R\}$ with exponent matrices $e_{\widetilde{\pi}}$ satisfying

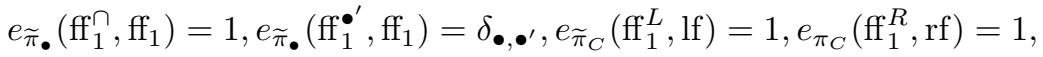

$$
\begin{aligned}
& e_{\widetilde{\pi}_{R}}\left(\mathrm{ff}_{1}^{L}, \mathrm{lf}\right)=1, e_{\widetilde{\pi}_{L}}\left(\mathrm{ff}_{1}^{R}, \mathrm{rf}\right)=1, e_{\widetilde{\pi}_{R}}\left(\mathrm{ff}_{1}^{C}, \mathrm{tb}\right)>0, e_{\widetilde{\pi}_{L}}\left(\mathrm{ff}_{1}^{C}, \mathrm{tb}\right)>0,
\end{aligned}
$$

where $\delta_{\bullet, \bullet^{\prime}}=1$ if $\bullet=\bullet^{\prime}$ and zero otherwise. When $\bullet \in\left\{\mathrm{L}, \mathrm{C}, \mathrm{R}, \mathrm{tb}_{1}^{\prime}, \mathrm{tb}_{2}^{\prime}\right\}$, i.e. when it is the pullback of a boundary hypersurface of $M \times M \times M \times W$ via the blowdown map, then the exponent matrix satisfies (B.5) with $\tilde{\pi}$ replacing $\pi$.

Moreover, $\widetilde{\pi}_{C}$ is a b-fibration in the sense of Appendix A.1.

Remark 25. The significance of the inequalites in (B.17) involving tb is that all the distributions under consideration vanish to infinite order at $\mathrm{tb}$, and thus the pullbacks of these distributions via $\pi_{R}$ will vanish to infinite order at $\mathrm{ff}_{1}^{C}$, and the same for $\pi_{L}$. 
Proof. We verify the lemma for for $\widetilde{\pi}_{C}$ and leave the other nearly identical calculations to the reader. That $\widetilde{\pi}_{C}$ extends to a b-map follows easily by writing the pulling back the coordinates in (3.3) and writing them in terms of those in (B.11). In particular, note that the pullback

$$
\tilde{\pi}_{C}^{*} \rho=\rho_{\cap} \rho^{C},
$$

so the exponent matrix claim holds. The rest of the definitions of b-fibration are easy to check.

Remark 26. The extended map $\widetilde{\pi}_{L}$ is not a b-fibration as it maps the interior of $\mathrm{ff}_{1}^{C}$ to the interior of the face $\mathrm{tb} \cap \mathrm{lf}$ due to the fact that $t=0$ on $W$ implies that $t^{\prime}=0$ also, thus the map increases the codimension of a face. The same holds for $\widetilde{\pi}_{R}$, i.e. $\widetilde{\pi}_{R}\left(\mathrm{ff}_{1}^{C}\right) \subset \mathrm{tb} \cap \mathrm{rf}$.

Next we must blow up the lifts of $\mathcal{B}_{1}$ in (3.4). Since by (B.17), $\tilde{\pi}_{\bullet}$ only maps $\mathrm{ff}_{1}^{\bullet^{\prime}}$ to $\mathrm{ff}_{1}$ if $\bullet=\bullet^{\prime}$, any of the pair-wise intersections is again equal to the triple intersection

$$
\mathcal{S}^{\prime}=\tilde{\pi}_{L}^{-1}\left(\mathcal{B}_{1}\right) \cap \tilde{\pi}_{C}^{-1}\left(\mathcal{B}_{1}\right)=\tilde{\pi}_{C}^{-1}\left(\mathcal{B}_{1}\right) \cap \widetilde{\pi}_{R}^{-1}\left(\mathcal{B}_{1}\right)=\widetilde{\pi}_{L}^{-1}\left(\mathcal{B}_{1}\right) \cap \tilde{\pi}_{R}^{-1}\left(\mathcal{B}_{1}\right) .
$$

Indeed, each is a subset of $\mathrm{ff}_{1}^{\cap}$, and in the polar coordinates defined on the interior of $\mathrm{ff}_{1}^{\cap}$, using the definition of $\mathcal{B}_{1}$ in (3.4)

$$
\mathcal{S}^{\prime}=\left\{\rho=\phi_{t^{\prime}}^{\bigcap}=\phi_{t-t^{\prime}}^{\cap}=0, \phi_{x}^{\cap}=\phi_{x^{\prime}}^{\cap}=\phi_{\widetilde{x}}^{\bigcap}, \phi_{y-y^{\prime}}^{\cap}=\phi_{y-\widetilde{y}}^{\cap}=0\right\},
$$

with no restrictions on $y^{\prime}, z, z^{\prime}, \widetilde{z}$. We form a space $\left[M_{\text {heat }, 1}^{3} ; \mathcal{S}^{\prime}\right]_{\text {inhom }}$ with appropriate homogeneities. To understand this space, note first that near $\mathcal{S}^{\prime}$ we can use projective coordinates on $\mathrm{ff}_{1} \cap$, concretely we can take for example $\widetilde{x}$ to be a boundary defining function of $\mathrm{ff}_{1} \cap$ and coordinates $\widetilde{x}, t^{\prime} / \widetilde{x}^{2},\left(t-t^{\prime}\right) / \widetilde{x}^{2}, x / \widetilde{x}, x^{\prime} / \widetilde{x},\left(y-y^{\prime}\right) / \widetilde{x},\left(y^{\prime}-\widetilde{y}\right) / \widetilde{x}$ to replace the polar coordinates in (B.11). Then the homogeneities are determined by those in the ff blowdown of the double space, and one has coordinates

$$
\begin{aligned}
\bar{\rho}_{\cap} & =\left(\widetilde{x}^{2(k-1)}+\frac{t}{\widetilde{x}^{2}}+\left(\frac{x-\widetilde{x}}{\widetilde{x}}\right)^{2}+\left(\frac{x^{\prime}-\widetilde{x}}{\widetilde{x}}\right)^{2}+\left(\frac{|y-\widetilde{y}|}{\widetilde{x}}\right)^{2}+\left(\frac{\left|y^{\prime}-\widetilde{y}\right|}{\widetilde{x}}\right)^{2}\right)^{1 / 2(k-1)}, \\
\bar{\phi} & :=\left(\bar{\phi}_{\widetilde{x}}, \bar{\phi}_{t^{\prime}}, \bar{\phi}_{t-t^{\prime}}, \bar{\phi}_{x-\widetilde{x}}, \bar{\phi}_{x^{\prime}-\widetilde{x}}, \bar{\phi}_{y-\widetilde{y}}, \bar{\phi}_{y^{\prime}-\widetilde{y}}\right) \\
& =\left(\frac{\widetilde{x}}{\bar{\rho}_{\cap}}, \frac{t^{\prime}}{\widetilde{x}^{2} \bar{\rho}_{\cap}^{2(k-1)}}, \frac{t-t^{\prime}}{\widetilde{x}^{2} \bar{\rho}_{\cap}^{2(k-1)}} \frac{x-\widetilde{x}}{\widetilde{x} \bar{\rho}_{\cap}^{(k-1)}}, \frac{x^{\prime}-\widetilde{x}}{\widetilde{x} \bar{\rho}_{\cap}^{(k-1)}} \frac{y-\widetilde{y}}{\widetilde{x} \bar{\rho}_{\cap}^{(k-1)}}, \frac{y^{\prime}-\widetilde{y}}{\widetilde{x} \bar{\rho}_{\cap}^{(k-1)}}\right) \text { along with } \widetilde{y}, z, z^{\prime}, \widetilde{z} .
\end{aligned}
$$

One can also take coordinates in which $x, x^{\prime}, \widetilde{x}$ are permuted, and the same with $y, y^{\prime}, \widetilde{y}$.

We let $\mathrm{ff}^{\cap}$ denote the introduced boundary hypersurface.

The lifts of the $\widetilde{\pi}_{\bullet}^{-1}\left(\mathcal{B}_{1}\right)$ minus their intersections now have disjoint closures. For example, we have

while

$$
\widetilde{\pi}_{\mathrm{L}}^{-1}\left(\mathcal{B}_{1}\right) \cap \mathrm{ff}_{1}^{\cap} \backslash \mathrm{ff}^{\cap}=\left\{\bar{\phi}_{t^{\prime}}=\bar{\phi}_{x-x^{\prime}}=\bar{\phi}_{y-y^{\prime}}=0\right\}
$$

$$
\widetilde{\pi}_{\mathrm{C}}^{-1}\left(\mathcal{B}_{1}\right) \cap \mathrm{ff}_{1}^{\cap} \backslash \mathrm{ff}^{\cap}=\left\{\bar{\phi}_{t}=\bar{\phi}_{x-\widetilde{x}}=\bar{\phi}_{y-\widetilde{y}}=0\right\},
$$

where $\bar{\phi}_{t}=\bar{\phi}_{t^{\prime}}+\bar{\phi}_{t-t^{\prime}}$ and $\bar{\phi}_{x-\widetilde{x}}=\bar{\phi}_{x-x^{\prime}}+\bar{\phi}_{x^{\prime}-\widetilde{x}}$ and for $\widetilde{\pi}_{\mathrm{R}}$ we have $\bar{\psi}_{x^{\prime}}=\psi_{t-t^{\prime}}=0, \psi_{y^{\prime}}=0$; since $|\bar{\phi}|=1$, these sets are disjoint. Furthermore, the pullbacks satisfy that

$$
\tilde{\pi}_{\bullet}^{-1}\left(\mathcal{B}_{1}\right) \cap \mathrm{ff}_{1}^{\bullet^{\prime}}=\delta_{\bullet, \bullet^{\prime}}
$$


for $\bullet, \bullet^{\prime} \in\{R, C, L\}$, and each intersection is straightforward to write down, e.g. with coordinates as in B.15),

$$
\widetilde{\pi}_{\mathrm{C}}^{-1}\left(\mathcal{B}_{1}\right) \cap \mathrm{ff}_{1}^{C}=\left\{\rho^{C}=\phi_{t^{\prime}}^{C}=\phi_{t-t^{\prime}}^{C}=\phi_{x}^{C}-\phi_{\widetilde{x}}^{C}=0, \phi_{y-\widetilde{y}}^{C}=0\right\} .
$$

We will blow up first the $\widetilde{\pi}_{\bullet}^{-1}\left(\mathcal{B}_{1}\right) \cap \mathrm{ff}_{1}^{\cap}$ and then the $\widetilde{\pi}_{\bullet}^{-1}\left(\mathcal{B}_{1}\right) \cap \mathrm{ff}_{1}^{\bullet}$ with for $\bullet \in\{L, C, R\}$.

In the interior of $\mathrm{ff}_{1}^{\bullet}$ with $\bullet \in\{L, R\}$ the blow ups of the pullbacks of $\mathcal{B}_{1}$ are particularly easy to understand as there we can just pullback the projective coordinates in (3.9) and use these together with the other uneffected coordinates to obtain projective coordinates e.g. near $\pi_{\mathrm{L}} \circ \widetilde{\beta}_{0}^{-1}\left(\mathcal{B}_{1}\right) \cap \mathrm{ff}_{1}^{L}$ valid near the interior of the introduced boundary hypersurface.

$$
x^{\prime}, \quad \sigma=\frac{s-1}{\left(x^{\prime}\right)^{k-1}}=\frac{x-x^{\prime}}{\left(x^{\prime}\right)^{k}}, \quad \eta^{\prime}=\frac{y-y^{\prime}}{\left(x^{\prime}\right)^{k}}, \quad T^{\prime}=\frac{t^{\prime}}{\left(x^{\prime}\right)^{2 k}},
$$

together with $\widetilde{w}, t$ on the introduced boundary hypersurface. In the interior of $\mathrm{ff}_{1}^{C}$, one needs only to remember that the vanishing of the pullback of the $\phi_{t}$ coordinate implies the vanishing of both $\phi_{t^{\prime}}$ and $\phi_{t-t^{\prime}}$. One can use $\widetilde{x}$ as a boundary defining function and then two projective time coordinates $T^{\prime}=t^{\prime} / \widetilde{x}^{2 k}$ and $\widetilde{T}=\left(t-t^{\prime}\right) / \widetilde{x}^{2 k}$. In the interior of $\mathrm{ff}_{1}^{\cap}$ but away from $\mathrm{ff}^{\cap}$, we want the same homogeneities, but now the pullback of $\widetilde{x^{\prime}}$ in the interior of $\mathrm{ff}^{\cap}$ is proportional to $\rho^{\cap}$ and in the interior of the introduced blow up we will have coordinates as in B.21 with all the functions replaced by their $\phi$ couterparts, e.g. $x^{\prime}$ replaced by $\phi_{x^{\prime}}$ and $\frac{y-\tilde{y}}{\left(x^{\prime}\right)^{k}}$ replaced by $\psi_{y-y^{\prime}} / \phi_{x^{\prime}}$.

We focus at the intersection $\mathrm{ff}_{1} \cap \cap \mathrm{fff}_{1}^{\bullet}$, first with $\bullet=C$. Near $\mathcal{S}_{C}$, we can simplify things slightly by using projective coordinates, derived from (B.15) by noting that $\phi_{\widetilde{x}}$ is non-zero at $\mathrm{ff}_{1} \cap \cap \mathrm{ff}_{1} \bullet \cap \mathcal{S}_{C}$ and can thus be used as a boundary defining function. Specifically, take

$$
\widetilde{\mathcal{X}}=\phi_{\widetilde{x}}^{\cap}, \mathcal{X}=\frac{x}{\widetilde{x}}, \mathcal{T}=\frac{t}{\widetilde{x}^{2}}, \mathcal{Y}=\frac{y-\widetilde{y}}{\widetilde{x}},
$$

together with the other (non-polar) coordinates in (B.15). Blowing up to introduce a face $\mathrm{ff}^{\cap, C}$, have

$$
\begin{aligned}
& \mathcal{P}=\left(\mathcal{T}+\rho_{\cap}^{2(k-1)}+(\mathcal{X}-1)^{2}+|\mathcal{Y}|^{2}\right)^{1 /(2(k-1))}, \\
& \Psi=\left(\mathcal{T} / \mathcal{P}^{2(k-1)}, \rho_{\cap} / \mathcal{P},(\mathcal{X}-1) / \mathcal{P}^{k-1}, \mathcal{Y} / \mathcal{P}^{k-1}\right),
\end{aligned}
$$

but it follows that $\mathcal{S}_{1} \cap \mathrm{ff}_{1}{ }^{C}$ intersects $\mathrm{ff}^{\cap, C}$ at $\Psi=(0,1,0,0)$ and thus $\rho^{\cap}$ can be used as a boundary defining function. Again working near $\mathcal{S}_{C}$ we can take $\rho_{\cap}$ as a boundary defining function for $\mathrm{ff}^{\cap, C}$ and use projective coordinates $\rho_{\cap}, \mathcal{T} / \rho_{\cap}^{2(k-1)},(\mathcal{X}-1) / \rho_{\cap}^{k-1}, \mathcal{Y} / \rho_{\cap}^{k-1}$. Using these we blow up $\mathcal{S}_{1} \cap \mathrm{ff}_{1}{ }^{C}$ with

$$
\begin{aligned}
\overline{\mathcal{P}} & =\left(\frac{\mathcal{T}}{\rho_{\cap}^{2(k-1)}}+\left(\phi_{\widetilde{x}}^{\cap}\right)^{2(k-1)}+\frac{(\mathcal{X}-1)^{2}}{\rho_{\cap}^{k-1}}+\frac{|\mathcal{Y}|^{2}}{\rho_{\cap}^{k-1}}\right)^{1 /(2(k-1))}, \\
\bar{\Psi} & =\left(\frac{\mathcal{T}}{\left(\overline{\mathcal{P}} \rho_{\cap}\right)^{2(k-1)}}, \frac{\phi_{\widetilde{x}}^{\cap}}{\overline{\mathcal{P}}}, \frac{\mathcal{X}-1}{\left(\overline{\mathcal{P}} \rho_{\cap}\right)^{k-1}}, \frac{\mathcal{Y}}{\left(\overline{\mathcal{P}} \rho_{\cap}\right)^{k-1}}\right) \\
& =\left(\frac{t}{\widetilde{x}^{2}\left(\overline{\mathcal{P}} \rho_{\cap}\right)^{2(k-1)}}, \frac{\widetilde{x}}{\overline{\mathcal{P}} \rho_{\cap}}, \frac{x-\widetilde{x}}{\widetilde{x}\left(\overline{\mathcal{P}} \rho_{\cap}\right)^{k-1}}, \frac{y-\widetilde{y}}{\widetilde{x}\left(\overline{\mathcal{P}} \rho_{\cap}\right)^{k-1}}\right),
\end{aligned}
$$

and this is the final blow up of $\mathcal{S}_{C}$. The blowups for $\mathcal{S}_{L}, \mathcal{S}_{R}$ are similar and left to the reader. 
Proposition 27 (Incomplete cusp edge heat triple space). The above construction yields a space and blowdown map

$$
\widetilde{\beta}: M_{\text {heat }}^{3} \longrightarrow M \times M \times M \times W
$$

such that the maps $\tilde{\pi}_{\bullet}$ from $(\overline{B .16})$ extend to b-maps $\bar{\pi}_{\bullet}: M_{\text {heat }}^{3} \longrightarrow M_{\text {heat }, 2}^{2}$ with exponent matrix satisfying (B.5), B.17) (with $\pi$ and $\widetilde{\pi}$ replaced by $\bar{\pi}$ ), and

$$
\begin{aligned}
& e_{\bar{\pi} \bullet}\left(\mathrm{ff}^{\cap}, \mathrm{ff}\right)=1, \quad e_{\bar{\pi} \cdot}\left(\mathrm{ff}^{\cap}, \bullet^{\prime}, \mathrm{ff}\right)=e_{\bar{\pi} \bullet}\left(\mathrm{ff}^{\bullet^{\prime}}, \mathrm{ff}\right)=\delta_{\bullet}, \bullet^{\prime} \\
& e_{\bar{\pi}_{L}}\left(\mathrm{ff}^{\cap, R}, \mathrm{ff}_{1}\right)=e_{\bar{\pi}_{R}}\left(\mathrm{ff}^{\cap, L}, \mathrm{ff}_{1}\right)=e_{\bar{\pi}_{C}}\left(\mathrm{ff}^{\cap, R}, \mathrm{ff}_{1}\right)=e_{\bar{\pi}_{C}}\left(\mathrm{ff}^{\cap, L}, \mathrm{ff}_{1}\right)=1 \\
& e_{\bar{\pi}_{L}}\left(\mathrm{ff}^{R}, \mathrm{rf}\right)=e_{\bar{\pi}_{R}}\left(\mathrm{ff}^{L}, \text { lf }\right)=e_{\bar{\pi}_{C}}\left(\mathrm{ff}^{R}, \mathrm{rf}\right)=e_{\bar{\pi}_{C}}\left(\mathrm{ff}^{L}, \text { lf }\right)=1 \\
& e_{\bar{\pi}_{L}}\left(\mathrm{ff}^{\cap, C}, \mathrm{tb}\right), e_{\bar{\pi}_{L}}\left(\mathrm{ff}^{C}, \mathrm{tb}\right), e_{\bar{\pi}_{R}}\left(\mathrm{ff}^{\cap}, C, \mathrm{tb}\right), e_{\bar{\pi}_{R}}\left(\mathrm{ff}^{C}, \mathrm{tb}\right) \geq 1 .
\end{aligned}
$$

Moreover, apart from components of $e_{\bar{\pi}}\left(\mathrm{ff}^{\cap, C}, \bullet^{\prime}\right)$ and $e_{\bar{\pi}_{\bullet}}\left(\mathrm{ff}^{C}, \bullet^{\prime}\right)$ with $\bullet \in\{L, R\}$, all other components are zero.

Again, $\bar{\pi}_{C}$ is a b-fibration.

Proof. Again, we focus on $\bar{\pi}_{C}$. To check that $\widetilde{\pi}_{C}$ extends to a $b-$ map, we pull back the polar coordinates $\bar{\rho}, \bar{\phi}, \widetilde{y}, z, \widetilde{z}$, from (3.7) defined at ff in $M_{\text {heat }, 2}^{2}$. First, we compute

$$
\begin{aligned}
\widetilde{\pi}_{C}^{*} \bar{\rho} & =\widetilde{\pi}_{C}^{*}\left(\left(\left(t / \widetilde{x}^{2}\right)+\widetilde{x}^{2(k-1)}+(s-1)^{2}+(|y-\widetilde{y}| / \widetilde{x})^{2}\right)^{1 / 2(k-1)}\right) \\
& =\left(\mathcal{T}+\widetilde{x}^{2(k-1)}+(\mathcal{X}-1)^{2}+|\mathcal{Y}|^{2}\right)^{1 / 2(k-1)} \\
& =\overline{\mathcal{P}} \rho_{\cap},
\end{aligned}
$$

and then note that

$$
\begin{aligned}
\widetilde{\pi}_{C}^{*} \bar{\phi} & =\widetilde{\pi}_{C}^{*}\left(\frac{t}{\widetilde{x}^{2} \bar{\rho}^{2(k-1)}}, \frac{\widetilde{x}}{\bar{\rho}}, \frac{x-\widetilde{x}}{\widetilde{x} \bar{\rho}^{(k-1)}}, \frac{y-\widetilde{y}}{\widetilde{x} \bar{\rho}^{(k-1)}}\right) \\
& =\left(\frac{t}{\widetilde{x}^{2}\left(\overline{\mathcal{P}} \rho_{\cap}\right)^{2(k-1)}}, \frac{\widetilde{x}}{\overline{\mathcal{P}} \rho_{\cap}}, \frac{x-\widetilde{x}}{\widetilde{x}\left(\overline{\mathcal{P}} \rho_{\cap}\right)^{(k-1)}}, \frac{y-\widetilde{y}}{\widetilde{x}\left(\overline{\mathcal{P}} \rho_{\cap}\right)^{(k-1)}}\right) \\
& =\bar{\Psi} .
\end{aligned}
$$

This establishes both claims for $\bar{\pi}_{C}$. The $R, L$ case are left to the reader.

Proposition 28. For $i=1,2$, let $A_{i} \in \mathcal{A}_{\text {phg }}^{\mathcal{E}_{i}}\left(M_{\text {heat }, 2}^{2}\right)$ with the index sets $\mathcal{E}_{i}$ satisfying $\mathcal{E}_{i}\left(\mathrm{ff}_{1}\right) \geq-3-b-k f, \mathcal{E}_{i}(\mathrm{ff}) \geq-k n-2 k, \mathcal{E}_{i}(\mathrm{lf})=\mathcal{E}_{i}(\mathrm{tb})=\varnothing$ and $\mathcal{E}_{i}(\mathrm{rf})$ satisfying (3.57). Then $A_{3}:=\iint_{0}^{t} A_{1}\left(w, w^{\prime}, t^{\prime}\right) A_{2}\left(w^{\prime}, \widetilde{w}, t-t^{\prime}\right) \mathrm{dVol}_{w^{\prime}} d t^{\prime}$ lies in $\mathcal{A}_{\mathrm{phg}}^{\mathcal{E}_{3}}\left(M_{\text {heat }, 2}^{2}\right)$ where for any $\epsilon>0$,

$$
\begin{aligned}
\inf \mathcal{E}_{3}\left(\mathrm{ff}_{1}\right) & \geq \inf \mathcal{E}_{1}\left(\mathrm{ff}_{1}\right)+\inf \mathcal{E}_{2}\left(\mathrm{ff}_{1}\right)+3+b+k f-\epsilon, \\
\inf \mathcal{E}_{3}(\mathrm{ff}) & \geq \inf \mathcal{E}_{1}(\mathrm{ff})+\inf \mathcal{E}_{2}(\mathrm{ff})+k n+2 k-\epsilon .
\end{aligned}
$$

Remark 29. The constants $k n+2 k$ and $3+b+k f$ in (B.24) should be interpreted, for instance in the case of $\mathrm{ff}$, as saying that the (Volterra type) composition of two operators given by Schwartz kernels as in the theorem has Schwartz kernel whose leading order asymptotic behavior at $\mathrm{ff}$ increases relative to the rate $-k n-2 k$, in particular if both the composed operators grow like $-k n-2 k$ then so does the composition. These are, incidentally, the exact rates of blowup of the heat kernel times $t^{-1}$ at the faces $\mathrm{ff}$ and $\mathrm{ff}_{1}$, and furthermore the fact that the errors $t^{-1} Q$ in (3.71) vanish one order faster than $t^{-1} H$ means, as described above, that taking powers makes them vanish at increasing rates at both $\mathrm{ff}$ and $\mathrm{ff}_{1}$. 
Proof. We write $A_{3}$ as the pushforward of a b-density and then apply the Pushforward Theorem from Section A.1. First we define a non-vanishing b-density $\mu_{0}$ on $M \times M \times M \times W$ as follows. We let $\nu$ be a non-vanishing b-density on $M$ satisfying $\nu=a\left|\frac{d x d y d z}{x}\right|$ for a smooth nonvanishing function $a$ near the boundary, and consider

$$
\mu_{0}=\nu \nu^{\prime} \widetilde{\nu}\left|\frac{d t^{\prime} d t}{t^{\prime}\left(t-t^{\prime}\right)}\right|
$$

where $\nu^{\prime}, \widetilde{\nu}$ are equal to $\nu$ in the primed and tilded coordinates, respectively. Since the blowdown map $\widetilde{\beta}$ from $\mathrm{B} .22$ is a b-map, $\widetilde{\beta}^{*} \mu_{0}$ is a b-density on $M_{\text {heat }}^{3}$, and one checks that

$$
\widetilde{\beta}^{*} \mu_{0}=G \bar{\mu}_{0},
$$

for a non-vanishing b-density $\bar{\mu}_{0}$ on $M_{\text {heat }}^{3}$ and $G \in C^{\infty}\left(M_{\text {heat }}^{3}\right)$ satisfying that for some non-vanishing smooth function $G^{\prime}$,

$$
\begin{aligned}
G=G^{\prime} & \left(\rho_{\mathrm{ff}_{1} L} \rho_{\mathrm{ff}_{1} C} \rho_{\mathrm{ff}_{1} R}\right)^{b} \rho_{\mathrm{ff}_{1} \cap}^{2 b}\left(\rho_{\mathrm{ff}^{L}} \rho_{\mathrm{ff}^{C}} \rho_{\mathrm{ff}^{R}}\right)^{k b+k-1} \\
& \times\left(\rho_{\mathrm{ff} \cap, L} \rho_{\mathrm{ff} \cap, C} \rho_{\mathrm{ff} \cap, R}\right)^{(k+1) b+k-1} \rho_{\mathrm{ff} \cap}^{2 k b+2(k-1)} .
\end{aligned}
$$

Then we can write the desired pushforward as a pushforward of a b-density, specifically

$$
\begin{aligned}
A_{3}\left(\nu \widetilde{\nu}\left|\frac{d t}{t}\right|\right) & =\left(\pi_{C}\right)_{*}\left(\pi_{L}^{*} A_{1} \pi_{R}^{*} A_{2} \cdot\left(\left(t^{\prime} / t\right)\left(t-t^{\prime}\right)\right) F\left(w^{\prime}\right) \mu_{0}\right) \\
& =\left(\bar{\pi}_{C}\right)_{*}\left(\bar{\pi}_{L}^{*} A_{1} \bar{\pi}_{R}^{*} A_{2} \cdot \widetilde{\beta}^{*}\left(\left(t^{\prime} / t\right)\left(t-t^{\prime}\right) F\left(w^{\prime}\right) \mu_{0}\right)\right)
\end{aligned}
$$

where $F$ is the function defined by $\mathrm{dVol}_{g}=F \nu$ and in particular

$$
F=a x^{k f+1}
$$

where $a$ is a non-vanishing polyhomogeneous function on $M$, and $\nu, \widetilde{\nu}$ are the pullbacks of the density $\nu$ above to the left and right spacial factors of $M \times M \times \mathbb{R}^{+}$. To find the asymptotics of $A_{3}$ itself we must compute the asymptotics of the densities on the left hand side of B.26); Letting $\beta_{2}$ again denote the blowdown map $M_{\text {heat }, 2}^{2} \longrightarrow M \times M \times[0, \infty)$ in (3.8), we check that

$$
\beta_{2}^{*}\left(\left(\pi_{L}^{\prime}\right)^{*} \nu\left(\pi_{R}^{\prime}\right)^{*} \nu \frac{d t}{t}\right)=\rho_{\mathrm{ff}}^{b} \rho_{\mathrm{ff}}^{b k+k-1} \mu_{2}
$$

where $\mu_{2}$ is a non-vanishing b-density on $M_{\text {heat, } 2}^{2}$. Thus from (B.26), if the distribution $\left(\bar{\pi}_{C}\right)_{*}\left(\bar{\pi}_{L}^{*} A_{1} \bar{\pi}_{R}^{*} A_{2} \cdot \widetilde{\beta}^{*}\left(\left(t^{\prime} / t\right)\left(t-t^{\prime}\right) F\left(w^{\prime}\right) \mu_{0}\right)\right)$ is polyhomogeneous with index set $\mathcal{E}_{3}^{\prime}$ then $A_{3}$ is phg with index set $\mathcal{E}_{3}$ satisfying

$$
\mathcal{E}_{3}\left(\mathrm{ff}_{1}\right)=\mathcal{E}_{3}^{\prime}\left(\mathrm{ff}_{1}\right)-b, \quad \mathcal{E}_{3}(\mathrm{ff})=\mathcal{E}_{3}^{\prime}(\mathrm{ff})-(k b+k-1),
$$

and $\mathcal{E}_{3}(\bullet)=\mathcal{E}_{3}^{\prime}(\bullet)$ otherwise.

Thus the index family of $\bar{\pi}_{L}^{*} A_{1} \bar{\pi}_{R}^{*} A_{2} \cdot \widetilde{\beta}^{*}\left(\left(t^{\prime} / t\right)\left(t-t^{\prime}\right) F\left(w^{\prime}\right)\right)$ must be determined. To determine $\bar{\pi}_{L}^{*} A_{1}$, we see that, at a bhs $H$ of $M_{\text {heat }}^{3}$, the index set of $\bar{\pi}_{L}^{*} A_{1}$ is simply the index set of $A_{1}$ at the bhs $H^{\prime}$ of $M_{\text {heat, } 2}^{2}$ at which $H$ is incident. Thus from our work above 
we see that $\bar{\pi}_{L}^{*} A_{1}$ has index set $\overline{\mathcal{E}}_{1}$ satisfying

$$
\begin{aligned}
\overline{\mathcal{E}}_{1}(\mathrm{~L}) & =\mathcal{E}_{1}(\mathrm{lf})=\varnothing \\
\overline{\mathcal{E}}_{1}(\mathrm{C})=\overline{\mathcal{E}}_{1}\left(\mathrm{ff}_{1}^{R}\right)=\overline{\mathcal{E}}_{1}\left(\mathrm{ff}^{R}\right) & =\mathcal{E}_{1}(\mathrm{rf}) \\
\overline{\mathcal{E}}_{1}\left(\mathrm{tb}_{1}^{\prime}\right)=\overline{\mathcal{E}}_{1}\left(\mathrm{ff}_{1}{ }^{C}\right)=\overline{\mathcal{E}}_{1}\left(\mathrm{ff}^{\cap, C}\right)=\overline{\mathcal{E}}_{1}\left(\mathrm{ff}^{C}\right) & =\mathcal{E}_{1}(\mathrm{tb})=\varnothing \\
\overline{\mathcal{E}}_{1}\left(\mathrm{ff}_{1}^{\cap}\right)=\overline{\mathcal{E}}_{1}\left(\mathrm{ff}_{1}{ }^{L}\right)=\overline{\mathcal{E}}_{1}\left(\mathrm{ff}^{\cap, R}\right) & =\mathcal{E}_{1}\left(\mathrm{ff}_{1}\right) \\
\overline{\mathcal{E}}_{1}\left(\mathrm{ff}^{\cap}\right)=\overline{\mathcal{E}}_{1}\left(\mathrm{ff}^{\cap, L}\right)=\overline{\mathcal{E}}_{1}\left(\mathrm{ff}^{L}\right) & =\mathcal{E}_{1}(\mathrm{ff}) \\
\overline{\mathcal{E}}_{1}(\mathrm{R}) & =\mathbb{Z},
\end{aligned}
$$

the last line coming from the fact that $\bar{\pi}_{L}^{*} A_{1}$ is independent of $\widetilde{x}$, in particular is smooth up to R. The index set $\overline{\mathcal{E}}_{2}$ of $\bar{\pi}_{R}^{*} A_{2}$ has the same expression in terms of $\mathcal{E}_{2}$ but with all 'R's switched with 'L's, all lf's with rf's, and all 1's with 2's (except of course for the 1 in the subscript of $\left.\mathrm{ff}_{1}\right)$. For example, (c.f. the second line above) $\overline{\mathcal{E}}_{2}(\mathrm{C}), \overline{\mathcal{E}}_{2}\left(\mathrm{ff}_{1}{ }^{L}\right), \overline{\mathcal{E}}_{2}\left(\mathrm{ff}^{L}\right)$ are all equal to $\mathcal{E}_{2}$ (lf), which is assumed to be $\varnothing$. If we define the operation $\mathcal{E}_{1} \oplus \mathcal{E}_{2}$ on index sets to denote the index set whose elements are sums of elements of the two index sets, It follows that $\bar{\pi}_{L}^{*} A_{1} \bar{\pi}_{R}^{*} A_{2}$ is polyhomogeneous with index set $\mathcal{F}$ satisfying

$$
\begin{array}{r}
\left\{\mathcal{F}(\mathrm{C}), \mathcal{F}(\mathrm{L}), \mathcal{F}\left(\mathrm{ff}_{1}{ }^{L}\right), \mathcal{F}\left(\mathrm{ff}^{L}\right), \mathcal{F}\left(\mathrm{tb}_{1}^{\prime}\right), \mathcal{F}\left(\mathrm{tb}_{2}^{\prime}\right), \mathcal{F}\left(\mathrm{ff}_{1}^{C}\right), \mathcal{F}\left(\mathrm{ff}^{\cap}, C\right), \mathcal{F}\left(\mathrm{ff}^{C}\right)\right\}=\varnothing \\
\mathcal{F}\left(\mathrm{ff}_{1}^{\cap}\right)=\mathcal{E}_{1}\left(\mathrm{ff}_{1}\right) \oplus \mathcal{E}_{2}\left(\mathrm{ff}_{1}\right), \quad \mathcal{F}\left(\mathrm{ff}^{\cap}\right)=\mathcal{E}_{1}(\mathrm{ff}) \oplus \mathcal{E}_{2}(\mathrm{ff}), \quad \mathcal{F}(\mathrm{R})=\mathcal{E}_{2}(\mathrm{rf}) \\
\mathcal{F}\left(\mathrm{ff}_{1}^{R}\right)=\mathcal{E}_{1}(\mathrm{rf}) \oplus \mathcal{E}_{2}\left(\mathrm{ff}_{1}\right), \quad \mathcal{F}\left(\mathrm{ff}^{R}\right)=\mathcal{E}_{1}(\mathrm{rf}) \oplus \mathcal{E}_{2}(\mathrm{ff}) \\
\mathcal{F}\left(\mathrm{ff}^{\cap}, R\right)=\mathcal{E}_{1}\left(\mathrm{ff}_{1}\right) \oplus \mathcal{E}_{2}(\mathrm{ff}), \quad \mathcal{F}\left(\mathrm{ff}^{\cap, L}\right)=\mathcal{E}_{1}(\mathrm{ff}) \oplus \mathcal{E}_{2}\left(\mathrm{ff}_{1}\right) .
\end{array}
$$

Now we compute the asymptotics of the term $\widetilde{\beta}^{*}\left(\left(\left(t^{\prime} / t\right)\left(t-t^{\prime}\right)\right) F\left(w^{\prime}\right) \mu_{0}\right)=\widetilde{\beta}^{*}\left(\left(\left(t^{\prime} / t\right)(t-\right.\right.$ $\left.\left.\left.t^{\prime}\right)\right) F\left(w^{\prime}\right)\right) G \bar{\mu}_{0}$ with $G$ in $\left(\mathrm{B} .25\right.$. First, write $\widetilde{\beta}^{*}\left(\left(t\left(t-t^{\prime}\right)\right) F\left(w^{\prime}\right)\right)=\bar{\pi}_{L}^{*}(\mathfrak{t}) \bar{\pi}_{R}^{*}(\mathfrak{t}) \bar{\pi}_{R}^{*}(F)$ where $F$ is thought of as a function of the left factor of $M \times M \times[0, \infty)$. Recalling $\rho, \bar{\rho}$ from $(3.3)$ and (3.7), respectively, and letting a denote a polyhomogeneous function which is smooth and non-vanishing up to boundary hypersurfaces $\bullet$ for which $\mathcal{F}(\bullet) \neq \varnothing$ (and whose value will change from line to line), we compute

$$
\begin{aligned}
& \widetilde{\beta}^{*}\left(\frac{t^{\prime}\left(t-t^{\prime}\right)}{t} F\left(w^{\prime}\right)\right)=a \frac{\bar{\pi}_{L}^{*}\left(\rho^{2} \bar{\rho}^{2 k}\right) \bar{\pi}_{R}^{*}\left(\rho^{2} \bar{\rho}^{2 k}\right)}{\bar{\pi}_{C}^{*}\left(\rho^{2} \bar{\rho}^{2 k}\right)} \bar{\pi}_{R}^{*}\left(\left(\rho_{\mathrm{lf}} \rho \bar{\rho}\right)^{k f+1}\right)
\end{aligned}
$$

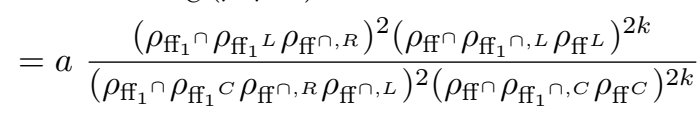

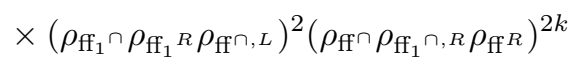

$$
\begin{aligned}
& \times\left(\rho_{C} \rho_{\mathrm{ff}_{1} L} \rho_{\mathrm{fff}} L \rho_{\mathrm{ff}_{1}} \cap \rho_{\mathrm{ff}_{1} R} \rho_{\mathrm{ff} \cap, L} \rho_{\mathrm{ff}} \cap \rho_{\mathrm{ff} \cap, R} \rho_{\mathrm{ff}}\right)^{k f+1}
\end{aligned}
$$

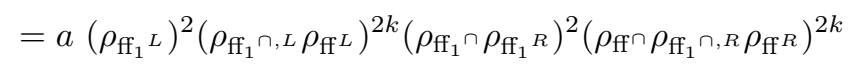

$$
\begin{aligned}
& \times\left(\rho_{C} \rho_{\mathrm{ff}_{1} L} \rho_{\mathrm{ff}} L \rho_{\mathrm{ff}_{1} \cap} \rho_{\mathrm{ff}_{1} R} \rho_{\mathrm{ff} \cap, L} \rho_{\mathrm{ff}} \cap \rho_{\mathrm{ff} \cap, R} \rho_{\mathrm{ff}}\right)^{k f+1}
\end{aligned}
$$

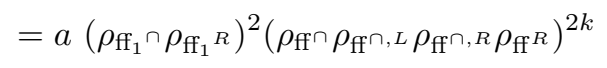

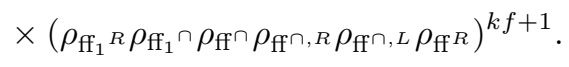


Putting this all together, we see that $\bar{\pi}_{L}^{*} A_{1} \bar{\pi}_{R}^{*} A_{2} \cdot \widetilde{\beta}^{*}\left(\left(\left(t^{\prime} / t\right)\left(t-t^{\prime}\right)\right) F\left(w^{\prime}\right) \mu_{0}\right)$ is polyhomogeneous with index set $\overline{\mathcal{F}}$

$$
\begin{aligned}
\overline{\mathcal{F}}\left(\mathrm{ff}_{1}^{\cap}\right) & =\mathcal{E}_{1}\left(\mathrm{ff}_{1}\right) \oplus \mathcal{E}_{2}\left(\mathrm{ff}_{1}\right)+(3+k f+2 b), \\
\overline{\mathcal{F}}\left(\mathrm{ff}^{\cap}\right) & =\mathcal{E}_{1}(\mathrm{ff}) \oplus \mathcal{E}_{2}(\mathrm{ff})+(1+2 k+k f+2 k b), \\
\overline{\mathcal{F}}(\mathrm{R}) & =\mathcal{E}_{2}(\mathrm{rf}) \\
\overline{\mathcal{F}}\left(\mathrm{ff}_{1}^{R}\right) & =\mathcal{E}_{1}(\mathrm{rf}) \oplus \mathcal{E}_{2}\left(\mathrm{ff}_{1}\right)+(3+k f+b), \\
\overline{\mathcal{F}}\left(\mathrm{ff}^{R}\right) & =\mathcal{E}_{1}(\mathrm{rf}) \oplus \mathcal{E}_{2}(\mathrm{ff})+(1+2 k+k f+k b) \\
\overline{\mathcal{F}}\left(\mathrm{ff}^{\cap}, R\right. & =\mathcal{E}_{1}\left(\mathrm{ff}_{1}\right) \oplus \mathcal{E}_{2}(\mathrm{ff})+(1+2 k+k f+(k+1) b), \\
\overline{\mathcal{F}}\left(\mathrm{ff}^{\cap, L}\right) & =\mathcal{E}_{1}(\mathrm{ff}) \oplus \mathcal{E}_{2}\left(\mathrm{ff}_{1}\right)+(1+2 k+k f+(k+1) b),
\end{aligned}
$$

and $\overline{\mathcal{F}}(\bullet)=\varnothing$ for all other values of $\bullet$.

Now we apply Theorem 23 to analyze $\left(\bar{\pi}_{C}\right)_{*}\left(\pi_{L}^{*} A_{1} \pi_{R}^{*} A_{2} \cdot\left(\left(t^{\prime} / t\right)\left(t-t^{\prime}\right)\right) F\left(w^{\prime}\right) \mu_{0}\right)$ from (B.26). To check that the conditions of the theorem hold, we first recall that $\bar{\pi}_{C}$ is a b-fibration. Also, note that $e_{\bar{\pi}_{C}}\left(\mathrm{C}, H^{\prime}\right)=e_{\bar{\pi}_{C}}\left(\mathrm{tb}_{1}^{\prime}, H^{\prime}\right)=e_{\bar{\pi}_{C}}\left(\mathrm{tb}_{2}^{\prime}, H^{\prime}\right)=0$ for all $H^{\prime} \in \mathcal{M}\left(M_{\text {heat, } 2}^{2}\right)$, and so we must check the inte grability condition there, but by below B.29 we have $\overline{\mathcal{F}}(\mathrm{C})=\overline{\mathcal{F}}\left(\mathrm{tb}_{1}^{\prime}\right)=\overline{\mathcal{F}}\left(\mathrm{tb}_{2}^{\prime}\right)=\varnothing$, so the integrability condition holds. Thus $A_{3}\left(\pi_{L}^{\prime}\right)^{*} \nu\left(\pi_{R}^{\prime}\right)^{*} \nu$ is phg on $M_{\text {heat }, 2}^{2}$ with index set $\mathcal{E}_{3}^{\prime}$ satisfying

$$
\begin{aligned}
& \mathcal{E}_{3}^{\prime}(\text { lf })=\overline{\mathcal{F}}(\mathrm{L}) \overline{\mathcal{F}}\left(\mathrm{ff}_{1}{ }^{L}\right) \cup \overline{\mathcal{F}}\left(\mathrm{ff}^{L}\right)=\varnothing \\
& \mathcal{E}_{3}^{\prime}(\mathrm{rf})=\overline{\mathcal{F}}(\mathrm{R}) \varpi \overline{\mathcal{F}}\left(\mathrm{ff}_{1}{ }^{R}\right) \cup \overline{\mathcal{F}}\left(\mathrm{ff}^{R}\right)
\end{aligned}
$$

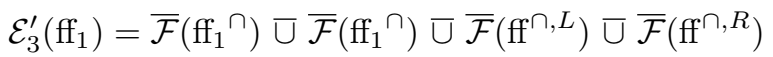

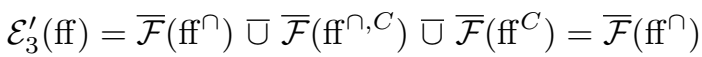

$$
\begin{aligned}
& \mathcal{E}_{3}^{\prime}(\mathrm{tb})=\varnothing \text {, }
\end{aligned}
$$

where we used from below $($ B.29 that various bhs's have infinite order vanishing. From this we see that the bounds in Proposition 28 hold, in particular that for any $\epsilon>0$,

$$
\begin{array}{r}
\inf \mathcal{E}_{3}^{\prime}\left(\mathrm{ff}_{1}\right) \geq \inf \mathcal{E}_{1}\left(\mathrm{ff}_{1}\right)+\inf \mathcal{E}_{2}\left(\mathrm{ff}_{1}\right)+3+k f+2 b-\epsilon, \\
\mathcal{E}_{3}^{\prime}(\mathrm{ff})=\inf \mathcal{E}_{1}(\mathrm{ff})+\inf \mathcal{E}_{2}(\mathrm{ff})+1+k+k n-\epsilon,
\end{array}
$$

and thus by (B.27) the acutal index set $\mathcal{E}_{3}$ of $A_{3}$ satisfies (B.24), and the proof is complete.

\section{REFERENCES}

[1] M. Abramowitz and I. A. Stegun. Handbook of mathematical functions with formulas, graphs, and mathematical tables, volume 55 of National Bureau of Standards Applied Mathematics Series. For sale by the Superintendent of Documents, U.S. Government Printing Office, Washington, D.C., 1964.

[2] P. Albin, É. Leichtnam, R. Mazzeo, and P. Piazza. The signature package on Witt spaces. Ann. Sci. Éc. Norm. Supér. (4), 45(2):241-310, 2012.

[3] P. Albin, É. Leichtnam, R. Mazzeo, and P. Piazza. Hodge theory on Cheeger spaces. 2013. Available online.

[4] P. Albin and F. Rochon. Families index for manifolds with hyperbolic cusp singularities. Int. Math. Res. Not. IMRN, (4):625-697, 2009.

[5] N. Berline, E. Getzler, and M. Vergne. Heat kernels and Dirac operators. Grundlehren Text Editions. Springer-Verlag, Berlin, 2004. Corrected reprint of the 1992 original.

[6] J. Brüning and R. Seeley. The expansion of the resolvent near a singular stratum of conical type. $J$. Funct. Anal., 95(2):255-290, 1991. 
[7] J. Cheeger. On the spectral geometry of spaces with cone-like singularities. Proc. Nat. Acad. Sci. U.S.A., 76(5):2103-2106, 1979.

[8] J. Cheeger. On the Hodge theory of Riemannian pseudomanifolds. In Geometry of the Laplace operator (Proc. Sympos. Pure Math., Univ. Hawaii, Honolulu, Hawaii, 1979), Proc. Sympos. Pure Math., XXXVI, pages 91-146. Amer. Math. Soc., Providence, R.I., 1980.

[9] J. Cheeger. Spectral geometry of singular Riemannian spaces. J. Differential Geom., 18(4):575-657 (1984), 1983.

[10] C. Debord, J.-M. Lescure, and F. Rochon. Pseudodifferential operators on manifolds with fibred corners. available online at arXiv:1112.4575 2011.

[11] J. B. Gil, T. Krainer, and G. A. Mendoza. On the closure of elliptic wedge operators. In Microlocal methods in mathematical physics and global analysis, Trends Math., pages 55-58. Birkhäuser/Springer, Basel, 2013.

[12] M. Goresky and R. MacPherson. Intersection homology theory. Topology, 19(2):135-162, 1980.

[13] M. Goresky and R. MacPherson. Intersection homology. II. Invent. Math., 72(1):77-129, 1983.

[14] D. Grieser. Notes on heat kernel asymptotics. Available online, 2004. http://www.staff.unioldenburg.de/daniel.grieser/wwwlehre/Schriebe/heat.pdf

[15] D. Grieser and E. Hunsicker. Pseudodidfferential operator calculus for generalized $\mathbb{Q}$-rank 1 locally symmetric spaces, i. J. Funct. Anal., 257:3748-3801, 2009.

[16] J. Harris and I. Morrison. Moduli of curves, volume 187 of Graduate Texts in Mathematics. SpringerVerlag, New York, 1998.

[17] T. Hausel, E. Hunsicker, and R. Mazzeo. Hodge cohomology of gravitational instantons. Duke Math. J., 122(3):485-548, 2004.

[18] L. Hörmander. The analysis of linear partial differential operators. I. Classics in Mathematics. SpringerVerlag, Berlin, 1983.

[19] E. Hunsicker and R. Rochon. Hodge cohomology of iterated fibred cusp metrics on witt spaces. available online at arXiv:1206.0984, 2012.

[20] L. Ji, R. Mazzeo, W. Müller, and A. Vasy. Spectral theory for the weil-petersson laplacian on the riemann moduli space. Comment. Math. Helv., 89(4):867-894, 2014.

[21] C. Kottke and R. Melrose. Generalized blow-up of corners and fiber products. In Microlocal methods in mathematical physics and global analysis, Trends Math., pages 59-62. Birkhäuser/Springer, Basel, 2013.

[22] M. Lesch. Operators of Fuchs type, conical singularities, and asymptotic methods, volume 136 of Teubner-Texte sur Math. B.G. Teubner, Stuttgart, Leipzig, 1997.

[23] K. Liu, X. Sun, and S.-T. Yau. New results on the geometry of the moduli space of Riemann surfaces. Sci. China Ser. A, 51(4):632-651, 2008.

[24] K. Liu, X. Sun, and S.-T. Yau. Goodness of canonical metrics on the moduli space of Riemann surfaces. Pure Appl. Math. Q., 10(2):223-243, 2014.

[25] R. Mazzeo. Elliptic theory of differential edge operators. I. Comm. Partial Differential Equations, 16(10):1615-1664, 1991.

[26] R. Mazzeo and J. Swoboda. Asymptotics of the Weil-Petersson metric. available online at arXiv:1503.02365, 2015.

[27] R. Mazzeo and B. Vertman. Analytic torsion on manifolds with edges. Adv. Math., 231(2):1000-1040, 2012.

[28] R. Melrose and X. Zhu. forthcoming work. 2015.

[29] R. B. Melrose. Differential Analysis on Manifolds with Corners. Available online.

[30] R. B. Melrose. Calculus of conormal distributions on manifolds with corners. Int Math Res Notices, 1992:51-61, 1992.

[31] R. B. Melrose. The Atiyah-Patodi-Singer index theorem, volume 4 of Research Notes in Mathematics. A K Peters Ltd., Wellesley, MA, 1993.

[32] E. A. Mooers. Heat kernel asymptotics on manifolds with conic singularities. J. Anal. Math., 78:1-36, 1999.

[33] M. Reed and B. Simon. Methods of modern mathematical physics. I. Academic Press Inc. [Harcourt Brace Jovanovich Publishers], New York, second edition, 1980. Functional analysis.

[34] B.-W. Schulze. Pseudo-differential calculus on manifolds with geometric singularities. In Pseudodifferential operators: partial differential equations and time-frequency analysis, volume 52 of Fields Inst. Commun., pages 37-83. Amer. Math. Soc., Providence, RI, 2007. 
[35] M. E. Taylor. Partial differential equations II. Qualitative studies of linear equations, volume 116 of Applied Mathematical Sciences. Springer, New York, second edition, 2011.

[36] S. Wolpert. On the Weil-Petersson geometry of the moduli space of curves. Amer. J. Math., 107(4):969997, 1985.

[37] S. Yamada. On the geometry of Weil-Petersson completion of Teichmüller spaces. Math. Res. Lett., 11(2-3):327-344, 2004.

[38] S. Zucker. $L_{2}$ cohomology of warped products and arithmetic groups. Invent. Math., 70(2):169-218, $1982 / 83$.

Department of Mathematics, Johns Hopkins University

E-mail address: jgell@math.jhu.edu

Mathematisches InStitut DER LMU MÜNCheN

E-mail address: swoboda@math.1mu.de 